\title{
The Cost of Fault Tolerance in Multi-Party Communication Complexity*
}

\author{
Binbin Chen \\ Advanced Digital Sciences Center \\ Singapore \\ binbin.chen@adsc.com.sg \\ Yuda Zhao \\ National University of Singapore \\ Singapore \\ zhaoyuda@comp.nus.edu.sg
}

\author{
Haifeng $\mathrm{Yu}$ \\ National University of Singapore \\ Singapore \\ haifeng@comp.nus.edu.sg \\ Phillip B. Gibbons \\ Intel Labs \\ Pittsburgh, PA, USA \\ phillip.b.gibbons@intel.com
}

May 2012

\begin{abstract}
Multi-party communication complexity involves distributed computation of a function over inputs held by multiple distributed players. A key focus of distributed computing research, since the very beginning, has been to tolerate crash failures. It is thus natural to ask "If we want to compute a certain function in a fault-tolerant way, what will the communication complexity be?" This natural question, interestingly, has not been formally posed and thoroughly studied prior to this work.

Whether fault-tolerant communication complexity is interesting to study largely depends on how big a difference failures make. This paper proves that the impact of failures is significant, at least for the SUM aggregation function in general networks: As our central contribution, we prove that there exists (at least) an exponential gap between the non-fault-tolerant and fault-tolerant communication complexity of SUM. Our results also imply the optimality (within polylog factors) of some recent fault-tolerant protocols for computing SUM via duplicate-insensitive techniques, thereby answering an open question as well.

Part of our results are obtained via a novel reduction from a new two-party problem UNIONSIZECP that we introduce. UNIONSIZECP comes with a novel cycle promise, which is the key enabler of our reduction. We further prove that this cycle promise and UNIONSIZECP likely play a fundamental role in reasoning about fault-tolerant communication complexity.
\end{abstract}

${ }^{*}$ This article is the full Technical Report version of the PODC'12 paper with the same title. The first three authors of this article are alphabetically ordered. 


\section{Introduction}

Fault tolerance in communication complexity and our exponential gap. Multi-party communication complexity [10] involves distributed computation of a function over inputs held by multiple distributed players. A key focus of distributed computing research, since the very beginning, has been to tolerate failures. (Throughout this paper, failures refer to node crash failures unless otherwise mentioned.) It is thus natural to ask "If we want to compute a certain function in a fault-tolerant way, what will the communication complexity be?" For the question to be meaningful, we impose two restrictions before moving forward. First, we allow the computation to ignore/omit the inputs held by those players that have failed (i.e., crashed) or been disconnected. This means that the function needs to be well-defined over any subset of the inputs. Second, we will assume that there is one special root player that never fails and only this player needs to learn the final result. This allows us to focus on the communication complexity of the function instead of the difficulty of, for example, achieving fault-tolerant distributed consensus. This also nicely maps to our target scenarios later in wireless sensor networks and wireless ad-hoc networks, where the root corresponds to the base station.

While the above question is natural, interestingly, it has not been formally posed and thoroughly studied - see later for our speculations on the possible reasons. Whether such fault-tolerant communication complexity is interesting to study, given the extensive research on "non-fault-tolerant" communication complexity, largely depends on how big a difference failures can make. This paper proves that the impact of failures is significant, at least for the SUM function ${ }^{1}$ in networks with general topologies: As the central contribution of this work, we prove that there exists (at least) an exponential gap between the non-fault-tolerant (NFT) and fault-tolerant (FT) communication complexity of SUM. Here FT communication complexity is the smallest communication complexity among all fault-tolerant protocols that can tolerate an arbitrary number of failures, while NFT communication complexity corresponds to all protocols. To our knowledge, ours is the first such result on FT communication complexity. This exponential gap attests that FT communication complexity needs to be studied separately from NFT communication complexity.

The Sum function. Consider a synchronous wireless network (e.g., a wireless sensor network or a wireless ad-hoc network) with $N$ nodes and some arbitrary topology. Each node has a binary value, and the Sum function asks for the sum of all the values. Note that SUM can be easily reduced to and from some other interesting aggregation functions such as SELECTION. Communication complexity has significant practical relevance here since i) wireless communication usually consumes far more energy than local computation, and needs to be minimized for nodes operating on battery power or nodes relying on energy harvesting, and ii) the capacity of wireless networks does not scale well [18].

Existing results on SUM. In failure-free settings, by leveraging in-network processing, a trivial tree-aggregation protocol can compute SUM with zero-error while requiring each node to send $O(\log N)$ bits. For $(\epsilon, \delta)$ approximate results, it is possible to further reduce to $O\left(\log \frac{1}{\epsilon}+\log \log N\right)$ bits per node for constant $\delta$. In comparison, to tolerate arbitrary failures, we are not aware of any zero-error protocol for computing Sum that is better than trivially having every node flood its id together with its value and thus requiring each node to send $O(N \log N)$ bits. For $(\epsilon, \delta)$-approximate results, researchers have proposed some protocols $[5,13,25,26,31]$ where each node needs to send roughly $O\left(\frac{1}{\epsilon^{2}}\right)$ bits for constant $\delta$ (after omitting logarithmic terms of $\frac{1}{\epsilon}$ and $N$ ). All these protocols conceptually map the value of each node to exponentially weighted positions in some bit vectors, and then estimate the sum from the bit vectors. Same as in one-pass distinct element counting algorithms in streaming databases [1,16], doing so makes the whole process duplicate-insensitive. In turn, this allows each node to push its value along multiple directions to guard against failures. Note however, that duplicate-insensitive techniques do not need to be one-pass, and furthermore tolerating failures does not have to use duplicate-insensitive techniques. For example, one could

\footnotetext{
${ }^{1}$ As an example where the impact of failures is not significant, consider the MAX function. Appendix A gives a simple folklore fault-tolerant MAX protocol based on binary search, where each node sends only a logarithmic number of bits.
} 

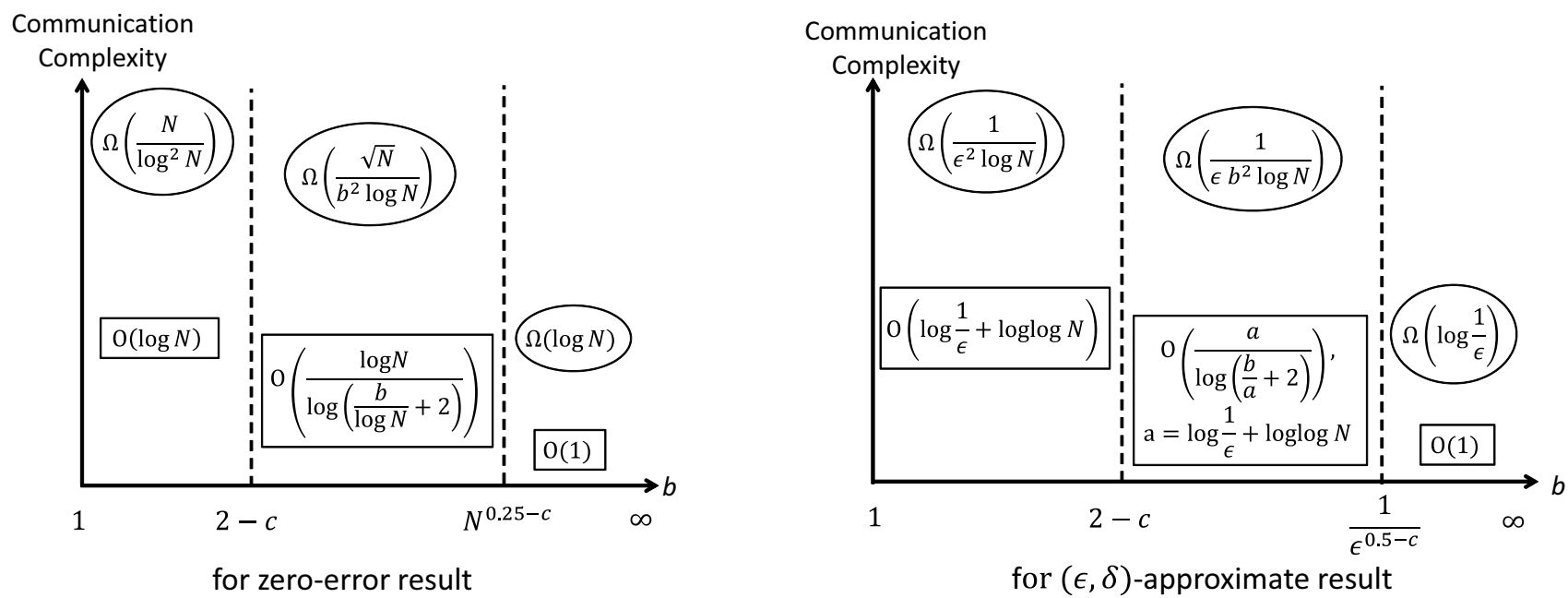

$b$ : time complexity of the protocol, in terms of the number of aggregation rounds $c$ : any positive constant below 0.25

$N$ : number of nodes in the network

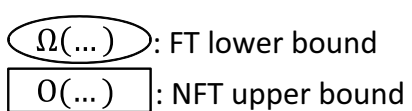

Figure 1: Summary of our exponential gaps. All NFT upper bounds are either well-known or are obtained via standard tricks - they are described in Section 3. All FT lower bounds are novel and are our main contributions. They are obtained in Section 4 (for $1 \leq b \leq 2-c$ ), Section 5 (for $2-c<b \leq N^{0.25-c}$ or $\left.\frac{1}{\epsilon^{0.5-c}}\right)$, and Section 7 (for $b>N^{0.25-c}$ or $\left.\frac{1}{\epsilon^{0.5-c}}\right)$.

repeatedly invoke the tree-aggregation protocol until one happens to have a failure-free run. There is also a large body of work $[3,7,11,12,20,22,23]$ on computing SUM via gossip-based averaging (also called average consensus protocols). They all rely on the mass conservation property [23], and thus are vulnerable to node failures. There have been a few efforts $[15,21]$ on making these protocols fault-tolerant. But they largely focus on correctness, without formal results on the protocol's communication complexity in the presence of failures. Despite all these efforts, no lower bounds on the FT communication complexity of SUM have ever been obtained, and thus it has been unknown whether the existing protocols can be improved.

Our results. Our main results in this paper are the first lower bounds on the FT communication complexity (or FT lower bounds in short) of SUM, for public-coin randomized protocols with zero-error and with $(\epsilon, \delta)$-error. These FT lower bounds are (at least) exponentially larger than the corresponding upper bounds on the NFT communication complexity (or NFT upper bounds in short) of SUM, thus establishing an exponential gap. Private-coin protocols and deterministic protocols are also fully but implicitly covered, and our exponential gap still applies.

Specifically, since there is a tradeoff between communication complexity and time complexity, Figure 1 summarizes our FT lower bounds when the time complexity of the SUM protocol is within $b$ aggregation rounds (defined in Section 2), for $b$ from 1 to $\infty$. For $b \leq N^{0.25-c}$ or $\frac{1}{\epsilon^{0.5-c}}$ where $c$ is any positive constant below 0.25 , the NFT upper bounds are always at most logarithmic with respect to $N$ or $\frac{1}{\epsilon}$, while the FT lower bounds are always polynomial. ${ }^{2}$ For $b>N^{0.25-c}$ or $\frac{1}{\epsilon^{0.5-c}}$, the NFT upper bounds drop to $O(1)$, while the FT lower bounds are still at least logarithmic. Our results also imply that under small $b$ values, the existing fault-tolerant Sum protocols (incurring $O(N \log N)$ or $O\left(\frac{1}{\epsilon^{2}}\right)$ bits $[5,13,25,26,31]$ per node) are actually optimal within polylog factors.

Our approach. Our FT lower bounds for $b \leq 2-c$ are obtained via a simple but interesting reduction

\footnotetext{
${ }^{2}$ Here for $(\epsilon, \delta)$-approximate results, we only considered terms containing $\epsilon$. Even if we take the extra terms with $N$ into account, our exponential gaps continue to exist as long as $\frac{1}{\epsilon^{c}}=\Omega(\log N)$.
} 
from a two-party communication complexity problem UNIONSIZE, where Alice and Bob intend to determine the size of the union of two sets. In the reduction, without knowing Bob's input, Alice can only simulate the SUM oracle protocol's execution in part of the network. Furthermore this part is continuously shrinking due to the spreading of such unknown information. Failures play a fundamental role in the reduction they hinder the spreading of unknown information. The FT lower bounds under $b \leq N^{0.25-c}$ or $\frac{1}{\epsilon^{0.5-c}}$ are much harder to obtain. There we introduce a new two-party problem called UNIONSIZECP, which is roughly UNIONSIZE extended with a novel cycle promise. Identifying this promise is a key contribution of this work, which enables the continuous injection of failures to further hinder the spreading of unknown information. We then prove a lower bound on UNIONSIZECP's communication complexity via information theoretic arguments [4]. This lower bound, coupled with our reduction, leads to FT lower bounds for SUM. We further prove a strong completeness result showing that UNIONSIZECP is complete among the set of all two-party problems that can be reduced to SUM in the FT setting via oblivious reductions (defined in Section 6). Namely, we prove that every problem in that set can be reduced to UNIONSIZECP. Our proof also implicitly derives the cycle promise, thus showing that it likely plays a fundamental role in reasoning about the FT communication complexity of many functions beyond SUM. Finally, our FT lower bounds under $b>N^{0.25-c}$ or $\frac{1}{\epsilon^{0.5-c}}$ are obtained by drawing a strong connection to an interesting probing game, and then by proving a lower bound on the probing game.

Other related work. Despite the developments (e.g., $[8,10,19,28,29])$ on different models for communication complexity, to the best of our knowledge, fault tolerance has never been considered. Among them, the closest setting to fault tolerance is perhaps unreliable channels $[8,28,29]$ that either flip the bits adversarially or flip each bit iid. The specific techniques and insights there have limited applicability to our faulttolerant setting. Under the iid unreliable channel model, there have also been some information-theoretic lower bounds $[2,17]$ on the rates of distributed computations. We suspect that such a lack of prior work on fault tolerance is due to two reasons. First, one needs to define correctness in a meaningful way when failures are possible, since some of the inputs can be missing. For this work, recent applications in wireless sensor networks have shown us how to do so [5]. Second, communication complexity problems tend to be challenging to study, and taking failures into account only makes things harder. For this work, we rely on several quite recent results $[9,19]$.

\section{Model and Definitions}

This section describes the system model and formal definitions used throughout this paper, except in Section 8. For clarity, we defer to Section 8 various relaxed/extended versions of the system model and definitions, under which our exponential gap results continue to hold. All "log" in this paper means $\log _{2}$.

System model. We consider a wireless network with $N$ nodes and an arbitrary undirected and connected graph $G$ as the network topology. Each node has a unique id, and one of the $N$ nodes is the root. We assume that the topology $G$ (including the ids of each node in $G$ ) is known to all nodes. The system is synchronous and a protocol proceeds in synchronous rounds. In each round, a node (which has not failed) first performs some local computation, and then does either a send or receive operation (but not both). We also say that the node is in a sending state or a receiving state in that round, respectively. Our results are insensitive to whether collisions are possible, but to make everything concrete, we still adopt and stick to the following commonlyused collision model. By doing a send, a node (locally) broadcasts one message to all its neighbors in $G$. By doing a receive, the node receives the message sent by one of its neighbors $j$ iff node $j$ is the only sending node among all node $i$ 's neighbors. If multiple neighbors of $i$ send in the same round, a collision occurs and node $i$ does not receive anything. All our results hold regardless of whether node $i$ can distinguish silence from collision.

Failure model. The root never fails. Any other node in $G$ may experience crash failures (but not byzantine 
failure), and the total number of failures can be up to $N-1$. See Section 8 for more discussion on the number of failures. To model worst-case behavior, we have an adversary determine which nodes fail at what time. The adversary can be adaptive to the behavior of the protocol (including the coin flips) so far, but it cannot predict future coin flip results.

The Sum problem. Here each node $i$ in $G$ has a binary value $w_{i}$, which is initially unknown to any other node. Let $s_{2}=\sum_{i=1}^{N} w_{i}$, and let $s_{1}$ be the sum of $w_{j}$ 's where by the end of the protocol's execution, node $j$ has not failed or been disconnected from the root due to other nodes' failures. Following the same definitions from [5], a zero-error result of SUM is any $s$ where $s_{1} \leq s \leq s_{2}$, and an $(\epsilon, \delta)$-approximate result of SUM is any $\hat{s}$ such that for some zero-error result $s, \operatorname{Pr}[|\hat{s}-s| \geq \epsilon s] \leq \delta$.

Time complexity of SUM protocols. We will consider only public-coin randomized protocols. By default, a "randomized protocol" in this paper is a public-coin randomized protocol. For a randomized Sum protocol and with respect to a topology $G$, we define the protocol's time complexity under $G$ to be the number of rounds needed for the protocol to terminate, under the worst-case values of the nodes in $G$, the worst-case failures (for fault-tolerant cases), and the worst-case random coin flips in the protocol. The topology $G$ has a large impact on time complexity, and we use the notion of aggregation rounds to isolate such impact. We will describe the time complexity in terms of aggregation rounds. This is analogous to describing it as a multiple of, for example, the diameter of $G$.

In failure-free settings, an aggregation round in $G$ consists of $\Lambda(G)$ rounds, where $\Lambda(G)$ is a function of the connected graph $G$. We will define $\Lambda(G)$ precisely later in Section 3, which describes a simple deterministic tree-aggregation protocol and then defines $\Lambda(G)$ as the number of rounds needed for that protocol to finish on $G$. When failures are possible, the network topology may change during execution. Let $\mathcal{G}$ be the set of all topologies that have ever appeared during the given execution. Note that a $G^{\prime} \in \mathcal{G}$ may or may not be connected. For any such $G^{\prime}$ that is not connected, we define $\Lambda\left(G^{\prime}\right)$ to be $\Lambda\left(G^{\prime \prime}\right)$ where $G^{\prime \prime}$ is the connected component of $G^{\prime}$ that contains the root. To allow a fair comparison between NFT and FT communication complexity, we define an aggregation round in an execution with failures to be $\max _{G^{\prime} \in \mathcal{G}} \Lambda\left(G^{\prime}\right)$ rounds. This implies that an aggregation round for an FT protocol is either the same or longer than that for an NFT protocol, which makes our gap results stronger.

NFT and FT communication complexity of SUM protocols. Classic multi-party communication complexity problems [24] usually consider the total number of bits sent by all players, since they usually use the whiteboard model where the whiteboard is the bottleneck. In our distributed computing setting with a topology $G$, as in other problems in such a setting, it is more natural to consider the number of bits sent by the bottleneck player. Given a randomized Sum protocol, a topology $G$, a value assignment to the nodes in $G$, and a failure adversary (if failures are considered), define $a_{i}$ to be the expected (with the expectation taken over coin flips in the protocol) number of bits that node $i$ sends. The protocol's average-case communication complexity under $G$ is defined as the largest $a_{i}$, across all value assignments of the nodes in $G$, all failure adversaries (if failures are considered), and all $i$ 's $(1 \leq i \leq N)$. The protocol's worst-case communication complexity under $G$ is similarly defined by considering worst-case coin flips instead of taking the expectation over the coin flips.

We define $\mathcal{R}_{0}^{\text {syn }}$ (SUM, $\left.G, b\right)\left(\mathcal{R}_{\epsilon, \delta}^{\text {syn }}\right.$ (SUM, $\left.G, b\right)$, respectively) to be the smallest average-case (worstcase, respectively) communication complexity under $G$ across all randomized Sum protocols that can generate, in a failure-free setting, a zero-error result $((\epsilon, \delta)$-approximate result, respectively) on $G$ within a time complexity of at most $b$ aggregation rounds. Here note that i) the length of an aggregation round depends on $G$, and ii) using the worst-case communication complexity for defining $\mathcal{R}_{\epsilon, \delta}^{\text {syn }}$ is standard practice [4, 24]. With respect to any topology $G$, we similarly define $\mathcal{R}_{0}^{\text {syn,ft }}$ (SUM, $\left.G, b\right)$ and $\mathcal{R}_{\epsilon, \delta}^{\text {syn,ft }}$ (SUM, $\left.G, b\right)$ across all fault-tolerant randomized SUM protocols.

For any given integer $N$, we define SUM's NFT communication complexity $\mathcal{R}_{0}^{\mathrm{Syn}}\left(\mathrm{SUM}_{N}, b\right)$ and $\mathcal{R}_{\epsilon, \delta}^{\mathrm{Syn}}\left(\mathrm{SuM}_{N}, b\right)$ to be the maximum $\mathcal{R}_{0}^{\mathrm{Syn}}(\mathrm{SUM}, G, b)$ and $\mathcal{R}_{\epsilon, \delta}^{\mathrm{Syn}}$ (SUM, $\left.G, b\right)$, respectively, across all topol- 
ogy $G$ 's where $G$ is connected and has exactly $N$ nodes. Similarly define SuM's FT communication complexity $\mathcal{R}_{0}^{\mathrm{syn}, \mathrm{ft}}\left(\mathrm{SuM}_{N}, b\right)$ and $\mathcal{R}_{\epsilon, \delta}^{\mathrm{syn}, \mathrm{ft}}\left(\mathrm{Sum}_{N}, b\right)$.

Communication complexity of two-party problems. Our proofs will also need to reason about the NFT communication complexity of some two-party problems. In such a problem $\Pi$, Alice and Bob each have an input $X$ and $Y$ respectively, and the goal is to compute the function $\Pi(X, Y)$. For all two-party problems in this paper, we only require Alice to learn the final result. We will often use $n$ to denote the size of $\Pi$, as compared to $N$ which describes the number of nodes in $G$. The communication complexity of a randomized protocol for computing $\Pi$ is defined to be either the average-case or worst-case (over random coin flips) number of bits sent by Alice and Bob combined. In the classic setting without synchronous rounds [24], similar as earlier, we define $\mathcal{R}_{0}(\Pi)\left(\mathcal{R}_{\epsilon, \delta}(\Pi)\right.$, respectively) to be the smallest average-case (worst-case, respectively) communication complexity across all randomized protocols that can generate a zero-error result $((\epsilon, \delta)$-approximate result, respectively) for $\Pi$. We will also need to consider a second setting with synchronous rounds ${ }^{3}$, adapted from [19]. Here Alice and Bob proceed in synchronous rounds, where in each round Alice and Bob may simultaneously send a message to the other party. Alice, or Bob, or both may also choose not to send a message in a round. The time complexity of a randomized protocol for computing $\Pi$ is defined to be the number of rounds needed for the protocol to terminate, over the worst-case input and the worst-case coin flips. We define $\mathcal{R}_{0}^{\mathrm{syn}}(\Pi, t)\left(\mathcal{R}_{\epsilon, \delta}^{\mathrm{syn}}(\Pi, t)\right.$, respectively) to be the smallest average-case (worst-case, respectively) communication complexity across all randomized protocols for $\Pi$ that can generate a zero-error result $((\epsilon, \delta)$-approximate result, respectively) within a time complexity of at most $t$ rounds.

\section{Upper Bounds on NFT Communication Complexity of SUM}

This section describes the NFT upper bounds on SUM, which are from well-known tree-aggregation protocols coupled with some standard tricks. These are not our main contribution - instead, they serve to show the exponential gap from our FT lower bounds.

Tree-aggregation protocol and defining $\Lambda(G)$. Since the topology $G$ is known, every node can locally and deterministically construct a breadth-first spanning tree (with the root of $G$ being the tree root) as the aggregation tree. With this tree in place, a node becomes ready when it receives one aggregation message from each of its children. Each aggregation message encodes the partial sum of all the values in the corresponding subtree. Leaf nodes are ready from the beginning. A ready node will combine all these aggregation messages, together with its own value, and then send a single aggregation message to its parent. With the known topology, the protocol easily avoids collision via the following simple deterministic scheduling: Out of all ready nodes, the protocol greedily and deterministically chooses a maximal set of nodes to send messages without incurring collision. A message does not need to include the sender's id - since everything is deterministic, the receiver can locally determine the sender. The function $\Lambda(G)$ is formally defined to be the number of rounds needed for the above deterministic protocol to finish on $G$. Thus by definition, the above protocol has a time complexity of one aggregation round.

NFT upper bounds. If each aggregation message uses $O(\log N)$ bits to encode the exact partial sum, then the above protocol is a deterministic protocol for SuM with $O(\log N)$ communication complexity and one aggregation round time complexity. For $(\epsilon, \delta)$-approximate results, it is possible to reduce the size of the aggregation message to $O\left(\log \frac{1}{\epsilon}+\log \log N\right)$ bits, using a simple private-coin protocol with similar tricks as in AMS synopsis [1] (see Appendix B). One can further reduce the communication complexity if the time complexity is $b$ aggregation rounds with $b>1$, since we can now spend $b$ rounds in sending all the bits previously sent in one round. It is known [19] that an $a$-bit message sent in one round can be encoded using

\footnotetext{
${ }^{3}$ These synchronous rounds are different from interaction rounds, which correspond to message exchanges. A protocol using $x$ synchronous rounds incurs $x$ or fewer interaction rounds since a synchronous round may or may not have any message.
} 
$a / \log \frac{b}{a}$ bits sent over $b$ rounds, for $b \geq 2 a$. To do so, one bit is sent every $\frac{b}{a} \cdot \log \frac{b}{a}$ rounds. Leveraging the round number during which the bit is sent, each such bit can encode $\log \left(\frac{b}{a} \cdot \log \frac{b}{a}\right) \geq \log \frac{b}{a}$ bits of information. Combining all the above leads to:

Theorem 1 For any $b \geq 1$, we have:

$$
\begin{aligned}
& \mathcal{R}_{0}^{\mathrm{Syn}}\left(\operatorname{SuM}_{N}, b\right)=O\left(a / \log \left(\frac{b}{a}+2\right)\right), \quad \text { where } a=\log N \\
& \mathcal{R}_{\epsilon, \frac{1}{3}}^{\mathrm{Syn}}\left(\operatorname{SuM}_{N}, b\right)=O\left(a / \log \left(\frac{b}{a}+2\right)\right), \quad \text { where } a=\log \frac{1}{\epsilon}+\log \log N
\end{aligned}
$$

\section{Lower Bounds on FT Communication Complexity of SUM for $b \leq 2-c$}

The UnionSize problem. As discussed in Section 1, one possible approach to achieve fault tolerance when computing SUM is for the nodes to simultaneously propagate their values along multiple directions. But doing so will lead to duplicates which must be addressed. Thus it is natural to consider a potential reduction from the two-party communication complexity problem UNIONSIZE, which was used for obtaining the optimal $\Omega\left(\frac{1}{\epsilon^{2}}\right)$ lower bound on the space complexity of one-pass distinct element counting [30]. In the two-party problem UnIONSIZE ${ }_{n}$, Alice and Bob have length- $n$ binary strings $X$ and $Y$, respectively. Let $X_{i}\left(Y_{i}\right)$ denote the $i$ th bit of $X(Y)$. Alice aims to determine $\mid\left\{i \mid X_{i} \neq 0\right.$ or $\left.Y_{i} \neq 0\right\} \mid$. If $X$ and $Y$ are the characteristic vectors of two sets, then this is the size of the union of the two sets. Trivially combining a few recent results $[9,19,30]$ tells us that $\mathcal{R}_{0}^{\text {Syn }}\left(\operatorname{UNIONSIZE}_{n}, O(\operatorname{poly}(n))\right)=\Omega\left(\frac{n}{\log n}\right)$ and $\mathcal{R}_{\epsilon, \frac{1}{3}}^{\text {syn }}\left(\operatorname{UnIONSIZE}_{n}, O(\operatorname{poly}(n))\right)=\Omega\left(\frac{1}{\epsilon^{2} \log n}\right)$ for $\epsilon=\Omega\left(\frac{1}{\sqrt{n}}\right)$ (see Corollary 10 in Appendix A).

Overview of our reduction and its novelty. While the well-known reduction [30] from UNIONSIZE to the (centralized) one-pass distinct element counting problem is almost trivial, we seek a reduction from UNIONSIZE to SUM, which is less obvious. In particular, it is not immediately clear what a role failures can play. Our simple yet interesting reduction here will answer this question, which prepares for our trickier reduction in Section 5. Our reduction is based on a certain topology $G$. Given inputs $X$ and $Y$ to UnIOnSizE, each node in $G$ has some value so that their sum is exactly $\operatorname{UniOnSiZE}(X, Y)$. The values of some of the nodes are uniquely determined by $X$, and thus are known by Alice from her local knowledge of $X$. If the value of a node $\tau$ cannot be uniquely determined by $X$, then $\tau$ is spoiled (rigorously defined in Appendix C.2) for Alice, in the sense that Alice cannot simulate $\tau$. As the simulation proceeds, a spoiled node $\tau$ may causally affect its neighbor node $\tau^{\prime}$, rendering Alice unable to simulate $\tau^{\prime}$ and thus making $\tau^{\prime}$ spoiled as well. Since the Sum protocol may have internal state, if Alice cannot simulate a node for some round, then Alice cannot simulate the node for later rounds either. In this sense, a spoiled node can never get "unspoiled" later. For each round, Alice will simply simulate the (shrinking) group of all those nodes that have not been spoiled for Alice. Bob similarly simulates all unspoiled nodes for Bob. Alice's group and Bob's may intersect.

We want the root of $G$ to remain unspoiled for Alice when the Sum protocol ends, so that it provides the Sum result to Alice for her to determine $\operatorname{UnionSize}(X, Y)$. To achieve this, in the reduction, Alice and Bob will need to strategically simulate the failures of certain nodes, to block the spreading of spoiled nodes. This showcases the fundamental role of failures in our reduction. At the same time, we need to avoid failing/disconnecting nodes with a value of 1 - failing/disconnecting them would enable the SUM protocol to ignore their values and potentially return a result that cannot be used to determine $\operatorname{UnIONSiZE}(X, Y)$. (Recall from Section 2 that a zero-error result for Sum can be any value between $s_{1}$ and $s_{2}$.) In fact, if we were not concerned with this, then simply failing all nodes except the root would keep the root unspoiled forever. Finally, it is also necessary to enlist help from Bob, who can simulate certain nodes that are spoiled for Alice. By forwarding to Alice messages sent by those nodes, Bob can further hinder the shrinking of Alice's group. The communication (between Alice and Bob) spent in doing so will be the communication 


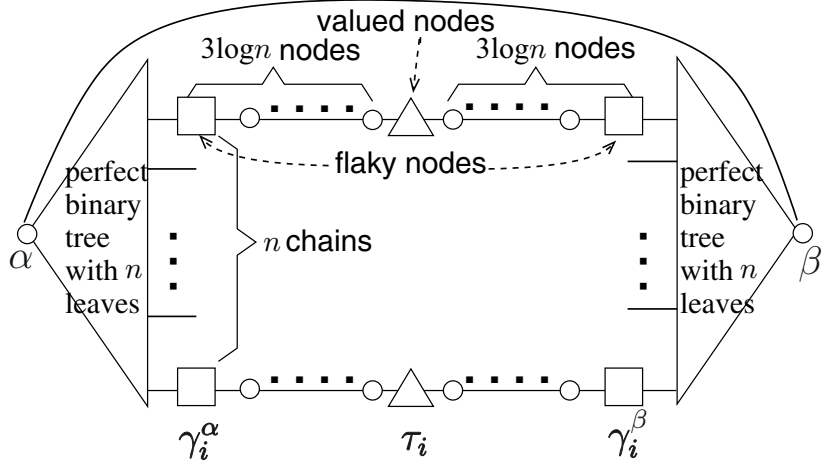

Figure 2: FT lower bound topology for $b \leq \frac{10}{9}$.

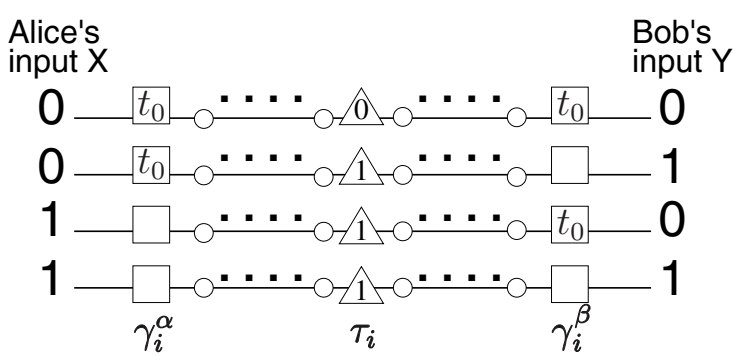

Figure 3: Values of valued nodes and failure times of flaky nodes, for $X=0011$ and $Y=$ 0101.

complexity incurred for solving UNIONSIZE. Simulating a shrinking group of nodes and properly using failures to hinder such shrinking is the main novelty in our simple reduction.

Reducing from UnIONSIZE to SUM. For better understanding, the topology (Figure 2) we describe here works for $b \leq \frac{10}{9}$. See Appendix $C$ for the topology for $b \leq 2-c$, with the $c$ there being any positive constant. Given UNIONSIZE $n$ with $n$ being a power of 2, the topology here has $n$ parallel chains of nodes. Each chain has $6 \log n+1$ nodes. We use $\gamma_{i}^{\alpha}, \tau_{i}$, and $\gamma_{i}^{\beta}$ to denote the first, middle, and last node on the $i$ th chain, respectively. Next we construct a perfect binary tree with all the $\gamma_{i}^{\alpha}$ 's being the leaves, and let node $\alpha$ denote the tree root. Similarly construct a second perfect binary tree whose leaves are all the $\gamma_{i}^{\beta}$,s, and let $\beta$ be the tree root. Finally, we connect $\alpha$ and $\beta$ with a single edge, and let $\alpha$ be the root of the topology. This topology has total $N=\Theta(n \log n)$ nodes.

The inputs $X$ and $Y$ to $\mathrm{UNIONSIZE}_{n}$ will determine the values of the $\tau_{i}$ 's, which are called valued nodes. Specifically, $\tau_{i}$ has a binary value of 1 iff $X_{i} \neq 0$ or $Y_{i} \neq 0$ (Figure 3). All other nodes (i.e. non-valued nodes) have values of $0 . X$ and $Y$ also determine the failure times of the $\gamma_{i}^{\alpha}$,s and $\gamma_{i}^{\beta}$,s, which are called flaky nodes. If $X_{i}=0$, then $\gamma_{i}^{\alpha}$ fails at the beginning of round $t_{0}=3 \log n+1$. Otherwise it never fails. Intuitively, $t_{0}$ is the very first round where $\tau_{i}$ may causally affect $\gamma_{i}^{\alpha}$. Similarly, $\gamma_{i}^{\beta}$ fails at the beginning of round $t_{0}$ iff $Y_{i}=0$ (Figure 3). Non-flaky nodes never fail. It is worth noting that this failure adversary i) is oblivious to the SUM protocol, and ii) fails only a vanishingly small fraction (i.e., $o(N)$ ) of all the nodes in $G$.

As a key property in the above construction (and later constructions), a $\tau_{i}$ whose value is 1 is never disconnected from the root. This is because if $\tau_{i}$ 's value is 1 , then it must be unspoiled (by our construction) for either Alice or Bob, and thus can remain connected to $\alpha$ or $\beta$ (and thus to the root). This in turn ensures that a zero-error result of $\operatorname{SUM}$ is always exactly $\operatorname{UniONSIZE}(X, Y)$.

Alice will simulate the shrinking group of all the unspoiled nodes for Alice, which always contains node $\alpha$. Bob similarly simulates the unspoiled nodes for Bob, including node $\beta$. (These two groups are made precise in Appendix C.) Whenever $\alpha$ in the SuM protocol sends a message (whose intended recipient may or may not be $\beta$ ) Alice always forwards that message to Bob. Bob does the same whenever $\beta$ sends a message. Alice and Bob do not exchange any additional messages. Thus the number of bits sent by Alice and Bob for solving UNIONSIZE is exactly the same as the number of the bits sent by $\alpha$ and $\beta$ in the Sum protocol.

To obtain some intuition, let us consider some $i$ where $X_{i}=0$ and $Y_{i}=1$. This makes $\tau_{i}$ spoiled for Alice, since Alice cannot determine $\tau_{i}$ 's value based on $X_{i}$. To prevent $\tau_{i}$ from causally affecting $\alpha$ and thus spoiling $\alpha$, Alice simulates the failure of $\gamma_{i}^{\alpha}$ before this can happen. Interestingly, since based on $Y_{i}$ Bob cannot determine whether $\gamma_{i}^{\alpha}$ fails, $\gamma_{i}^{\alpha}$ becomes spoiled for Bob when it fails. Once the failure of $\gamma_{i}^{\alpha}$ can causally affect $\beta$ (at round $10 \log n+1$ ), Bob can no longer simulate $\beta$. The simulation must end before this happens, which is guaranteed under $b \leq \frac{10}{9}$ since an aggregation round here has no more than $8 \log n+1$ rounds. 
We obtain the following theorem by formalizing the above arguments, using an improved topology as in Appendix $\mathrm{C}$, and then trivially extending to those $N$ values that currently do not map to any integer $n$ for UNIONSIZE $_{n}$. The proof is in Appendix C.

Theorem 2 For any $b \in[1,2-c]$ where $c$ is any positive constant, we have:

$$
\begin{aligned}
& \mathcal{R}_{0}^{\mathrm{syn}, \mathrm{ft}}\left(\mathrm{SuM}_{N}, b\right)=\Omega\left(\frac{N}{\log ^{2} N}\right) \\
& \mathcal{R}_{\epsilon, \frac{1}{3}}^{\mathrm{syn}, \mathrm{ft}}\left(\operatorname{SuM}_{N}, b\right)=\Omega\left(\frac{1}{\epsilon^{2} \log N}\right), \text { for } \epsilon=\Omega\left(\frac{\sqrt{\log N}}{\sqrt{N}}\right)
\end{aligned}
$$

\section{Lower Bounds on FT Communication Complexity of SUM for $b \leq N^{0.25-c}$ or $1 / \epsilon^{0.5-c}$}

Why the previous construction cannot be extended. The FT lower bounds in the previous section no longer hold for larger $b$ since the failure of $\gamma_{i}^{\alpha}$ (as simulated by Alice) makes $\gamma_{i}^{\alpha}$ spoiled for Bob, which will in turn spoil $\beta$ under larger $b$. A natural attempt to fix this is to inject new failures to prevent such propagation of spoiled nodes, as in Figure 4. Here when $Y_{i}=1$, Bob simulates a new failure to the left of $\tau_{i}$, to prevent the propagation of spoiled nodes due to $\gamma_{i}^{\alpha}$. This new failure cannot be to the right of $\tau_{i}$ because otherwise when $X_{i}=0$ (implying the failure of $\gamma_{i}^{\alpha}$ ) and $Y_{i}=1, \tau_{i}$ has a value of 1 and is disconnected from the root. As explained in Section 4, this prevents us from using the SUM result to determine UnionSizE. Similarly, Alice needs to simulate a new failure on the right side of $\tau_{i}$, when $X_{i}=1$. This eventually implies that when $X_{i}=Y_{i}=1$, both of these two new failures will be introduced, again disconnecting $\tau_{i}$. One could avoid this problem by adding a promise and disallowing $X_{i}$ and $Y_{i}$ to simultaneously be 1 . Unfortunately, such a naive promise decreases the communication complexity of $\mathrm{UNIONSIZE}_{n}$ to $O(\log n)$, making the final results trivial.

The UnionSizeCP problem. To overcome the above problem, we will introduce and reduce from a new two-party communication complexity problem called UNIONSIZECP. UNIONSIZECP is intuitively UNIONSIZE extended with a novel promise which we call the cycle promise. This promise is not constructed ad hoc - rather, we will later see that it can be derived. In $\mathrm{UnIONSIZECP}_{n, q}$ where $q \geq 2$, Alice and Bob respectively have length- $n$ strings $X$ and $Y$. The characters in the strings are integers in $[0, q-1]$. Let $X_{i}$ and $Y_{i}$ denote the $i$ th character of $X$ and $Y$, respectively. $X$ and $Y$ satisfy the following cycle promise where for all $i$ : If $X_{i}=0$, then $Y_{i}$ must be 0 or 1 ; if $X_{i}=q-1$, then $Y_{i}$ must be $q-2$ or $q-1$; if $0<X_{i}<q-1$, then $Y_{i}$ must be $X_{i}-1$ or $X_{i}+1$. This promise is illustrated in Figure 5 as a bipartite promise graph, where values for $X_{i}$ and $Y_{i}$ are vertices and two values are connected by an edge if they satisfy the promise. Note

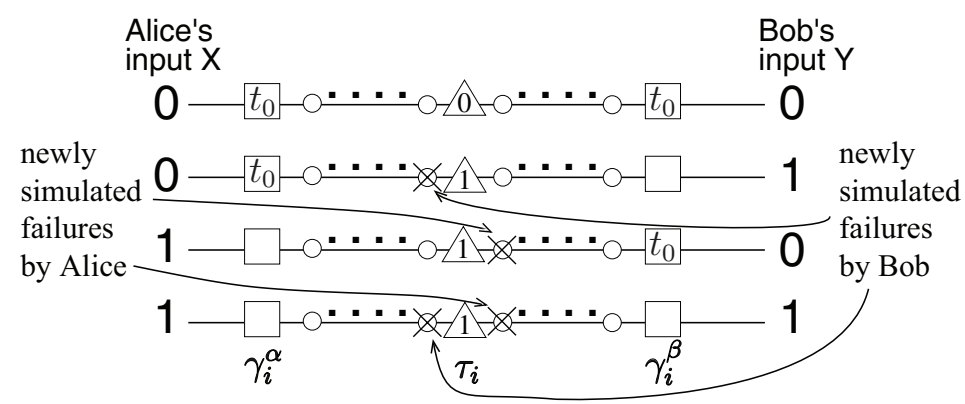

Figure 4: Why the construction from Section 4 cannot be extended to larger $b$.

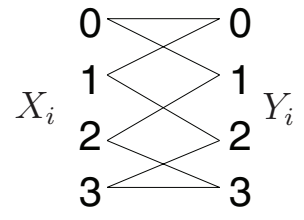

Figure 5: The cycle promise for $q=4$. 


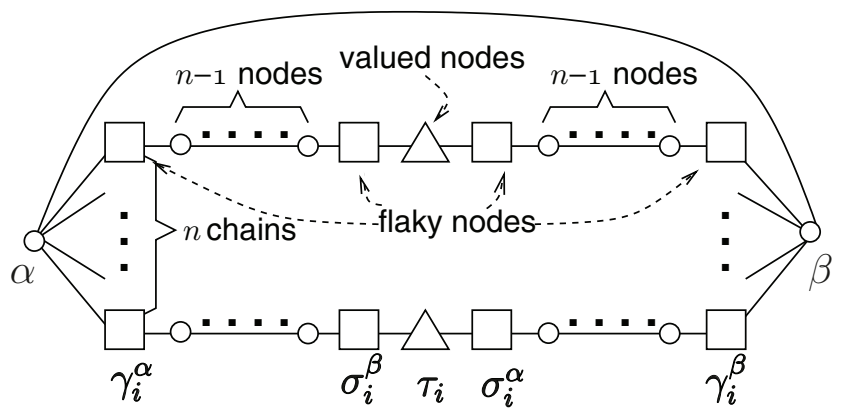

Figure 6: FT lower bound topology for $b \leq N^{0.25-c}$ or $b \leq \frac{1}{\epsilon^{0.5-c}}$.
Alice's

Bob's

input $X$ input $Y$
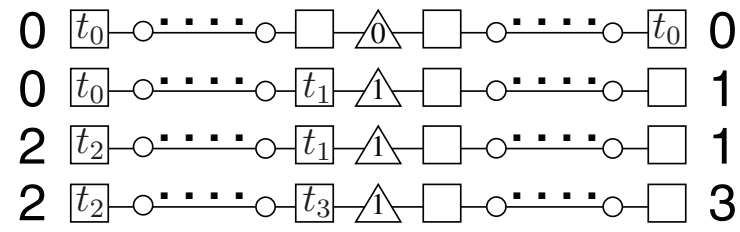

1

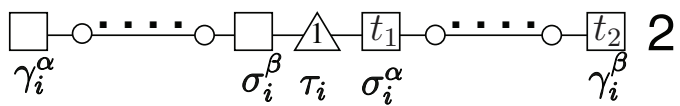

Figure 7: Values of valued nodes and failure times of flaky nodes, for $q=4, X=00221$, and $Y=01132$.

that this promise graph is actually a cycle. Same as in UnIONSize, the goal in UnIONSizeCP is for Alice to determine $\mid\left\{i \mid X_{i} \neq 0\right.$ or $\left.Y_{i} \neq 0\right\} \mid$. When $q=2$, UnIONSIZECP degrades to UnIONSIZE. Later we will show that different from the earlier naive promise, the cycle promise does not make the communication complexity of UNIONSIZECP trivial. In our reduction to SUM, the cycle promise will enable us to continuously introduce new failures to block the spreading of spoiled nodes caused by old failures, without disconnecting any node in $G$ with a value of 1 . Those newly failed nodes then become spoiled themselves, requiring further failures to be injected, until the end of the simulation.

Reducing from UnionSizeCP to Sum. Figure 6 illustrates the topology used in our reduction from UNIONSIZECP $_{n, q}$, which has $n$ parallel chains of nodes, with each chain having $2 n+3$ nodes. We connect the first node of each chain directly to a node $\alpha$, and the last node of each chain directly to a node $\beta{ }^{4}$ Finally, we connect $\alpha$ and $\beta$ with a single edge, and let $\alpha$ be the root of the topology. This topology has total $N=\Theta\left(n^{2}\right)$ nodes. As before, Alice (Bob) will simulate a continuously shrinking group of nodes including $\alpha(\beta)$. As illustrated in Figure 7, the middle node $\tau_{i}$ of the $i$ th chain is a valued node whose value is 1 iff $X_{i} \neq 0$ or $Y_{i} \neq 0$. There are 4 flaky nodes on the chain from left to right: the first node of the chain, the two neighbors of $\tau_{i}$, and the last node of the chain. We use $\gamma_{i}^{\alpha}, \sigma_{i}^{\beta}, \sigma_{i}^{\alpha}$, and $\gamma_{i}^{\beta}$ to denote these 4 nodes, respectively. Let $t_{j}=(j+1) n+1$ for all $0 \leq j \leq q-1$. The flaky node $\gamma_{i}^{\alpha}$ fails at the beginning of round $t_{X_{i}}$ iff $X_{i}$ is even, while $\sigma_{i}^{\alpha}$ fails at the beginning of round $t_{X_{i}}$ iff $X_{i}$ is odd (Figure 7). Similarly, $\gamma_{i}^{\beta}\left(\sigma_{i}^{\beta}\right)$ fails at the beginning of round $t_{Y_{i}}$ iff $Y_{i}$ is even (odd). Again, the failure adversary here is oblivious to the Sum protocol, and fails only a vanishingly small fraction (i.e., $o(N)$ ) of all the nodes in $G$.

To gain some intuition, consider the example in Figure 8. We say that a node is an epicenter for Alice's input $X$ if it is a valued node (or a flaky node) whose value (or failure time) is not uniquely determined by $X$. Similarly define epicenters for Bob's input $Y$. Essentially, an epicenter is the source of the spreading of spoiled nodes. When $X_{i}=0, \tau_{i}$ is an epicenter for Alice and thus Alice simulates the failure of $\gamma_{i}^{\alpha}$ at $t_{0}$ to block the influence of such $\tau_{i}$ (i.e., the top/middle scenario in Figure 8). Next since the failure of $\gamma_{i}^{\alpha}$ depends on $X_{i}$ and is not uniquely determined by $Y$, the node $\gamma_{i}^{\alpha}$ itself now becomes an epicenter for Bob. With the cycle promise and since $X_{i}=0, Y_{i}$ must be 0 or 1 . If $Y_{i}=0$, then Bob does not need to be concerned, since Bob has already simulated the failure of $\gamma_{i}^{\beta}$ at $t_{0}$ and thus blocked the potential influence of $\gamma_{i}^{\alpha}$ (i.e., the top scenario). If $Y_{i}=1$ however, Bob needs to simulate the failure of $\sigma_{i}^{\beta}$ at $t_{1}$ (i.e., the middle scenario) to block the influence of $\gamma_{i}^{\alpha}$. Now $\sigma_{i}^{\beta}$ again, becomes an epicenter for Alice (i.e., the middle/bottom scenario). Given the cycle promise and since $Y_{i}=1$, we must have $X_{i}=0$ or $X_{i}=2$. If $X_{i}=0$, then Alice has already simulated the failure of $\gamma_{i}^{\alpha}$ at $t_{0}$ and has already blocked the potential influence of $\sigma_{i}^{\beta}$ (i.e., the middle

\footnotetext{
${ }^{4}$ Using binary trees will not work here. Consequently, here an aggregation round will contain more rounds than in Section 4, and in turn each chain needs to have more nodes.
} 


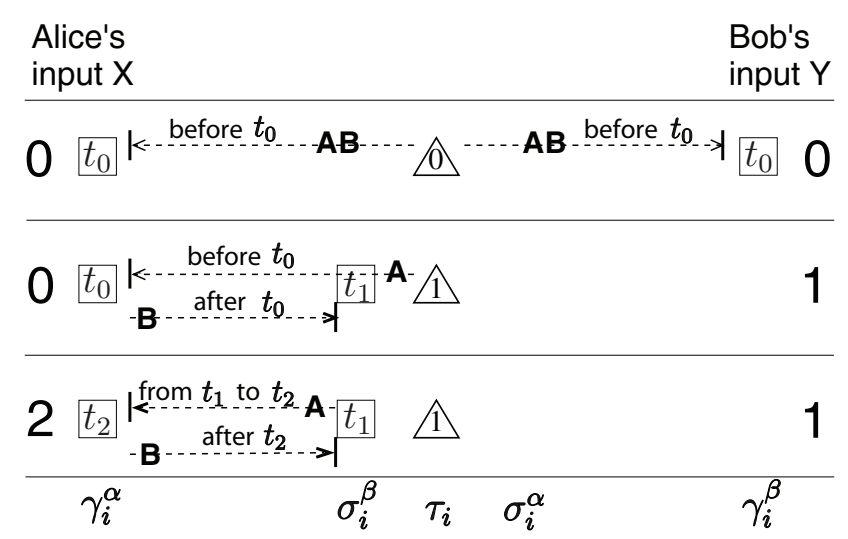

Figure 8: Failures prevent the spreading of spoiled nodes. Dashed arrows labeled $\mathbf{A}(\mathbf{B})$ indicate the spreading of spoiled nodes for Alice (Bob).

scenario). If $X_{i}=2$ however, Alice needs to simulate a new failure of $\gamma_{i}^{\alpha}$ at $t_{2}$ (i.e., the bottom scenario). Extending such reasoning can show that by continuously injecting new failures, we can always manage to block the spreading of spoiled nodes.

Finally, note that the simulation still cannot continue forever. Under the cycle promise, it is possible for $X_{i}=Y_{i}=q-1$. Thus we need the SUM protocol to stop by round $t_{q-1}-1$, since otherwise at the beginning of round $t_{q-1}$, Alice and Bob would simulate failures such that $\tau_{i}$ (with a value of 1) would be disconnected. This means that $q$ needs to be chosen based on the Sum protocol's time complexity $b$ : A larger $q$ is needed when $b$ is larger. Since the communication complexity of UnIONSIZECP depends on $q$ (as shown next), as expected, our lower bounds here will be a function of $b$. We obtain the following theorem via formalizing the above reduction, using our lower bound later (Theorem 4) from UNIONSIZECP, and then trivially extending to all $N$ values. See Appendix D for the proof.

Theorem 3 For any $b \geq 1$, we have:

$$
\begin{aligned}
& \mathcal{R}_{0}^{\mathrm{syn}, \mathrm{ft}}\left(\mathrm{Sum}_{N}, b\right)=\Omega\left(\frac{\sqrt{N}}{b^{2} \log N}\right) \\
& \mathcal{R}_{\epsilon, \frac{1}{5}}^{\mathrm{syn}, \mathrm{ft}}\left(\mathrm{SuM}_{N}, b\right)=\Omega\left(\frac{1}{\epsilon b^{2} \log N}\right), \text { for } \epsilon=\Omega\left(\frac{1}{\sqrt[4]{N}}\right)
\end{aligned}
$$

Communication complexity of UNIONSIZECP. Since UNIONSIZECP has never been studied, there are no existing results on its communication complexity. Proving these results is thus also a contribution of our work, which may be of independent interest. On the surface, it may appear that the complexity of UNIONSIZECP should not be very different from that of UnIONSIZE. This first thought turns out to be incorrect. For $q \leq n$, Appendix E presents an $O\left(\frac{n}{q}\right)$ upper bound protocol for $\mathcal{R}_{0}^{\mathrm{Syn}}\left(\operatorname{UNIONSIZECP}_{n, q}, \operatorname{poly}(n)\right)$, implying that its communication complexity drops at least linearly with $\frac{1}{q}$. In this protocol, Alice finds the integer $j$ with the smallest occurrence count in $X$, and sends Bob $j$ and the set $\left\{i \mid X_{i}=j\right\}$. This takes $O\left(\frac{n}{q} \log n\right)$ bits in one round, or $O\left(\frac{n}{q}\right)$ bits in poly $(n)$ rounds [19]. Now we only need to worry about indices not in the set. For those indices, the promise graph (Figure 5) degrades to a chain, since two edges are removed from the cycle. This makes the UNIONSIZECP problem easy to solve after we apply a mapping trick (see Appendix E). To lower bound UNIONSIZECP's communication complexity, we find that the cycle promise makes it challenging to apply classic arguments based on rectangles [24]. ${ }^{5}$ But we also find that UNIONSIZECP is rather amenable to information theoretical arguments [4], which lead to the following theorem whose proof is in Appendix E:

\footnotetext{
${ }^{5}$ Leveraging some strong results on the sperner capacity of the cyclic $q$-gon [6], we managed to obtain some results on $\mathcal{R}_{0}\left(\right.$ UnionSizeCP), but not on $\mathcal{R}_{\epsilon, \delta}($ UnionSIZECP $)$.
} 
Theorem 4 We have:

$$
\begin{aligned}
& \mathcal{R}_{0}^{\text {Syn }}\left(\operatorname{UniONSIZECP}_{n, q}, O(\operatorname{poly}(n))\right)=\Omega\left(\frac{n}{q^{2} \log n}\right) \\
& \mathcal{R}_{\epsilon, \frac{1}{5}}^{\text {syn }}\left(\operatorname{UniONSIZECP}_{n, q}, O(\operatorname{poly}(n))\right)=\Omega\left(\frac{1}{\epsilon q^{2} \log n}\right), \text { for } \epsilon=\Omega\left(\frac{1}{\sqrt{n}}\right)
\end{aligned}
$$

\section{The Fundamental Roles of Cycle Promise and UnionSizeCP}

Our reduction from UNIONSIZECP so far has led to the exponential gap result for SUM, when $b \leq N^{0.25-c}$ or $\frac{1}{\epsilon^{0.5-c}}$ for any positive constant $c<0.25$. This restriction on $b$ comes from the $\frac{1}{q^{2}}$ term in the lower bound of the communication complexity of UNIONSIZECP. Our upper bound on UNIONSIZECP indicates that such a polynomial dependency on $\frac{1}{q}$ is unavoidable because of the cycle promise. It is thus natural to ask: Can we reduce from problems without promises? Or can we reduce from problems with a different promise, to weaken the polynomial dependency on $\frac{1}{q}$ to $\log \frac{1}{q}$ ? For any possible oblivious reduction (defined next) from any two-party communication complexity problem $\Pi$ to SUM, this section answers these questions in the negative. Specifically, we prove the completeness of UnIONSIZECP in the sense that such a $\Pi$ can always be reduced to UNIONSIZECP and must have a communication complexity no larger than that of UNIONSIZECP $_{N,\lfloor\sqrt{b / 3}\rfloor}$. Thus any FT lower bound on SUM, obtained in such a way via $\Pi$, must contain some polynomial term of $\frac{1}{b}$. Overcoming this polynomial term in the lower bound might still be possible, but one would have to resort to methods other than oblivious reductions from two-party problems. Our proof also (implicitly) shows that the cycle promise can be derived and that the promise likely plays a fundamental role in reasoning about many functions beyond SUM.

Reductions and oblivious reductions. Consider any two-party communication complexity problem $\Pi$, where Alice aims to learn $\Pi(X, Y)$. In a (general) reduction from $\Pi$ to SuM, Alice and Bob are given some black-box oracle fault-tolerant protocol for SuM, and they are supposed to use this oracle to solve $\Pi$ with any given input pair $(X, Y)$. Since the (global) oracle protocol is distributed, it will be convenient to imagine that each node in the topology has its own oracle protocol, and invoking these protocols in a "consistent" fashion will enable the root to produce a meaningful result.

In an oblivious reduction to SUM, there is some fixed topology $G$ and for each $(X, Y)$ pair, there exists some reference setting specifying the value and failure time of each node in $G$. The reference settings are oblivious to the oracle. As explained in Section 4, a reference setting here should not fail or disconnect nodes with a value of 1 . The zero-error Sum result in the reference setting should be the same as $\Pi(X, Y)$, so we can directly use it for solving $\Pi$. The reduction protocol is required to be oblivious as well. Specifically, Alice and Bob first pick a (public) random string. Next before invoking the oracle and purely based on $X$ $(Y)$, Alice (Bob) decides for each node in $G$, exactly up to which round she (he) will invoke the oracle. Note that to invoke the oracle for a certain round, Alice/Bob needs to invoke the oracle for all previous rounds as well. Alice (Bob) also decides the (initial) value of each node for which she (he) will invoke the oracle for at least one round. Requiring Alice and Bob to make these decisions beforehand is the most important aspect of oblivious reductions. We define the reference execution for $(X, Y)$ to be the (global) oracle's execution under the reference setting for $(X, Y)$ and under the chosen random string. To enable the root to generate a meaningful result, we require that the initial value, incoming messages, and coin flips fed by Alice/Bob into the oracle protocol on a node be the same as those fed into that node's oracle in the reference execution for $(X, Y)$. Furthermore, after a node has failed in the reference execution, Alice/Bob must not invoke that node's oracle any more (since that node can no longer help out). Finally, there are two special nodes $\alpha$ and $\beta$ in $G$, such that Alice and Bob will always invoke the oracle on $\alpha$ and $\beta$ (respectively) until the root generates a result. Here $\alpha$ must be the root of $G,{ }^{6}$ while $\beta$ can be any other node. During the reduction, Alice (Bob)

\footnotetext{
${ }^{6}$ This is largely for clarity, and can be relaxed if desired.
} 


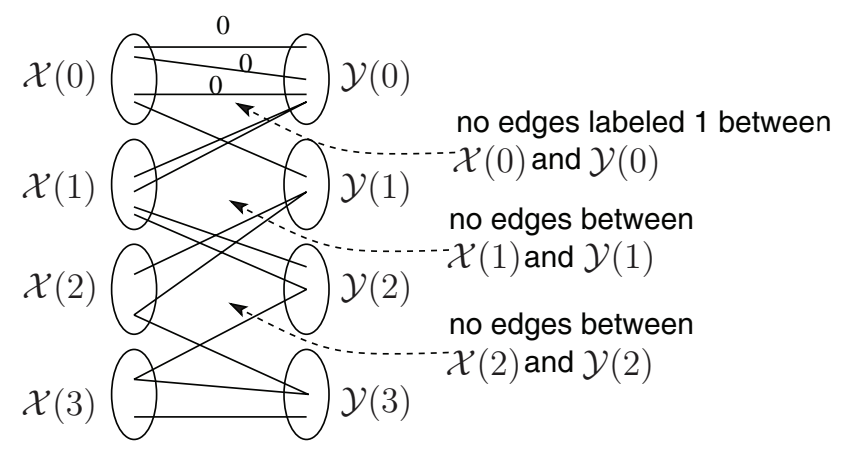

Figure 9: Example assignment graph for a given node $\tau$ and for $b^{\prime}=4 . \mathcal{X}(0), \mathcal{Y}(0), \ldots, \mathcal{X}(3)$, and $\mathcal{Y}(3)$ are the 8 subsets, which may have different sizes and different numbers of incidental edges. All edges without labels indicated have a label of 1 .

may only send to the other party all those messages sent by the oracle invocation on node $\alpha(\beta)$. This allows the establishment of a simple factor- 2 relation between the communication complexity of $\Pi$ and SUM.

Our previous reductions from UNIONSIZE and UNIONSIZECP to SUM are both oblivious reductions. Besides those two specific instances, the broad class of oblivious reductions further captures reductions from any two-party problem $\Pi$ with any promise, using any topology $G$ with any proper reference settings. We now present a strong result on the completeness of UNIONSIZECP:

Theorem 5 Consider any two-party communication complexity problem $\Pi$ that can be obliviously reduced to SUM for some topology $G$ with $N$ nodes, with the SUM oracle protocol having a time complexity of up to $b$ aggregation rounds where $b \geq 12$. For all $t \geq 1$, we have:

$$
\begin{aligned}
& \mathcal{R}_{0}^{\text {syn }}(\Pi, t) \leq \mathcal{R}_{0}^{\text {syn }}\left(\text { UnionSizeCP }_{N,\lfloor\sqrt{b / 3}\rfloor}, t\right) \\
& \mathcal{R}_{\epsilon, \delta}^{\text {syn }}(\Pi, t) \leq \mathcal{R}_{\epsilon, \delta}^{\text {syn }}\left(\text { UnionSizeCP }_{N,\lfloor\sqrt{b / 3}\rfloor}, t\right)
\end{aligned}
$$

The full proof is in Appendix $\mathrm{F}$, and we provide some intuition here. Let $\mathcal{X}$ be Alice's input domain in $\Pi$, and $\mathcal{Y}$ be Bob's. Let $\mathcal{L} \subseteq \mathcal{X} \times \mathcal{Y}$ be the set of all valid input pairs, given the promise in $\Pi$. If $\Pi$ has no promise, then $\mathcal{L}=\mathcal{X} \times \mathcal{Y}$. Given $(X, Y) \in \mathcal{L}$, an oblivious reduction has a reference setting specifying the value of each node in $G$. For any node $\tau$ where $\tau \neq \alpha$ and $\tau \neq \beta$, we define $\tau$ 's (value) assignment graph to be the bipartite graph where $\mathcal{X} \cup \mathcal{Y}$ are vertices and an edge $(X, Y)$ exists iff $(X, Y) \in \mathcal{L}$. In addition, each edge $(X, Y)$ has a binary label which is the value of $\tau$ in the reference setting for $(X, Y)$. We prove that it is always possible to partition the vertices in $\tau$ 's assignment graph into $2 b^{\prime}$ (where $b^{\prime}=\lfloor\sqrt{b / 3}\rfloor \geq 2$ ) disjoint subsets with strong properties as illustrated in Figure 9. Intuitively, this is because otherwise the reference setting for some input pair would need to have so many failures in $G$ such that $\tau$ (with a value of 1) would be disconnected from the root. Those failures are needed to ensure that Alice (Bob) can invoke the oracle on $\alpha$ $(\beta)$ throughout the execution.

At this point, we already have something close to the cycle promise - if we view each subset as a super vertex, then all the $2 b^{\prime}$ super vertices form a subgraph of a length- $2 b^{\prime}$ cycle. It is now possible to reduce $\Pi$ to UNIONSIZECP $_{N, b^{\prime}}$, by mapping an input $X$ for $\Pi$ to an input $X^{\prime}$ for UNIONSIZECP as following: Each $\tau$ in $G$ corresponds to a unique $i(1 \leq i \leq N-2)$, and $X_{i}^{\prime}$ is set to be the index of the subset in $\tau$ 's assignment graph to which $X$ belongs. Finally, $X_{N-1}^{\prime}$ is set to be the (initial) value of $\alpha$ in the given oblivious reduction, which can be obtained purely based on $X$. $X_{N}^{\prime}$ is set to be 0 . The conversion from $Y$ to $Y^{\prime}$ is similar, with $Y_{N-1}^{\prime}=0$ and $Y_{N}^{\prime}$ being the value of $\beta$. 

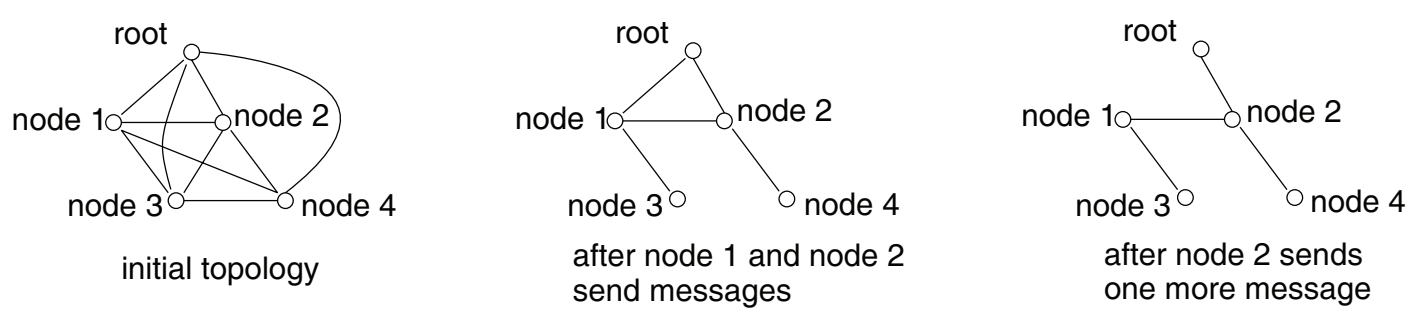

Figure 10: Example FT lower bound topology for $n=4$ and unrestricted $b$.

\section{Lower Bounds on FT Communication Complexity of SUM for All $b$}

Our previous FT lower bounds become trivial when $b>N^{0.25}$ or $\frac{1}{\epsilon^{0.5}}$. This section uses a different approach to obtain logarithmic FT lower bounds for such $b$, which is more than exponentially far away from the corresponding $O(1)$ NFT upper bounds for such $b$. We first provide some intuition under a strong gossip assumption. Later we will remove this key assumption, which is the key technical challenge addressed by our proof.

Under the gossip assumption, the root computes the sum by explicitly collecting from each node a gossip containing its value. We will show that to do so, some node will need to send $\Omega(\log N)$ messages, and hence $\Omega(\log N)$ bits even if the gossips can be fully aggregated/compressed. Here the lower bound topology will be an $N$-node clique with one of nodes being the root (Figure 10). Imagine for now that the adversary can fail edges in this topology, and further there is never more than one node sending messages in a round. These assumptions can be easily removed later once we insert some dummy nodes into each edge. Our adaptive adversary waits until exactly $\frac{N-1}{2}$ non-root nodes have sent a message (e.g., nodes 1 and 2 in Figure 10). Call these $\frac{N-1}{2}$ nodes as marked nodes. The adversary then fails enough edges so that each unmarked non-root node (e.g., node 3 ) is paired up with a marked node (e.g., node 1) and the marked node is the only gateway for the unmarked node to reach the root. Now each marked nodes has already sent a message, and yet it has one new gossip (from the corresponding unmarked node) to forward to the root. Next apply this procedure recursively on these $\frac{N-1}{2}$ marked nodes, and inject a second batch of edge failures when exactly $\frac{N-1}{4}$ of them (e.g., node 2) have sent a second message. Continuing this argument can easily show that for all the gossips to reach the root, some node needs to send at least $\log (N-1)+1$ messages.

The gossip assumption is rather strong. For example, a protocol may be such that if a node's value is 0 , then the root does not need to collect a gossip from that node and simply uses 0 as the default value. It is also possible that node $i$ sends a message to node $j$ iff node $i$ 's value is 1 , and then node $j$ conceptually relays $i$ 's value to the root, by sending a message to the root iff this value is 0 . Here the root never collects a gossip from node $i$. A key challenge in our proof is to properly capture all such possibilities. To do so, we explore a single-player probing game, and prove a strong connection between Sum protocols and strategies in this game. We then prove a lower bound on the probing game, which eventually leads to the following FT lower bounds on Sum. See Appendix G for the proof of the following theorem:

Theorem 6 For any $b \geq 1$, we have:

$$
\begin{aligned}
& \mathcal{R}_{0}^{\text {syn,ft }}\left(\operatorname{Sum}_{N}, b\right)=\Omega(\log N) \\
& \mathcal{R}_{\epsilon, \frac{1}{3}}^{\text {syn,ft }}\left(\operatorname{Sum}_{N}, b\right)=\Omega\left(\log \frac{1}{\epsilon}\right), \text { for } \epsilon=\Omega\left(\frac{1}{N}\right)
\end{aligned}
$$

\section{Discussions and Extensions}

Putting together the NFT upper bounds (Theorem 1) and FT lower bounds (Theorem 2, 3, and 6) will directly give us the exponential gaps, as summarized in Figure 1 from Section 1. Specifically, one only needs to apply 
Theorem 2 for $1 \leq b \leq 2-c$, Theorem 3 for $2-c<b \leq N^{0.25-c}$ or $\frac{1}{\epsilon^{0.5-c}}$, and Theorem 6 for $b>N^{0.25-c}$ or $\frac{1}{\epsilon^{0.5-c}}$, with $c$ being any positive constant below 0.25 . It is worth noting that such exponential gap results apply as well to the following extensions of the model defined in Section 2.

Total number of failures. Section 2 allowed the total number of failures to be up to $N-1$. In all the executions (of the SUM protocol) considered in our FT lower bound proofs, the failure adversary actually injects only $o(N)$ failures in $G$. Thus our lower bounds apply, without any modification, as long as the total number of failures is allowed to be up to any constant fraction of $N$. Our proofs carry over to even smaller number of failures, without disrupting the exponential gap, if we lower the degree of our polynomial lower bounds.

Private-coin and deterministic protocols. Section 2 only considered public-coin protocols. Private-coin protocols and deterministic protocols are also fully but implicitly covered by all our theorems. This is simply because the NFT upper bound protocol with zero-error in Theorem 1 is actually deterministic, while the one with $(\epsilon, \delta)$-error uses only private coins.

Allowing integer values for each node. In practice, each node in the network may have some integer value instead of a binary value. Our FT lower bounds obviously carry over to integer values. Our NFT upper bounds continue to apply as long as the integer value has a domain no larger than some polynomial of $N$.

Other network models. Because of the paramount practical importance of communication complexity in wireless networks, Section 2 chose to define a system model capturing wireless networks. All our theorems continue to apply regardless of whether collision is considered (i.e., whether a node can receive messages simultaneously from multiple neighbors in a round) and regardless of whether the communication is pointto-point or (local) broadcast. Note that in settings without collisions, $\Lambda(G)$ is simply the eccentricity of the root in $G$.

Letting all nodes know the result. We required only the root to learn the final result. To let all nodes know the result, the root in our upper bound protocol in Theorem 1 can simply broadcast the result to all nodes along some spanning tree.

Unknown topology. Assuming a known topology, as in Section 2, strengthens our FT lower bounds. For the upper bounds obtained via tree-aggregation, with unknown topologies, it suffices to simply add a distributed pre-processing phase for building a spanning tree.

Defining time complexity over average coin flips. Section 2 defined the time complexity of a protocol to be the number of rounds needed under the worst-case coin flips. Considering worst-case coin flips there was largely for clarity, as in the standard practice $[4,24]$ of using worst-case coin flips for defining randomized non-zero-error communication complexity. Appendix H.2 shows that defining time complexity using averagecase coin flips only affects our results slightly, and our exponential gap continues to hold.

Excluding the communication complexity of the root. Section 2 defined the communication complexity of a SUM protocol to be the number of bits sent by the bottleneck node. Here it is possible for the bottleneck node to be the root. In some scenarios, one may want to exclude the root in this definition. For example, we may be concerned with communication complexity due to the power consumption of the nodes, while the root node (e.g., a base station) may not be operating on battery power. Appendix H.1 shows that doing so does not affect any of our theorems.

\section{Conclusions and Future Work}

Tolerating crash failures has been a key focus of distributed computing research from the very beginning. Adding this fault tolerance requirement to multi-party communication complexity leads to the following natural question: "If we want to compute a function in a fault-tolerant way, what will the communication complexity be?" This paper reveals that the impact of failures on communication complexity can be large, at 
least for the SUM aggregation function in networks with general topologies. Specifically, we show that there exists (at least) an exponential gap between the NFT and FT communication complexity of SUM.

This result attests that FT communication complexity needs to be studied separately from traditional NFT communication complexity. Since this paper is only the first step along this new direction of FT communication complexity, as one would imagine, the topic is rife with interesting open questions such as:

- Our lower bound topologies for SUM are carefully constructed. We are currently investigating to what extent our lower bounds can generalize to other topologies.

- We have mainly focused on the exponential gap for SUM, and have been less concerned about specific degrees of the polynomials in the FT lower bounds. Can we further strengthen these lower bounds? Note that even our lower bound on the communication complexity of UNIONSIZECP is not tight (i.e., roughly $\frac{1}{q}$ factor from the upper bound), and thus improvement might be possible even there. Similarly, our completeness result for UNIONSIZECP is for $q=\Theta(\sqrt{b})$, while our reduction actually uses a weaker $q=\Theta(b)$.

- Our lower bounds show that the bottleneck node in $G$ will incur a large communication complexity. How many nodes in $G$ will incur asymptotically similar communication complexity as that node? Putting it another way, how many hot spots are there?

- We have defined the FT communication complexity of SUM across all protocols that can tolerate a certain number of failures. Similar to the idea of early stopping distributed consensus protocols, among this class of protocols, it would be interesting to investigate to what extent a protocol can incur a smaller communication complexity when the number of failures that actually happen (denoted as $f$ ) is small. Repeatedly invoking tree-aggregation incurs a communication complexity of $O(f \log N)$ - can we do better? We are currently investigating both upper bounds and lower bounds on this.

- Our results extend to some other functions such as SELECTION, via trivial reductions to and from SUM. But clearly there are also many interesting functions whose FT communication complexity is still unknown. In particular, can we characterize the set of functions having exponential gaps?

For answering these questions, we believe that some of the insights developed in this paper (e.g., on the role of failures in the reduction and on the cycle promise) can be valuable.

\section{Acknowledgments}

We thank Cheng Yeaw Ku and Y. C. Tay for their valuable help and pointers, and the PODC anonymous reviewers for helpful feedbacks. This work is partly supported by the research grant for the Human Sixth Sense Programme at the Advanced Digital Sciences Center from Singapore's Agency for Science, Technology and Research (A*STAR), partly supported by the research grant MOE2011-T2-2-042 "Fault-tolerant Communication Complexity in Wireless Networks" from Singapore Ministry of Education Academic Research Fund Tier-2, and partly supported by the Intel Science and Technology Center for Cloud Computing (ISTC-CC).

\section{References}

[1] N. Alon, Y. Matias, and M. Szegedy. The space complexity of approximating the frequency moments. In STOC, May 1996.

[2] O. Ayaso, D. Shah, and M. Dahleh. Information theoretic bounds for distributed computation over networks of point-to-point channels. IEEE Transactions on Information Theory, 56(12):6020-6039, 2010.

[3] T. Aysal, M. Yildiz, A. Sarwate, and A. Scaglione. Broadcast gossip algorithms for consensus. IEEE Transactions on Signal Processing, 57(7):2748-2761, July 2009. 
[4] Z. Bar-Yossef, T. S. Jayram, R. Kumar, and D. Sivakumar. An information statistics approach to data stream and communication complexity. Journal of Computer and System Sciences, 68(4):702-732, June 2004.

[5] M. Bawa, A. Gionis, H. Garcia-Molina, and R. Motwani. The price of validity in dynamic networks. Journal of Computer and System Sciences, 73(3):245-264, May 2007.

[6] A. Blokhuis. On the sperner capacity of the cyclic triangle. Journal of Algebraic Combinatorics, 2(2):123-124, June 1993.

[7] S. Boyd, A. Ghosh, B. Prabhakar, and D. Shah. Randomized gossip algorithms. IEEE Transactions on Information Theory, 52(6):2508-2530, June 2006.

[8] M. Braverman and A. Rao. Towards coding for maximum errors in interactive communication. In STOC, June 2011.

[9] A. Chakrabarti and O. Regev. An optimal lower bound on the communication complexity of gap-hammingdistance. In STOC, June 2011.

[10] A. Chandra, M. Furst, and R. Lipton. Multi-party protocols. In STOC, April 1983.

[11] J. Chen and G. Pandurangan. Optimal gossip-based aggregate computation. In SPAA, June 2010.

[12] J. Chen, G. Pandurangan, and D. Xu. Robust computation of aggregates in wireless sensor networks: Distributed randomized algorithms and analysis. In IPSN, April 2005.

[13] J. Considine, F. Li, G. Kollios, and J. Byers. Approximate aggregation techniques for sensor databases. In ICDE, March 2004.

[14] A. Dhulipala, C. Fragouli, and A. Orlitsky. Silence-based communication. IEEE Transactions on Information Theory, 56(1):350-366, January 2010.

[15] I. Eyal, I. Keidar, and R. Rom. LiMoSense - Live Monitoring in Dynamic Sensor Networks. In ALGOSENSORS, September 2011.

[16] P. Flajolet and G. N. Martin. Probabilistic counting algorithms for data base applications. Journal of Computer and System Sciences, 31(2):182-209, September 1985.

[17] A. Giridhar and P. R. Kumar. Towards a theory of in-network computation in wireless sensor networks. IEEE Communications Magazine, 44(4):98-107, April 2006.

[18] P. Gupta and P. R. Kumar. The capacity of wireless networks. IEEE Transactions on Information Theory, 46(2):388-404, March 2000.

[19] R. Impagliazzo and R. Williams. Communication complexity with synchronized clocks. In CCC, June 2010.

[20] M. Jelasity, A. Montresor, and O. Babaoglu. Gossip-based aggregation in large dynamic networks. ACM Transactions on Computer Systems, 23(3):219-252, August 2005.

[21] P. Jesus, C. Baquero, and P. Almeida. Fault-tolerant aggregation by flow updating. In DAIS, June 2009.

[22] S. Kashyap, S. Deb, K. Naidu, R. Rastogi, and A. Srinivasan. Efficient gossip-based aggregate computation. In PODS, June 2006.

[23] D. Kempe, A. Dobra, and J. Gehrke. Gossip-based computation of aggregate information. In FOCS, October 2003.

[24] E. Kushilevitz and N. Nisan. Communication Complexity. Cambridge University Press, 1996.

[25] D. Mosk-Aoyama and D. Shah. Computing separable functions via gossip. In PODC, July 2006.

[26] S. Nath, P. Gibbons, S. Seshany, and Z. Anderson. Synopsis diffusion for robust aggregation in sensor networks. ACM Transactions on Sensor Networks, 4(2), March 2008.

[27] I. Newman. Private vs. common random bits in communication complexity. Information Processing Letters, 39(2):67-71, July 1991.

[28] S. Rajagopalan and L. Schulman. A coding theorem for distributed computation. In STOC, May 1994. 
[29] L. Schulman. Coding for interactive communication. IEEE Transactions on Information Theory, 42(6):17451756, 1996.

[30] D. Woodruff. Optimal space lower bounds for all frequency moments. In SODA, January 2004.

[31] H. Yu. Secure and highly-available aggregation queries in large-scale sensor networks via set sampling. Distributed Computing, 23(5):373-394, April 2011. 


\section{A Some Useful Known Results}

This section describes some known results that this paper uses. (This is the only such section in this paper.) These results and their proofs are not our contribution. We include the details and sometime the proofs here only for completeness, because some of them were folklore results, or were not formally stated, or were not stated to cover FT communication complexity, or were proved under slightly different models in a restricted form. In the next, the notations $\mathcal{R}_{0, \delta}, \mathcal{R}_{0, \delta}^{\text {syn }}$, and $\mathcal{R}_{0, \delta}^{\text {syn, ft }}$ simply mean $\mathcal{R}_{\epsilon, \delta}, \mathcal{R}_{\epsilon, \delta}^{\text {syn }}$, and $\mathcal{R}_{\epsilon, \delta}^{\text {syn,ft }}$ with $\epsilon=0$, respectively.

Folklore fault-tolerant protocol for computing MAX. The following sketches a simple folklore faulttolerant protocol for efficiently computing MAX. This protocol is a simplified version of some more complex protocols in the literature (e.g., the protocol from [31] which tolerates byzantine failures). Same as for SuM, each node here has an integer value whose domain is no larger than some polynomial of $N$, where $N$ is the total number of nodes in the network. This protocol does a simple binary search on the entire value domain. At each step of the binary search, the protocol conceptually asks whether any node in the system has a value that is no smaller than some specific value. Clearly, we only need to ask $O(\log N)$ such questions to determine MAX. For each such question, a node floods a single bit of "1" (without including its id) as the reply if its value is no smaller than the specified value. Other nodes in the system will relay/forward only the very first reply they see for that question (this is a simplified version of the keyed predicate test protocol in [31] which tolerates byzantine failures). One can easily show that this process is fault-tolerant, and each question only requires each node to send $O(1)$ bits. Furthermore, at the end of the flooding, each node in the system will know the answer to this question, and thus can infer what the next question will be. Thus posing the questions does not incur any extra communication. The total number of bits each node needs to send in this fault-tolerant protocol for MAX is $O(\log N)$.

Known relation between $\mathcal{R}_{0}, \mathcal{R}_{0}^{\mathrm{syn}, \mathrm{ft}}$ and $\mathcal{R}_{0, \delta}, \mathcal{R}_{0, \delta}^{\mathrm{syn}, \mathrm{ft}}$. Note that we do not necessarily have $\mathcal{R}_{0} \geq \mathcal{R}_{0, \delta}$, since $\mathcal{R}_{0}$ is the average-case (over random coin flips in the protocol) communication complexity, while $\mathcal{R}_{0, \delta}$ is the worst-case (over random-coin flips in the protocol) communication complexity. Nevertheless, the following relation in Lemma 7 is well-known [24]. This relation trivially applies to fault-tolerant communication complexity as well.

Lemma 7 (Adapted from [24].) For any communication complexity problem $\Pi$ and $\delta>0, \mathcal{R}_{0}(\Pi) \geq$ $\delta \mathcal{R}_{0, \delta}(\Pi)$. Similarly for any $b \geq 1$ and $\delta>0, \mathcal{R}_{0}^{\mathrm{syn}, \mathrm{ft}}\left(\operatorname{Sum}_{N}, b\right) \geq \delta \mathcal{R}_{0, \delta}^{\mathrm{syn}, \mathrm{ft}}\left(\mathrm{SuM}_{N}, b\right)$.

Proof: Consider the optimal zero-error randomized protocol for $\Pi$, which generates a zero-error result while incurring an expected (over the random coin flips in the protocol) communication complexity of $\mathcal{R}_{0}(\Pi)$ bits. By Markov's inequality, the protocol's communication complexity exceeds $\mathcal{R}_{0}(\Pi) / \delta$ bits with probability at most $\delta$. We can thus construct a new protocol which behaves the same as the original one except that a node stops once it has sent $\mathcal{R}_{0}(\Pi) / \delta$ bits. Obviously, this protocol outputs correct results with probability at least $1-\delta$, and incurs a worst-case communication complexity of $\mathcal{R}_{0}(\Pi) / \delta$ bits, implying $\mathcal{R}_{0, \delta}(\Pi) \leq \mathcal{R}_{0}(\Pi) / \delta$. A similar proof can show $\mathcal{R}_{0, \delta}^{\mathrm{syn}, \mathrm{ft}}\left(\mathrm{SuM}_{N}, b\right) \leq \mathcal{R}_{0}^{\mathrm{syn}, \mathrm{ft}}\left(\mathrm{SuM}_{N}, b\right) / \delta$.

Known relation between $\mathcal{R}_{0}, \mathcal{R}_{\epsilon, \delta}$ and $\mathcal{R}_{0}^{\mathrm{syn}}, \mathcal{R}_{\epsilon, \delta}^{\mathrm{syn}}$. The following lemma is a slightly extended version of the corresponding theorem from [19], which draws a connection between NFT communication complexity with synchronized rounds and NFT communication complexity without synchronized rounds. Since our synchronous round model is slightly different from [19], we provide a proof sketch below for the sake of completeness. This proof is not our contribution.

Lemma 8 (Adapted from [19].) For any two-party communication complexity problem $\Pi$ and any $t \geq 2$, we have $\mathcal{R}_{0}(\Pi)=\mathcal{R}_{0}^{\mathrm{syn}}(\Pi, t) \cdot O(\log t)$ and $\mathcal{R}_{\epsilon, \delta}(\Pi)=\mathcal{R}_{\epsilon, \delta}^{\mathrm{syn}}(\Pi, t) \cdot O(\log t)$. 
Proof: Consider any given protocol $\mathcal{P}$ (with $\mathcal{P}_{A}$ being Alice's part of the protocol and $\mathcal{P}_{B}$ being Bob's part), that can solve $\Pi$ under the synchronous round setting with $a$ bits (either on expectation or worst-case) of communication, while always terminating within $t$ synchronous rounds. We construct a protocol $\mathcal{Q}$ (with $\mathcal{Q}_{A}$ and $\mathcal{Q}_{B}$ similarly defined) that can solve the problem with $O(a \log t)$ bits (either on expectation or worst-case, respectively) of communication complexity in the classic setting without synchronous rounds.

In $\mathcal{Q}$, Alice and Bob each maintains a local counter initialized to 1 . These two counters correspond to the round number needed by $\mathcal{P}$. Let the current counter value on Alice be $r_{A}$. In $\mathcal{Q}_{A}$, Alice first tries executing $\mathcal{P}_{A}$ for rounds $r_{A}, r_{A}+1, r_{A}+2, \ldots$, while assuming that $\mathcal{P}_{B}$ does not send any message in any of those rounds. Alice then determines $r_{A}^{\prime}\left(r_{A}^{\prime} \geq r_{A}\right)$, the first round during which $\mathcal{P}_{A}$ sends a message in this trial execution. Similarly Bob determines $r_{B}^{\prime}$. Alice and Bob then exchange $r_{A}^{\prime}$ and $r_{B}^{\prime}$, taking $2 \log t$ bits. Let $r^{\prime}=\min \left(r_{A}^{\prime}, r_{B}^{\prime}\right)$. Alice next executes $\mathcal{P}_{A}$ (for real) for rounds $r_{A}, r_{A}+1, \ldots, r^{\prime}$, and then sends a message to Bob if $r_{A}^{\prime}=r^{\prime}$. Similarly in $\mathcal{Q}_{B}$, Bob executes $\mathcal{P}_{B}$ for rounds $r_{B}, r_{B}+1, \ldots, r^{\prime}$, and then sends a message to Alice if $r_{B}^{\prime}=r^{\prime}$. Note that for round $r^{\prime}, \mathcal{P}$ must incur at least one bit of communication. Thus for each bit $\mathcal{P}$ incurs, $\mathcal{Q}$ incurs at most $2 \log t+1=O(\log t)$ bits. After the message exchange for round $r^{\prime}$, Alice and Bob set $r_{A}=r^{\prime}+1$ and $r_{B}=r^{\prime}+1$, and repeat the above process until $\mathcal{P}$ terminates.

Known results on UNIONSIZE's communication complexity. Trivially combining several recent results [9, $19,30]$ leads to the following known results:

Theorem 9 (Adapted from [9].) $\mathcal{R}_{0}\left(\mathrm{UNIONSIZE}_{n}\right)=\Omega(n)$ and $\mathcal{R}_{\epsilon, \frac{1}{3}}\left(\mathrm{UNIONSIZE}_{n}\right)=\Omega\left(\frac{1}{\epsilon^{2}}\right)$ for $\epsilon=$ $\Omega\left(\frac{1}{\sqrt{n}}\right)$.

Proof: We first prove the theorem for $\epsilon \geq \frac{1}{\sqrt{n}}$. This proof for $\mathcal{R}_{\epsilon, \frac{1}{3}}\left(\mathrm{UNIONSIZE}_{n}\right)=\Omega\left(\frac{1}{\epsilon^{2}}\right)$ trivially plugs in a recent strong result [9] on the GHD (Gap-Hamming-Distance) problem into an existing reduction [30] from GHD to UnIONSize. Consider any given $\epsilon>0$. In GHD $\frac{2}{5 \epsilon^{2}}$, Alice and Bob have binary strings $X$ and $Y$ as inputs respectively, where each string has $\frac{2}{5 \epsilon^{2}}$ bits. Let $\Delta(X, Y)$ denote the hamming distance between $X$ and $Y$. Alice and Bob are further given the promise that either $\Delta(X, Y)>\frac{1}{5 \epsilon^{2}}+\frac{1}{\epsilon}$ or $\Delta(X, Y) \leq \frac{1}{5 \epsilon^{2}}-\frac{1}{\epsilon}$. They should output 1 iff $\Delta(X, Y)$ satisfies the first inequality. It is proved recently by Chakrabarti and Regev [9] that $\mathcal{R}_{0, \frac{1}{3}}\left(\mathrm{GHD}_{\frac{2}{5 \epsilon^{2}}}\right)=\Omega\left(\frac{1}{\epsilon^{2}}\right)$.

To reduce GHD to UnIONSIZE, given input string $X$ for $\mathrm{GHD}_{\frac{2}{5 \epsilon^{2}}}$, Alice locally generates an input $X^{\prime}$ for UNIONSIZE $n$ by appending 0 to the length- $\frac{2}{5 \epsilon^{2}}$ binary string $X$ until the length reaches $n$. Bob similarly generates $Y^{\prime}$. Let $\left|X^{\prime}\right|$ and $\left|Y^{\prime}\right|$ denote the hamming weight of $X^{\prime}$ and $Y^{\prime}$, respectively. Thus we have $\operatorname{UnIONSIZE}\left(X^{\prime}, Y^{\prime}\right)=\left(\left|X^{\prime}\right|+\left|Y^{\prime}\right|+\Delta\left(X^{\prime}, Y^{\prime}\right)\right) / 2=(|X|+|Y|+\Delta(X, Y)) / 2$. Leveraging the promise on $X$ and $Y$, we have:

- If $\Delta(X, Y)>\frac{1}{5 \epsilon^{2}}+\frac{1}{\epsilon}$, then $\operatorname{UniOnSiZE}\left(X^{\prime}, Y^{\prime}\right)>\left(|X|+|Y|+\frac{1}{5 \epsilon^{2}}+\frac{1}{\epsilon}\right) / 2$.

- If $\Delta(X, Y) \leq \frac{1}{5 \epsilon^{2}}-\frac{1}{\epsilon}$, then $\operatorname{UniONSIZE}\left(X^{\prime}, Y^{\prime}\right) \leq\left(|X|+|Y|+\frac{1}{5 \epsilon^{2}}-\frac{1}{\epsilon}\right) / 2$.

One can easily verify that for all $\epsilon$, we have $(1+\epsilon)\left(|X|+|Y|+\frac{1}{5 \epsilon^{2}}-\frac{1}{\epsilon}\right) / 2<(1-\epsilon)\left(|X|+|Y|+\frac{1}{5 \epsilon^{2}}+\frac{1}{\epsilon}\right) / 2$. Next Bob tells Alice the size of $Y$, using $\log |Y|=O\left(\log \frac{1}{\epsilon}\right)$ bits. Alice can now pick any value between the above two values as the threshold. Alice outputs 1 iff the $\operatorname{UnIONS\operatorname {Siz}}\left(X^{\prime}, Y^{\prime}\right)$ execution returns a value above the threshold. Finally, Alice informs Bob of the result, using a single bit. (This is needed since GHD requires both Alice and Bob to know the result.) Since $\mathcal{R}_{0, \frac{1}{3}}\left(\mathrm{GHD}_{\frac{2}{5 \epsilon^{2}}}\right)=\Omega\left(\frac{1}{\epsilon^{2}}\right)$, we have $\mathcal{R}_{\epsilon, \frac{1}{3}}\left(\mathrm{UNIONSIZE}_{n}\right)=$ $\Omega\left(\frac{1}{\epsilon^{2}}-\log \frac{1}{\epsilon}-1\right)=\Omega\left(\frac{1}{\epsilon^{2}}\right)$. Now by Lemma 7, we have:

$$
\mathcal{R}_{0}\left(\operatorname{UNIONSIZE}_{n}\right) \geq \frac{1}{3} \mathcal{R}_{0, \frac{1}{3}}\left(\operatorname{UNIONSIZE}_{n}\right) \geq \frac{1}{3} \mathcal{R}_{\frac{1}{\sqrt{n}}, \frac{1}{3}}\left(\operatorname{UNIONSIZE}_{n}\right)=\Omega(n)
$$


Finally, we still need to cover the case for $\epsilon=\Omega\left(\frac{1}{\sqrt{n}}\right)$ but $\epsilon<\frac{1}{\sqrt{n}}$. For such $\epsilon$ (which is necessarily $\Theta\left(\frac{1}{\sqrt{n}}\right)$ ), we have:

$$
\mathcal{R}_{\epsilon, \frac{1}{3}}\left(\operatorname{UNIONSIZE}_{n}\right) \geq \mathcal{R}_{\frac{1}{\sqrt{n}}, \frac{1}{3}}\left(\operatorname{UNIONSIZE}_{n}\right)=\Omega(n)=\Omega\left(\frac{1}{\epsilon^{2}}\right)
$$

Combining Theorem 9 and Lemma 8 gives:

Corollary 10 Let $\operatorname{poly}(n)$ be any polynomial of $n$ with constant degree, we have $\mathcal{R}_{0}^{\mathrm{Syn}}$ (UNIONSIZE $n$, $O(\operatorname{poly}(n)))=\Omega\left(\frac{n}{\log n}\right)$ and $\mathcal{R}_{\epsilon, \frac{1}{3}}^{\mathrm{syn}}\left(\mathrm{UNIONSIZE}_{n}, O(\operatorname{poly}(n))\right)=\Omega\left(\frac{1}{\epsilon^{2} \log n}\right)$ for $\epsilon=\Omega\left(\frac{1}{\sqrt{n}}\right)$.

\section{B Tree-Aggregation Protocol with $O\left(\log \frac{1}{\epsilon}+\log \log N\right)$ Aggregation Message Size}

This section provides the details of the tree-aggregation protocol with only $O\left(\log \frac{1}{\epsilon}+\log \log N\right)$ aggregation message size, as mentioned in Section 3.

Protocol intuition. First, we should note that directly encoding each partial sum with $O\left(\log \frac{1}{\epsilon}+\log \log N\right)$ bits using a floating-point-style representation will not actually work, due to underflow issues when sequentially adding many small numbers to a large number. Thus instead, we will apply a similar trick as AMS synopsis [1]. Intuitively in this protocol, each "1" value in the system is flagged with a certain probability. The system then uses the simple tree-aggregation protocol from Section 3 to determine the exact total number (sum) of such flagged "1" values. By properly adjusting the flagging probability, we can always ensure that this sum is no larger than $120 / \epsilon^{2}$, and thus the size of the aggregation message will be no larger than $\log \left(120 / \epsilon^{2}\right)$. Furthermore, it is possible to dynamically adjust such flagging probability in one pass of the aggregation protocol, without any global coordination. Finally, the root estimates the final result for SUM based on the sum of flagged " 1 " values and the associated flagging probability.
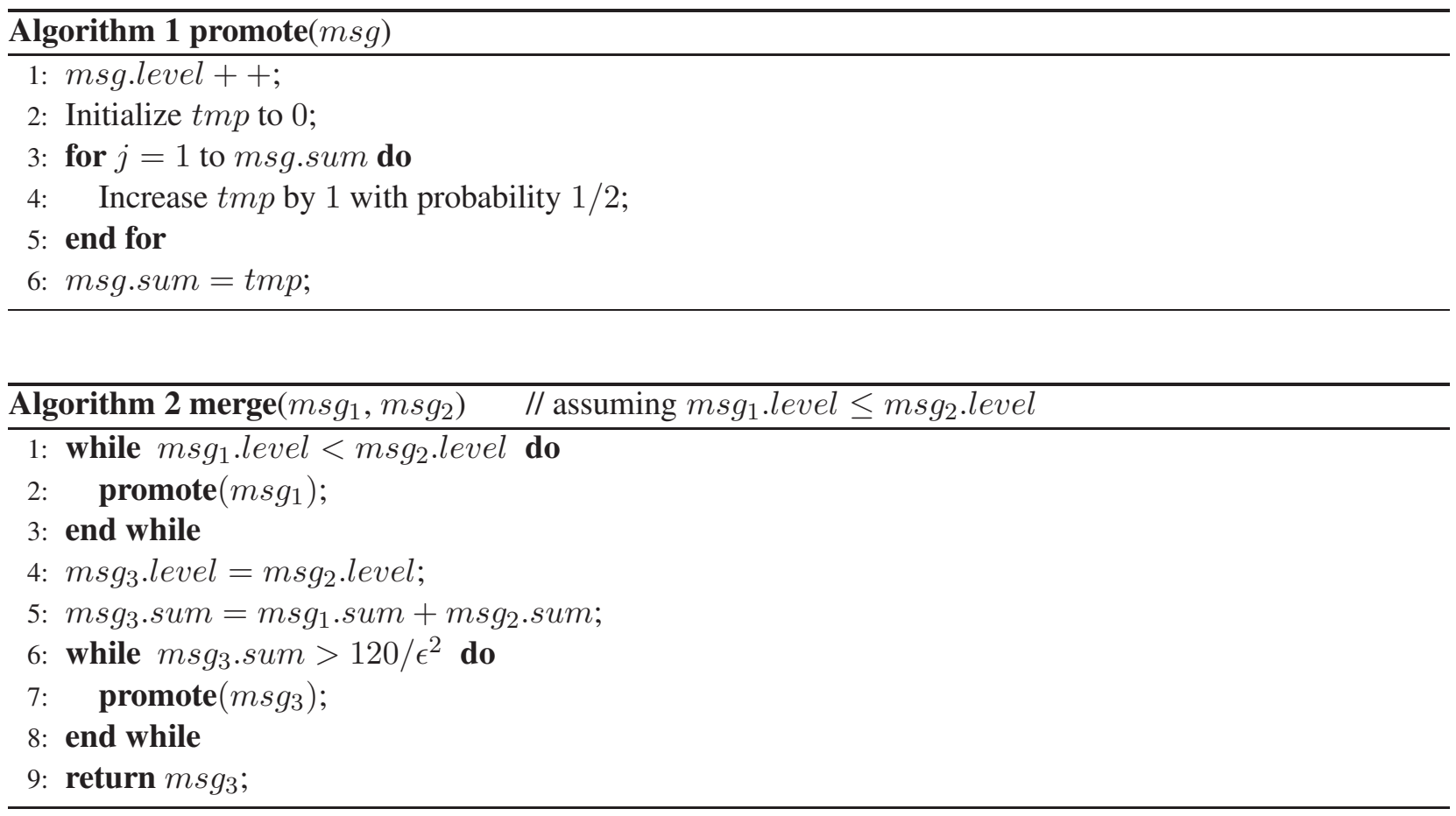
Protocol description and pseudo-code. Specifically in this protocol, each aggregation message contains an integer sum $\in\left[0,120 / \epsilon^{2}\right]$ and an integer level $\in[0, \log N]$. Intuitively, these two integers mean that if each " 1 " value in the subtree is flagged with probability $2^{- \text {level }}$, then the partial sum of the flagged values is sum. A node with a value of 1 generates an aggregation message with $\mathrm{sum}=1$ and level $=0$, for its own value. Intermediate tree nodes will need to combine multiple aggregation messages into one. Without loss of generality, we only need to explain how to combine two aggregation messages $m s g_{1}$ and $m s g_{2}$ into one, where $m s g_{1}$.level $\leq m s g_{2}$.level. We promote (Algorithm 1) an aggregation message $m s g_{1}$, by i) increasing $m s g_{1}$.level by one, and ii) tossing $\mathrm{msg}_{1} . \mathrm{sum}$ fair coins and then updating $\mathrm{msg}_{1} . \mathrm{sum}$ to be the total number of heads we observe. To merge $m s g_{1}$ and $m s g_{2}$ into $m s g_{3}$ (Algorithm 2), we first repeatedly promote $\mathrm{msg}_{1}$, until $\mathrm{msg}_{1}$. level $=\mathrm{msg}_{2}$. level. We then set $\mathrm{msg}_{3} . l e v e l=\mathrm{msg}_{2} . l e v e l$, and $\mathrm{msg}_{3} . \mathrm{sum}=$ $m s g_{1} . s u m+m s g_{2} . s u m$. If $m s g_{3} . s u m>120 / \epsilon^{2}$, we will again repeatedly promote $\mathrm{msg}_{3}$ until the first time that $\mathrm{msg}_{3} . s u m \leq 120 / \epsilon^{2}$. Finally, imagine that the root has a virtual parent and let $\mathrm{msg}$ be the aggregation message sent by the root to its virtual parent. The root will estimate the final sum to be msg.sum $\times 2^{\text {msg.level }}$.

Formal properties. It is obvious that the number of bits sent by each node in this protocol is $O\left(\log \frac{1}{\epsilon}+\right.$ $\log \log N)$. We next prove that the protocol does give us an $(\epsilon, 1 / 3)$-approximate result:

Theorem 11 Consider any graph $G$ with $N$ nodes and any constant $\epsilon \in(0,1]$. If we denote $s$ as the exact sum of the values of all the $N$ nodes and $\hat{s}$ as the estimated sum output by the above protocol, then

$$
\operatorname{Pr}[(1-\epsilon) s \leq \hat{s} \leq(1+\epsilon) s] \geq \frac{2}{3}
$$

Proof: Consider the sequence of random variables $S_{0}, S_{1}, \ldots$, where $S_{0}=s$ and $S_{i+1}$ (for $i \geq 0$ ) is the number of heads observed when flipping a fair coin exactly $S_{i}$ times. Furthermore, for generating $S_{i+1}$, the random process uses the same coin flip results as the protocol uses in promoting all messages with level $=i$ (i.e., at Line 4 of Algorithm 1). Let random variable $L$ be the smallest integer such that $S_{L} \leq z$ where $z=\frac{120}{\epsilon^{2}}$. Let $m s g$ be the aggregation message sent by the root to its virtual parent. We claim that msg.level $=L$ and msg.sum $=S_{L}$. First, it is impossible for msg.level $<L$, since otherwise msg.sum will be above $z$ and thus the $m s g$ will be promoted by the root. Next if msg.level $>L$, it means that some node must have observed a message $m s g^{\prime}$ whose level is $L$, and has further promoted $m s g^{\prime}$. But this is impossible since if $m s g^{\prime}$.level $=L$, then $m s g^{\prime}$.sum $\leq S_{L} \leq z$ by our definition of $L$. Now given that $m s g$.level $=L$, we have msg.sum $=S_{L}$.

Let $l=\left\lfloor\log _{2} \frac{3 s}{4 z}\right\rfloor$, and we have $2^{l} \in\left[\frac{3 s}{8 z}, \frac{3 s}{4 z}\right]$ and $2^{l+2} \in\left[\frac{3 s}{2 z}, \frac{3 s}{z}\right]$. Since for all $i \geq 0, S_{i}$ is a binomial random variable with parameter $\left(s, 2^{-i}\right)$, we have

$$
\begin{aligned}
& \mathrm{E}\left[S_{l}\right]=2^{-l} s \geq \frac{4}{3} z \quad \text { and } \quad \operatorname{VAR}\left[S_{l}\right] \leq \frac{8}{3} z \\
& \mathrm{E}\left[S_{l+2}\right]=2^{-l-2} s \leq \frac{2}{3} z \quad \text { and } \quad \operatorname{VAR}\left[S_{l+2}\right] \leq \frac{2}{3} z
\end{aligned}
$$

We claim that with probability at most $\frac{1}{4}, L \notin[l+1, l+2]$, since by Chebyshev's inequality:

$$
\begin{aligned}
\operatorname{Pr}[L \leq l] & =\operatorname{Pr}\left[S_{l} \leq z\right] \leq \frac{24}{z} \leq \frac{1}{5} \\
\operatorname{Pr}[L>l+2] & =\operatorname{Pr}\left[S_{l+2}>z\right] \leq \frac{6}{z} \leq \frac{1}{20}
\end{aligned}
$$

Denote $\mathcal{E}_{i}$ as the event $2^{i} S_{i} \notin[(1-\epsilon) s,(1+\epsilon) s]$, and we claim that for any $i \leq l+2, \operatorname{Pr}\left[\mathcal{E}_{i}\right] \leq \frac{1}{40}$. Since $S_{i}$ is a binomial random variable with parameter $\left(s, 2^{-i}\right)$, We have $\mathrm{E}\left[2^{i} S_{i}\right]=s$ and $\operatorname{VAR}\left[2^{i} S_{i}\right] \leq 2^{2 i} 2^{-i} s=2^{i} s$. By Chebyshev's inequality, we have $\operatorname{Pr}\left[\mathcal{E}_{i}\right]=1-\operatorname{Pr}\left[2^{i} S_{i} \in[(1-\epsilon) s,(1+\epsilon) s]\right] \leq \frac{2^{i}}{\epsilon^{2} s} \leq \frac{3}{z \epsilon^{2}}=\frac{1}{40}$. Next, 


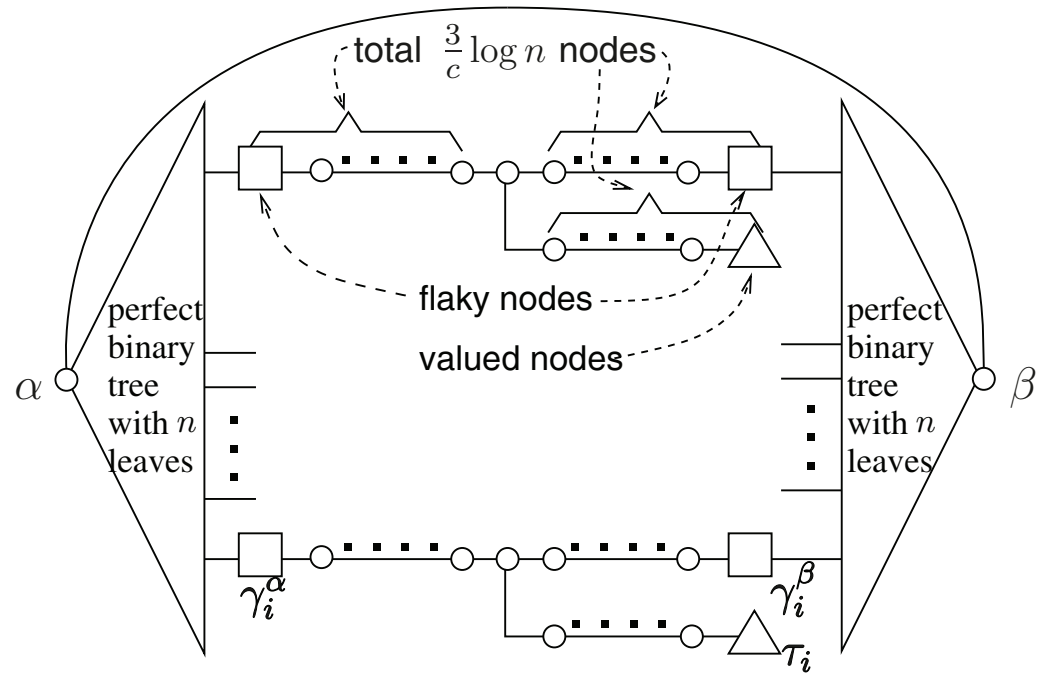

Figure 11: FT lower bound topology for $b \leq 2-c$.

denote $\mathcal{E}$ as the event that $\hat{s} \notin[(1-\epsilon) s,(1+\epsilon) s]$, or equivalently $2^{L} S_{L} \notin[(1-\epsilon) s,(1+\epsilon) s]$. We have:

$$
\begin{aligned}
\operatorname{Pr}[\mathcal{E}] & =\sum_{i} \operatorname{Pr}[L=i] \operatorname{Pr}[\mathcal{E} \mid L=i] \\
& =\sum_{i \in[l+1, l+2]} \operatorname{Pr}[L=i] \operatorname{Pr}[\mathcal{E} \mid L=i]+\sum_{i \notin[l+1, l+2]} \operatorname{Pr}[L=i] \operatorname{Pr}[\mathcal{E} \mid L=i] \\
& \leq \operatorname{Pr}[L=l+1] \operatorname{Pr}\left[\mathcal{E}_{l+1} \mid L=l+1\right]+\operatorname{Pr}[L=l+2] \operatorname{Pr}\left[\mathcal{E}_{l+2} \mid L=l+2\right]+\sum_{i \notin[l+1, l+2]} \operatorname{Pr}[L=i] \\
& \leq \operatorname{Pr}\left[\mathcal{E}_{l+1} \text { and } L=l+1\right]+\operatorname{Pr}\left[\mathcal{E}_{l+2} \text { and } L=l+2\right]+\frac{1}{4} \\
& \leq \operatorname{Pr}\left[\mathcal{E}_{l+1}\right]+\operatorname{Pr}\left[\mathcal{E}_{l+2}\right]+\frac{1}{4} \leq \frac{1}{20}+\frac{1}{4}<\frac{1}{3}
\end{aligned}
$$

\section{Omitted Details from Section 4}

\section{C.1 A Slightly Improved Construction}

The topology in Section 4 (Figure 2) works for $1 \leq b \leq \frac{10}{9}$. To obtain the desired FT lower bound for $1 \leq b \leq 2-c$ (where $c$ is any positive constant) in Theorem 2, we use the improved topology in Figure 11 . The $n$ chains in the topology in Figure 2 are now replaced with $n T$-structures, where $n$ is still a power of 2 . The $i$ th T-structure has 3 sequences of $\frac{3}{c} \log n$ nodes (total $\frac{9}{c} \log n$ nodes) attached to a degree- 3 node in the middle. For the first sequence, the last node at the other end is a valued node $\tau_{i}$. Let $\gamma_{i}^{\alpha}$ and $\gamma_{i}^{\beta}$ denote the last node at the other end of each of the remaining two sequences, respectively. Both of them are flaky nodes. Same as in Figure 2, we next construct a perfect binary tree with all the $\gamma_{i}^{\alpha}$ 's being the leaves, and let node $\alpha$ denote the tree root. Similarly construct a second perfect binary tree whose leaves are all the $\gamma_{i}^{\beta}$ 's, and let node $\beta$ denote the tree root. Finally, we connect $\alpha$ with $\beta$ using a single edge, and let $\alpha$ be the root of the topology. It is easy to verify that there are $N=\frac{9}{c} n \log n+3 n-2$ nodes in the topology. The valued node 
$\tau_{i}$ has a binary value of 1 iff $X_{i} \neq 0$ or $Y_{i} \neq 0$. If $X_{i}=0$, then the flaky node $\gamma_{i}^{\alpha}$ fails at the beginning of round $t_{0}=\frac{6}{c} \log n+1$. Otherwise it never fails. Similarly, $\gamma_{i}^{\beta}$ fails at the beginning of round $t_{0}$ iff $Y_{i}=0$.

\section{C.2 A Formal Framework for Reasoning about Reductions to SUM}

To prepare for our formal reasoning next, we need to develop a simple formal framework. Since FT communication complexity has not been formally studied before, many of the concepts in this framework need to be defined from scratch. This formal framework will also be used later for the proofs in Appendix D and Appendix F.3. For that reason, the framework developed here will be more general than what is needed for proving the results in Section 4.

Rounds and failures. The execution of the SUM oracle protocol starts at round 1. We sometimes for convenience also discuss round 0 , during which the SuM protocol does nothing. Note that one can assume, without loss of generality, that all failures happen at the beginning of various rounds: ${ }^{7}$ If a node $v$ fails sometime within round $r$, since $v$ can (locally) broadcast at most one message in a round, the failure can be viewed as happening at the beginning of round $r+1$ if the failure occurs after $v$ sends the message. Otherwise the failure can be viewed as happening at the beginning of round $r .{ }^{8}$ Thus from now on, we will assume that all failures happen at the beginning of various rounds. If a node fails at the beginning of round $r$, we say that the failure time of that node is round $r$.

Simulating a node. To properly simulate a node (i.e., simulate the execution of the SUM oracle protocol on that node) in a certain round, Alice (Bob) needs to feed all necessary parameters to the oracle protocol running on that node. The execution of a randomized oracle protocol on a node in a given round is uniquely determined by the (public) coin flips, the topology (since the topology is known), the id of the node, the (initial) value of the node, the failure time of the node (i.e., a failed node should not send out messages and the oracle protocol should not be invoked on such a node), and all the incoming messages to this node since round 1. Alice can easily generate the coin flips, and she already knows the topology and node id. For some nodes, Alice can uniquely determine their values and failure time based on Alice's input $X$. Finally, the incoming messages to a node $v$ will have to be obtained via Alice's simulation of $v$ 's neighbors or directly from Bob if $\beta$ is the sender of that message. Recall that in a round, a node first performs some local computation, and then does either a send or a receive. In particular, the potential message received by a node can only affect its behavior starting from the next round, since the node does not do any further local computation in the current round after the receive operation. Thus regardless of whether $v$ does a send or receive in round $r$, to simulate $v$ in round $r$, we (only) need all the incoming messages to $v$ from round 1 to round $r-1$ (inclusive).

Epicenters and their occurrence time. The following concepts are always defined with respect to a given input $X$ of Alice's. A node $v$ in $G$ is a value epicenter if $v$ 's value is not uniquely determined by $X$. Namely given $X$, there exists Bob's inputs $Y$ and $Y^{\prime}$ such that $v$ 's value is different under the simulated execution of the Sum oracle protocol (used in the reduction) for $(X, Y)$ and $\left(X, Y^{\prime}\right)$. A node $v$ is a failure epicenter if $v$ is not already a value epicenter and if $v$ 's failure time is not uniquely determined by $X$. Value epicenters and failure epicenter are all called epicenters.

The occurrence time of a value epicenter $v$ is defined to be round 1 . The occurrence time of a failure epicenter $v$ is defined to be $v$ 's earliest failure time, across all valid $Y$ 's given the current $X$. To get some intuition behind the occurrence time, consider a failure epicenter $v$. Suppose that given $X$, the only possible inputs to Bob are $Y$ and $Y^{\prime}$. Imagine that $v$ fails at the beginning of round 3 if Bob's input is $Y$, and fails at the beginning of round 8 if Bob's input is $Y^{\prime}$. Since Alice does not know Bob's input, starting from round 3,

\footnotetext{
${ }^{7}$ In other words, by injecting failures only at the beginning of various rounds, our failure adversary in Section 4 has already fully utilized the flexibility on failure time.

${ }^{8}$ For wired network settings with point-to-point communication, this assumption will no longer be without of loss of generality. While all our final results will still hold without any modification, the formal framework needs to be slightly different.
} 
Alice no longer knows whether $v$ is still alive and thus can no longer simulate $v$. This also explains why a value epicenter $v$ has an occurrence time of round 1 - Alice cannot simulate $v$ even for round 1 .

Spoil paths and spoiled nodes. All the following concepts are still with respect to a given input $X$ of Alice's. If a node's failure time $r$ is uniquely determined by $X$, we say that the node fails stably at the beginning of round $r$. We also call such a failure a stable failure. A spoil path from an epicenter $u_{0}$ (occurring at round $r_{0}$ ) to a node $v$ is a sequence of nodes $u_{0}, u_{1}, u_{2}, \ldots, u_{k}, v$ where

- for $0 \leq i \leq k, u_{i} \neq \alpha$ and $u_{i} \neq \beta$,

- $v$ is $u_{k}$ 's neighbor and $u_{i}$ is $u_{i-1}$ 's neighbor for $1 \leq i \leq k$,

- $v$ has not failed stably before the beginning of round $r_{0}+k+2$, and $u_{i}$ has not failed stably before the beginning of round $r_{0}+i+1$ for $0 \leq i \leq k$. Intuitively, this enables $u_{i}$ to potentially send a message to $u_{i+1}$ (and also $u_{i+1}$ to receive this message) in round $r_{0}+i+1$. In turn, starting from round $r_{0}+i+2$, $u_{i+1}$ 's behavior may potentially be affected by this message.

We define the length of a spoil path $u_{0}, u_{1}, u_{2}, \ldots, u_{k}, v$ to be $k+1$. Intuitively, a spoil path is a potential path for $u_{0}$ to causally affect $v$, without going through $\alpha$ or $\beta$. Since Alice (Bob) will send to the other party all messages sent by $\alpha(\beta)$, paths going through $\alpha(\beta)$ are already taken care of. We intentionally define spoil paths in such a way that they can only be "blocked" by stable failures. This makes this definition consistent with the following intuition: If a node on a spoil path fails and if that failure is not a stable failure, then that node must be an epicenter itself and will already cause the spreading of spoiled nodes. Thus intuitively, such a non-stable failure can never block the spreading of spoiled nodes. The spoil distance of a node $v$ from an epicenter $u_{0}$ occurring at round $r_{0}$ is simply the length of the shortest spoil path from $u_{0}$ to $v$, or infinite if there is no such spoil path. For any epicenter $u_{0}$, we also define the spoil distance of $u_{0}$ from itself to be 0 . For any given round $r$, a node $v$ is spoiled in round $r$ with respect to Alice's input $X$ if $v$ is within spoil distance of $r-r_{0}$ hops from some epicenter occurring at round $r_{0}$ where $r_{0} \leq r$. By such definition, an epicenter with occurrence time of $r$ becomes first spoiled in round $r$, which is consistent with the intuition. We use $S_{A, X}(r)$ to denote the set of all spoiled nodes at round $r$ with respect to Alice's input $X$. We will prove in the next section that in each round $r$, Alice with input $X$ can simulate all unspoiled nodes (i.e., all nodes in $\bar{S}_{A, X}(r)$ ).

We similarly define the notion of epicenters, spoil paths, spoiled nodes, and $S_{B, Y}(r)$ for Bob.

The simulatability lemma. Given the above formal framework, we can now prove the following simple but useful lemma which we will repeatedly invoke later.

Lemma 12 Let $X$ be Alice's input and $Y$ be Bob's. Let $R$ be any positive integer where $\alpha \in \bar{S}_{A, X}(R)$ and $\beta \in \bar{S}_{B, Y}(R)$. Assume that Alice (Bob) always forwards to the other party the message sent by $\alpha(\beta)$ in a round whenever Alice (Bob) is able to simulate the execution of the SUM oracle protocol on $\alpha(\beta)$ for that round. Then for all $0 \leq r \leq R$, Alice can properly simulate the execution of the SUM oracle protocol on all nodes in $\bar{S}_{A, X}(r)$ for round $r$ and Bob can properly simulate all nodes in $\bar{S}_{B, Y}(r)$ for round $r$.

Proof: We do an induction on $r$. First, $\alpha \in \bar{S}_{A, X}(R)$ and $\beta \in \bar{S}_{B, Y}(R)$ imply $\alpha \in \bar{S}_{A, X}(r)$ and $\beta \in \bar{S}_{B, Y}(r)$ for all $0 \leq r \leq R . \bar{S}_{A, X}(0)$ simply contains all nodes, since there are no epicenters occurring in round 0 . Clearly, Alice can simulate all nodes for round 0 since the SUM protocol does nothing in round 0 and no failures happen in round 0. Similarly, for round 0 Bob can simulate all nodes in $\bar{S}_{B, Y}(0)$.

Assume that the claim holds for round $r$, and consider any node $v \in \bar{S}_{A, X}(r+1)$. We distinguish two cases:

- $v$ is not an epicenter for Alice's input $X$. Then Alice can uniquely determine both the (initial) value and the failure time of $v$. If the failure time is round $r+1$ or earlier, then Alice trivially simulates $v$ in round $r+1$ by doing nothing and we are done. Otherwise Alice knows that $v$ is alive in round $r+1$. 
- $v$ is an epicenter for Alice's input $X$. We claim that it is impossible for the occurrence time of this epicenter to be round $r+1$ or earlier, since otherwise $v$ would have been spoiled in round $r+1$ and thus would not be in $\bar{S}_{A, X}(r+1)$. Given that the occurrence time is round $r+2$ or later, it means that the occurrence time is not round 1. Thus $v$ is not a value epicenter and Alice must know $v$ 's initial value. Furthermore, while Alice cannot determine $v$ 's exact failure time, Alice knows for sure that the failure time of $v$ is round $r+2$ or later, and that $v$ is alive in round $r+1$.

Now we only need to prove that Alice can simulate $v$ in round $r+1$, given that Alice knows $v$ 's initial value and that $v$ is alive in round $r+1$.

We trivially have $v \in \bar{S}_{A, X}(r)$ and by inductive hypothesis, Alice can simulate $v$ from round 1 to $r$ (inclusive). It thus suffices to prove that Alice can generate the potential message that $v$ receives in round $r$ from some neighbor $u$, so that Alice can simulate $v$ in round $r+1$. We distinguish three cases for $u$. If $u$ is $\beta$ and since $\beta \in \bar{S}_{B, Y}(r)$, by inductive hypothesis, Bob can properly simulate $\beta$ for round $r$. By condition of the lemma, Bob must have forwarded the message from $\beta$ to Alice. Similarly if $u$ is $\alpha$ and since $\alpha \in \bar{S}_{A, X}(r)$, Alice can properly simulate $\alpha$ for round $r$ and generate the message herself. Finally, if $u \neq \alpha$ and $u \neq \beta$, we first show that $u$ must be in $\bar{S}_{A, X}(r)$, via a contradiction. Since $u$ sends a message in round $r$, $u$ must have not failed in round $r$ or earlier. In turn, $u$ must have not failed stably in round $r$ or earlier. Thus if $u \notin \bar{S}_{A, X}(r)$ (i.e., $u$ is spoiled in round $r$ ), then $v$ must be spoiled in round $r+1$, which contradicts with $v \in \bar{S}_{A, X}(r+1)$. Now given that $u \in \bar{S}_{A, X}(r)$, by inductive hypothesis Alice can simulate $u$ for round $r$ and generate $u$ 's message locally. Thus Alice has all the information needed to simulate $v$ for round $r+1$.

Similar arguments apply to Bob.

\section{C.3 Proof for Theorem 2 in Section 4}

Leveraging the previous formal framework, we can now prove Theorem 2 in Section 4.

Lemma 13 Consider the topology, valued nodes (with their values), and flaky nodes (with their failure times), as constructed in Appendix C.1. Under this construction and under $R=\frac{12}{c} \log n+\log n$, for all possible input $X$ of Alice's, we have $\alpha \in \bar{S}_{A, X}(R)$. Similarly, for all possible input $Y$ of Bob's, we have $\beta \in \bar{S}_{B, Y}(R)$.

Proof: Without loss of generality, we prove $\alpha \in \bar{S}_{A, X}(R)$. With respect to $X$, there are only two kinds of epicenters. The flaky node $\gamma_{i}^{\beta}(1 \leq i \leq n)$ is always a failure epicenter, occurring at round $t_{0}=\frac{6}{c} \log n+1$. One can easily verify that the spoil distance from $\gamma_{i}^{\beta}$ to $\alpha$ is at least $\frac{6}{c} \log n+\log n$. If $X_{i}=0$, then $\tau_{i}$ is a value epicenter with respect to $X$. The spoil distance from $\tau_{i}$ to $\alpha$ is at least $\frac{12}{c} \log n+\log n$, since the shortest possible spoil path (of length $\frac{6}{c} \log n+\log n$ ) is blocked by the stable failure of $\gamma_{i}^{\alpha}$.

Lemma 14 Consider any positive constant $c$, any $b \in[1,2-c]$, and any sufficiently large integer $N$. Let $n$ be the largest integer such that $n$ is a power of 2 and $\frac{9}{c} n \log n+3 n-2 \leq N$. There exists a connected topology $G$ with $N$ nodes, such that:

$$
\begin{aligned}
& \mathcal{R}_{0}^{\mathrm{syn}, \mathrm{ft}}(\mathrm{SUM}, G, b) \geq \frac{1}{2} \mathcal{R}_{0}^{\mathrm{Syn}}\left(\mathrm{UNIONSIZE}_{n}, b N\right) \\
& \mathcal{R}_{\epsilon, \frac{1}{3}}^{\mathrm{syn}, \mathrm{ft}}(\mathrm{SUM}, G, b) \geq \frac{1}{2} \mathcal{R}_{\epsilon, \frac{1}{3}}^{\mathrm{syn}}\left(\mathrm{UNIONSIZE}_{n}, b N\right)
\end{aligned}
$$

Proof: We first prove the lemma for $N=\frac{9}{c} n \log n+3 n-2$. We construct $G$ as in Figure 11, and let $R=\frac{12}{c} \log n+\log n$. Under the failure adversary constructed in Appendix C.1, let $\lambda=\max _{G^{\prime} \in \mathcal{G}} \Lambda\left(G^{\prime}\right)$ where $\mathcal{G}$ is the set of topologies that have ever appeared during the execution. We first prove that $R \geq(2-c) \lambda$ for all $n \geq 2$. Since in our construction all failures are injected at the same time, $\mathcal{G}$ actually only contains two topologies: one before failures and one after failures. It is easy to verify that for either $G^{\prime} \in \mathcal{G}, \Lambda\left(G^{\prime}\right)$ is at 
most $\frac{6}{c} \log n+2 \log n+1$. Here the $2 \log n$ term takes care of the need to avoid collision on the internal trees nodes - otherwise it would be only $\log n$ rounds. We now have:

$$
\frac{R}{\lambda} \geq \frac{\frac{12}{c} \log n+\log n}{\frac{6}{c} \log n+2 \log n+1}>2-c
$$

Next we reduce the UNIONSIZE $n$ problem (in the synchronous rounds setting) to SUM. Consider any (black-box) oracle protocol for Sum. Given input $X$ to Alice in $\mathrm{UnIONSIZE}_{n}$, Alice will simulate the execution of the oracle protocol on all nodes in $\bar{S}_{A, X}(r)$ at round $r$ for $0 \leq r \leq R$. Similarly, given input $Y$ to Bob, Bob simulates all nodes in $\bar{S}_{B, Y}(r)$. Furthermore, whenever $\alpha$ sends a message, Alice will forward that message to Bob. The same applies to Bob and $\beta$. Note that it is possible for Alice and Bob to send each other a message simultaneously in one round. By Lemma 13 and 12, such simulation is possible. When the oracle protocol terminates, which must be no later than round $(2-c) \lambda \leq R, \alpha$ and thus Alice will know the final result of the sum. By our construction, the zero-error result of the sum on $G$ exactly equals $\operatorname{UnIONSIZE}(X, Y)$, and thus any $(\epsilon, \delta)$-approximate result of the sum is also an $(\epsilon, \delta)$-approximate result of $\operatorname{UnIONSIZE}(X, Y)$. The total amount of communication between Alice and Bob is exactly the total number of bits sent by $\alpha$ and $\beta$ combined in the above simulation. Thus either $\alpha$ or $\beta$ must have sent at least half of the total number of bits sent by Alice and Bob. Together with the trivial property that $\lambda \leq N$, we now have:

$$
\begin{aligned}
& \mathcal{R}_{0}^{\mathrm{syn}, \mathrm{ft}}(\mathrm{SUm}, G, b) \geq \frac{1}{2} \mathcal{R}_{0}^{\mathrm{syn}}\left(\mathrm{UNIONSIZE}_{n}, b \lambda\right) \geq \frac{1}{2} \mathcal{R}_{0}^{\mathrm{syn}}\left(\mathrm{UNIONSIZE}_{n}, b N\right) \\
& \mathcal{R}_{\epsilon, \frac{1}{3}}^{\mathrm{syn}, \mathrm{ft}}(\mathrm{SUM}, G, b) \geq \frac{1}{2} \mathcal{R}_{\epsilon, \frac{1}{3}}^{\mathrm{syn}}\left(\mathrm{UNIONSIZE}_{n}, b \lambda\right) \geq \frac{1}{2} \mathcal{R}_{\epsilon, \frac{1}{3}}^{\mathrm{syn}}\left(\mathrm{UNIONSIZE}_{n}, b N\right)
\end{aligned}
$$

We still need to prove the lemma for $N>\frac{9}{c} n \log n+3 n-2$. Let $N_{1}=\frac{9}{c} n \log n+3 n-2$. We first construct a connected topology $G_{1}$ with $N_{1}$ nodes as in Figure 11. Next we add $N_{2}=N-N_{1}=O(n \log n)$ extra nodes to $G_{1}$ to obtain the topology $G$ with $N$ nodes. Those $N_{2}$ nodes will always have a value of 0 and will never fail. Since we want $G$ to be connected, we need to attach those $N_{2}$ nodes to some existing nodes in $G_{1}$. We want to do so carefully so that i) Lemma 13 continues to hold after adding those nodes, and ii) the length of an aggregation round is not affected by adding those nodes. These two properties will allow our earlier proof (for $N=\frac{9}{c} n \log n+3 n-2$ ) to carry over without modification. Specifically, we partition the $N_{2}$ nodes into $n$ equal-sized groups, with each group having $O(\log n)$ nodes. We then have each group form a binary tree of height $O(\log \log n)$. Finally, we attach the root of the $i$ th binary tree to the middle node (i.e., the degree-3 node) of the $i$ th $T$-structure in $G_{1}$.

It is trivial to verify that Lemma 13 continues to hold after adding those $N_{2}$ nodes in the above way. Next to understand why the length of an aggregation round is never affected by those extra $N_{2}$ nodes, consider a given $T$-structure and the binary tree attached to the middle node of that $T$-structure. Recall that the length of an aggregation round is the number of rounds needed for the deterministic tree-aggregation protocol in Section 3 to terminate. When running that protocol, the root of the binary tree here will send an aggregation message to the middle node of the $T$-structure in round $O(\log \log n)$. On the other hand, under any $G^{\prime}$ in $\mathcal{G}$ where $\mathcal{G}$ is the set of topologies that have ever appeared during the execution, that middle node will never receive any other aggregation message before round $\frac{3}{c} \log n-1$. Thus this extra binary tree i) is itself not a bottleneck for the tree-aggregation protocol to terminate, and ii) never potentially collides with other aggregation messages. In turn, this means that the length of an aggregation round in the execution under $G$ is the same as the length of an aggregation round in the execution under $G_{1}$. 
With the above two properties in $G$, we now know that our earlier proof for $N=\frac{9}{c} n \log n+3 n-2$ carries over to $N>\frac{9}{c} n \log n+3 n-2$ without modification.

Proof for Theorem 2: For any sufficiently large $N$, consider the $N$-node connected topology $G$ as constructed by Lemma 14. We trivially have:

$$
\begin{aligned}
& \mathcal{R}_{0}^{\mathrm{syn}, \mathrm{ft}}\left(\operatorname{Sum}_{N}, b\right) \geq \mathcal{R}_{0}^{\mathrm{syn}, \mathrm{ft}}(\operatorname{SUM}, G, b) \geq \frac{1}{2} \mathcal{R}_{0}^{\mathrm{syn}}\left(\mathrm{UNIONSIZE}_{n}, b N\right) \\
& \mathcal{R}_{\epsilon, \frac{1}{3}}^{\mathrm{syn}, \mathrm{ft}}\left(\operatorname{Sum}_{N}, b\right) \geq \mathcal{R}_{\epsilon, \frac{1}{3}}^{\mathrm{syn}, \mathrm{ft}}(\operatorname{SuM}, G, b) \geq \frac{1}{2} \mathcal{R}_{\epsilon, \frac{1}{3}}^{\mathrm{syn}}\left(\mathrm{UNIONSIZE}_{n}, b N\right)
\end{aligned}
$$

By Lemma 14, the $n$ in the above inequalities is the largest integer that is a power of 2 and satisfies $\frac{9}{c} n \log n+$ $3 n-2 \leq N$. Thus we have $N=\Theta(n \log n)$. Applying Corollary 10 gives:

$$
\begin{aligned}
& \mathcal{R}_{0}^{\text {syn,ft }}\left(\operatorname{Sum}_{N}, b\right) \geq \frac{1}{2} \mathcal{R}_{0}^{\text {syn }}\left(\operatorname{UNIONSIZE}_{n}, b N\right) \geq \frac{1}{2} \mathcal{R}_{0}^{\text {syn }}\left(\operatorname{UNIONSIZE}_{n},(2-c) N\right) \\
& =\Omega\left(\frac{n}{\log n}\right)=\Omega\left(\frac{N}{\log ^{2} N}\right) \\
& \mathcal{R}_{\epsilon, \frac{1}{3}}^{\mathrm{syn}, \mathrm{ft}}\left(\operatorname{Sum}_{N}, b\right) \geq \frac{1}{2} \mathcal{R}_{\epsilon, \frac{1}{3}}^{\mathrm{syn}}\left(\mathrm{UNIONSIZE}_{n}, b N\right) \geq \frac{1}{2} \mathcal{R}_{\epsilon, \frac{1}{3}}^{\mathrm{syn}}\left(\operatorname{UNIONSIZE}_{n},(2-c) N\right) \\
& =\Omega\left(\frac{1}{\epsilon^{2} \log n}\right)=\Omega\left(\frac{1}{\epsilon^{2} \log N}\right), \text { for } \epsilon=\Omega\left(\frac{\sqrt{\log N}}{\sqrt{N}}\right)
\end{aligned}
$$

\section{Proof for Theorem 3 in Section 5}

The proofs in this section are based on the formal framework described in Appendix C.2.

Lemma 15 Consider the topology, valued nodes (with their values), and flaky nodes (with their failure times), as constructed in Section 5. Under this construction and under $R=q n$, for all possible input $X$ of Alice's, we have $\alpha \in \bar{S}_{A, X}(R)$. Similarly, for all possible input $Y$ of Bob's, we have $\beta \in \bar{S}_{B, Y}(R)$.

Proof: Without loss of generality, we prove $\alpha \in \bar{S}_{A, X}(R)$. We exhaustively consider all the epicenters with respect to Alice's input $X$. First, if $X_{i}=0$ (implying $Y_{i}$ must be 0 or 1 ), then $\tau_{i}$, $\sigma_{i}^{\beta}$, and $\gamma_{i}^{\beta}$ are the only epicenters on the $i$ th chain. The spoil distance from all these epicenters to $\alpha$ is infinite, since $\gamma_{i}^{\alpha}$ fails stably at the beginning of round $t_{0}$ and thus blocks the only possible spoil path.

Next if $X_{i}=q-1$, then $Y_{i}$ must be $q-1$ or $q-2$. If $q-1$ is even, then $\sigma_{i}^{\beta}$ (potentially occurring at round $t_{q-2}$ ) and $\gamma_{i}^{\beta}$ (potentially occurring at round $t_{q-1}$ ) are the only epicenters on the $i$ th chain. Again, $\gamma_{i}^{\alpha}$ fails stably at the beginning of round $t_{q-1}$ and thus blocks the only possible spoil path from those two epicenters to $\alpha$. If $q-1$ is odd, then $\sigma_{i}^{\beta}$ (potentially occurring at round $t_{q-1}$ ) and $\gamma_{i}^{\beta}$ (potentially occurring at round $t_{q-2}$ ) are the only epicenters on the $i$ th chain. Since $\sigma_{i}^{\alpha}$ fails stably at the beginning of round $t_{q-1}$, the only possible spoil path from $\gamma_{i}^{\beta}$ to $\alpha$ is blocked. The epicenter of $\sigma_{i}^{\beta}$ has an occurrence time of $t_{q-1}=q n+1>R$. Thus it can never cause $\alpha$ to be spoiled in round $R$.

Finally if $X_{i}$ is even and $0<X_{i}<q-1$, then $Y_{i}$ must be odd and thus $\sigma_{i}^{\beta}$ is the only epicenter on the $i$ th chain, with an occurrence time of round $t_{X_{i}-1}$. (Recall that the occurrence time is the earliest possible failure time.) But since $X_{i}$ is even, $\gamma_{i}^{\alpha}$ fails stably at the beginning of round $t_{X_{i}}$. This failure blocks the only possible spoil path from $\sigma_{i}^{\beta}$ to $\alpha$. The case where $X_{i}$ is odd and $0<X_{i}<q-1$ is similar. 
Lemma 16 Consider any $b \geq 1$ and any sufficiently large integer $N$. Let $n$ be the largest integer such that $2 n^{2}+3 n+2 \leq N$ and let $q=5 b$. There exists a connected topology $G$ with $N$ nodes, such that:

$$
\begin{aligned}
& \mathcal{R}_{0}^{\mathrm{syn}, \mathrm{ft}}(\mathrm{SUM}, G, b) \geq \frac{1}{2} \mathcal{R}_{0}^{\mathrm{syn}}\left(\mathrm{UNIONSIZECP}_{n, q}, b N\right) \\
& \mathcal{R}_{\epsilon, \frac{1}{5}}^{\mathrm{syn}, \mathrm{ft}}(\mathrm{SUM}, G, b) \geq \frac{1}{2} \mathcal{R}_{\epsilon, \frac{1}{5}}^{\mathrm{syn}}\left(\operatorname{UNIONSIZECP}_{n, q}, b N\right)
\end{aligned}
$$

Proof: We first prove the lemma for $N=2 n^{2}+3 n+2$. We construct $G$ as in Section 5. Let $R=t_{q-1}-1=$ $q n=5 b n$. Under our constructed failure adversary, let $\lambda=\max _{G^{\prime} \in \mathcal{G}} \Lambda\left(G^{\prime}\right)$ where $\mathcal{G}$ is the set of topologies that have ever appeared during the execution. We first prove that $R \geq b \lambda$. It is easy to verify that even if we pessimistically assume that all messages to $\alpha$ and $\beta$ have to be sent sequentially one by one, we still have $\lambda \leq(2 n+3)+(n+1)+n=4 n+4$. Thus we have $R=5 b n \geq b \lambda$ for sufficiently large $n$.

Next we reduce the $\mathrm{UNIONSIZECP}_{n, q}$ problem (in the synchronous rounds setting) to SUM, which will prove the lemma for $N=2 n^{2}+3 n+2$. This part of the proof is exactly the same as in the proof of Lemma 14, after substituting Lemma 13 with Lemma 15. Thus we do not repeat it here.

Finally, we still need to prove the lemma for $N>2 n^{2}+3 n+2$. Let $N_{1}=2 n^{2}+3 n+2$. We first construct a connected topology $G_{1}$ with $N_{1}$ nodes as in Section 5. Next we add $N_{2}=N-N_{1}=O(n)$ extra nodes to $G_{1}$ to obtain the topology $G$ with $N$ nodes. Those $N_{2}$ nodes will always have a value of 0 and will never fail. Same as in the proof of Lemma 14 , We want to add those $N_{2}$ nodes carefully so that i) Lemma 15 continues to hold, and ii) the length of an aggregation round is not affected. To do so, we partition the $N_{2}$ nodes into $n$ groups of size $O(1)$. All nodes in the $i$ th group have a degree of 1 and directly attach to the $\frac{n}{2}$ th node (from left to right) on the $i$ th chain in $G_{1}$.

It is trivial to verify that Lemma 15 continues to hold after adding those $N_{2}$ nodes in the above way. For the length of an aggregation round, note that each group has only $O(1)$ nodes and thus all nodes in the group will finish sending their aggregation messages by round $O(1)$. On the other hand, the $\frac{n}{2}$ th node on a chain will not receive any other aggregation messages before round $\frac{n}{2}-1$, under any $G^{\prime}$ in $\mathcal{G}$ where $\mathcal{G}$ is the set of topologies that have ever appeared during the execution. Same as in the proof of Lemma 14, these properties ensure that our earlier proof for $N=2 n^{2}+3 n+2$ carries over to $N>2 n^{2}+3 n+2$ without modification.

Proof for Theorem 3: Since the theorem trivially holds for $b>N$, we only need to prove the theorem for $b \leq N$. For any sufficiently large $N$, consider the $N$-node connected topology $G$ as constructed by Lemma 16. We trivially have:

$$
\begin{aligned}
& \mathcal{R}_{0}^{\mathrm{syn}, \mathrm{ft}}\left(\operatorname{Sum}_{N}, b\right) \geq \mathcal{R}_{0}^{\mathrm{syn}, \mathrm{ft}}(\operatorname{SuM}, G, b) \geq \frac{1}{2} \mathcal{R}_{0}^{\mathrm{syn}}\left(\operatorname{UNiONSIZECP}_{n, q}, b N\right) \\
& \mathcal{R}_{\epsilon, \frac{1}{5}}^{\mathrm{syn}, \mathrm{ft}}\left(\operatorname{SuM}_{N}, b\right) \geq \mathcal{R}_{\epsilon, \frac{1}{5}}^{\mathrm{syn}, \mathrm{ft}}(\operatorname{SuM}, G, b) \geq \frac{1}{2} \mathcal{R}_{\epsilon, \frac{1}{5}}^{\mathrm{syn}}\left(\operatorname{UNiONSIZECP}_{n, q}, b N\right)
\end{aligned}
$$

By Lemma 16, in the above inequalities, $q=5 b$ and $n$ is the largest integer satisfying $2 n^{2}+3 n+2 \leq N$. Thus we have $N=\Theta(\sqrt{n})$. Applying Theorem 4 gives:

$$
\begin{aligned}
\mathcal{R}_{0}^{\text {syn,ft }}\left(\operatorname{Sum}_{N}, b\right) & \geq \frac{1}{2} \mathcal{R}_{0}^{\mathrm{syn}}\left(\mathrm{UNIONSIZECP}_{n, q}, b N\right) \\
& =\Omega\left(\frac{n}{q^{2} \log n}\right)=\Omega\left(\frac{\sqrt{N}}{b^{2} \log N}\right) \\
\mathcal{R}_{\epsilon, \frac{1}{5}}^{\mathrm{syn}, \mathrm{ft}}\left(\operatorname{SuM}_{N}, b\right) & \geq \frac{1}{2} \mathcal{R}_{\epsilon, \frac{1}{5}}^{\mathrm{syn}}\left(\mathrm{UNIONSIZECP}_{n, q}, b N\right) \\
& =\Omega\left(\frac{1}{\epsilon q^{2} \log n}\right)=\Omega\left(\frac{1}{\epsilon b^{2} \log N}\right), \text { for } \epsilon=\Omega\left(\frac{1}{\sqrt[4]{N}}\right)
\end{aligned}
$$




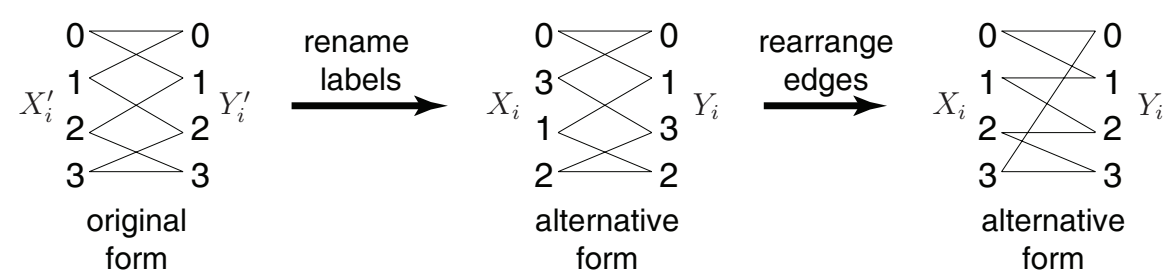

Figure 12: The alternative form of the cycle promise for $q=4$, used only in Appendix E.

\section{E Communication Complexity of UnIONSIZECP}

This section proves upper/lower bounds on the communication complexity of UNIONSIZECP. For discussion in this section, it will be convenient to consider an alternative form of the cycle promise (Figure 12). Consider any two length- $n$ strings $X$ and $Y$ where the characters in the strings are integers in $[0, q-1] . X$ and $Y$ satisfy the alternative form of the cycle promise iff for all $i$ 's where $1 \leq i \leq n$, either $Y_{i}=X_{i}$ or $Y_{i}=\left(X_{i}+1\right) \bmod q$.

Given $X^{\prime}$ and $Y^{\prime}$ satisfying the original cycle promise, Alice and Bob can always locally generate $X$ and $Y$, such that $X$ and $Y$ satisfy the alternative form of the cycle promise and $\operatorname{UniOnSIZECP}(X, Y)=$ UnionSiZECP $\left(X^{\prime}, Y^{\prime}\right)$. Specially to do so, Alice sets $X_{i}=X_{i}^{\prime} / 2$ for even $X_{i}^{\prime}$ and $X_{i}=q-\left(X_{i}^{\prime}+1\right) / 2$ for odd $X_{i}^{\prime}$. Bob sets $Y_{i}=\left(q-Y_{i}^{\prime} / 2\right) \bmod q$ for even $Y_{i}^{\prime}$ and $Y_{i}=\left(Y_{i}^{\prime}+1\right) / 2$ for odd $Y_{i}^{\prime}$. Clearly, we have $X_{i}=0$ iff $X_{i}^{\prime}=0$, and $Y_{i}=0$ iff $Y_{i}^{\prime}=0$, which implies that $\operatorname{UnIONSIZECP}(X, Y)=\operatorname{UnIONSIZECP}\left(X^{\prime}, Y^{\prime}\right)$. It is easy to verify that $X$ and $Y$ satisfy the alternative form of the cycle promise. Finally, since the above mapping from $X^{\prime}\left(Y^{\prime}\right)$ to $X(Y)$ is a bijection, one can also construct a reverse mapping from $X(Y)$ to $X^{\prime}\left(Y^{\prime}\right)$. Given such mappings in both directions, we trivially know that the communication complexity of UNIONSIZECP with the original cycle promise is exactly the same as the communication complexity of UNIONSIZECP with the alternative form of the cycle promise.

Throughout this section, we will always consider UNIONSIZECP under this alternative form of the cycle promise.

\section{E.1 An $O\left(\frac{n}{q}\right)$ Upper Bound Protocol}

We first present our $O\left(\frac{n}{q}\right)$ upper bound (when $q \leq n$ ) protocol for $\mathcal{R}_{0}^{\operatorname{syn}}\left(\operatorname{UNIONSIZECP}_{n, q}, \operatorname{poly}(n)\right)$. Given input $X$ to Alice, let $j(0 \leq j \leq q-1)$ be the integer with the smallest occurrence count in $X$. (If there are multiple such $j$ 's, simply pick an arbitrary one.) Alice first sends Bob the value of $j$ and the set $Z=\left\{i \mid X_{i}=j\right\}$. This takes at most $O\left(\log q+\frac{n}{q} \log n\right)$ bits in one round, or $O\left(\frac{\log q}{\log n}+\frac{n}{q}\right)$ bits in $\operatorname{poly}(n)$ rounds [19]. For $q \leq n$, this becomes $O\left(\frac{n}{q}\right)$. Bob will now know both $X_{i}$ and $Y_{i}$ for all $i \in Z$. In particular, if $j=0$ or $j=q-1$, then Bob can already determine $\left\{i \mid X_{i}=Y_{i}=0\right\}$, and can locally compute the final result. If $j \neq 0$ and $j \neq q-1$, then for any index $i^{\prime} \notin Z$, Bob knows that $X_{i^{\prime}} \neq j$. Thus if $Y_{i^{\prime}}=j+1$, then $X_{i^{\prime}}$ must be $j+1$ as well. This observation enables one to apply the following trick. Alice locally calculates $h_{A}=\mid\left\{i^{\prime} \mid i^{\prime} \notin Z\right.$ and $\left.j+1 \leq X_{i^{\prime}} \leq q-1\right\} \mid$ and sends $h_{A}$ to Bob, using $\log n$ bits in one round, or $O(1)$ bits in $\operatorname{poly}(n)$ rounds [19]. Bob calculates $h_{B}=\mid\left\{i^{\prime} \mid i^{\prime} \notin Z\right.$ and $\left(j+1 \leq Y_{i^{\prime}} \leq q-1\right.$ or $\left.\left.Y_{i^{\prime}}=0\right)\right\} \mid$. Given that $X_{i^{\prime}} \neq j$ for $i^{\prime} \notin Z$, one can easily verify from the cycle promise that $h_{B}-h_{A}$ is exactly the total number of indices $i$ where $X_{i}=Y_{i}=0$. The result for $\operatorname{UniONS\operatorname {SizCP}}(X, Y)$ is thus $n-\left(h_{B}-h_{A}\right)$. 


\section{E.2 Proof for Theorem 4 - Lower Bound via a Reduction from DisJointnessCP}

To lower bound the communication complexity of UNIONSIZECP, we will reduce from a new DiSJOINTNESSCP problem, which is the natural extension of the standard DISJOINTNESS problem on binary strings [24]. In DisjointnessCP ${ }_{n, q}(q \geq 2)$, again Alice and Bob each have a length- $n$ string $X$ and $Y$ as input, where the characters in the strings are integers in $[0, q-1]$. $X$ and $Y$ here satisfy the alternative form of the cycle promise as in UNIONSIZECP. Alice and Bob aim to determine whether there exists any $i$ where $X_{i}=0$ and $Y_{i}=0$, and they output 0 iff there exists such $i$. For convenience later, different from UnIONSizECP, for DisjointNESSCP we require both Alice and Bob to know the final result. Recall from Appendix A that the notation $\mathcal{R}_{0, \delta}$ simply means $\mathcal{R}_{\epsilon, \delta}$ with $\epsilon=0$. The next section will prove the following theorem on the communication complexity of DISJOINTNESSCP, via an information theoretic approach [4]:

Theorem $17 \mathcal{R}_{0}\left(\right.$ DisjointnessCP $\left._{n, q}\right)=\Omega\left(\frac{n}{q^{2}}\right)-O(\log n)$ and $\mathcal{R}_{0, \frac{1}{5}}\left(\right.$ DisjoInTnESsCP $\left._{n, q}\right)=\Omega\left(\frac{n}{q^{2}}\right)-$ $O(\log n)$.

Using the above theorem, one can obtain a lower bound on the communication complexity of UNIONSIZECP, under the setting without synchronized rounds, via a direct reduction:

Theorem $18 \mathcal{R}_{0}\left(\mathrm{UnIONSIZECP}_{n, q}\right)=\Omega\left(\frac{n}{q^{2}}\right)-O(\log n)$ and $\mathcal{R}_{\epsilon, \frac{1}{5}}\left(\mathrm{UnIONSIZECP}_{n, q}\right)=\Omega\left(\frac{1}{\epsilon q^{2}}\right)-O\left(\log \frac{1}{\epsilon}\right)$ for $\epsilon=\Omega\left(\frac{1}{\sqrt{n}}\right)$.

Proof: We first prove the theorem for $\epsilon \geq \frac{1}{\sqrt{2 n}} \cdot \mathcal{R}_{0}\left(\mathrm{UNIONSIZECP}_{n, q}\right)=\Omega\left(\frac{n}{q^{2}}\right)-O(\log n)$ follows from a reduction from DisjointNESSCP ${ }_{n, q}$. Consider any given protocol for UNIONSizeCP Un $_{n, q}$. Given inputs $X$ and $Y$ to DisjoINTNESSCP ${ }_{n, q}$, Alice and Bob directly invoke the protocol for $\mathrm{UNIONSIZECP}_{n, q}$, with $X$ and $Y$ being the inputs. Alice outputs 1 iff $\mathrm{UnIONSIZECP}_{n, q}$ returns $n$. Alice further sends Bob a single bit to inform Bob of this result. We have:

$$
\mathcal{R}_{0}\left(\operatorname{UNIONSIZECP}_{n, q}\right) \geq \mathcal{R}_{0}\left(\text { DisJOINTNESSCP}_{n, q}\right)-1=\Omega\left(\frac{n}{q^{2}}\right)-O(\log n) .
$$

$\mathcal{R}_{\epsilon, \frac{1}{5}}\left(\mathrm{UNIONSIZECP}_{n, q}\right)=\Omega\left(\frac{1}{\epsilon q^{2}}\right)-O\left(\log \frac{1}{\epsilon}\right)$ follows from a reduction from DisJoINTNESSCP $\frac{1}{2 \epsilon}, q$ Consider any given protocol for UNIONSIZECP $\mathrm{U}_{n, q}$. Given a length- $\frac{1}{2 \epsilon}$ input $X$ for DisJOINTNESSCP $\frac{1}{2 \epsilon}, q$, Alice locally generates a length- $n$ input $X^{\prime}$ by first replicating each character in $X$ for $\frac{1}{\epsilon}$ times, and then appending 0 until the length of $X^{\prime}$ reaches $n$. This is always possible since $\frac{1}{2 \epsilon^{2}} \leq n$. Bob generates $Y^{\prime}$ in a similar way. We now have:

- If DisjointnessCP $\frac{1}{2 \epsilon}, q(X, Y)=1$, then $\operatorname{UniONSIZECP}_{n, q}\left(X^{\prime}, Y^{\prime}\right)=\frac{1}{2 \epsilon^{2}}$.

- If DisjointnessCP $\frac{1}{2 \epsilon}, q(X, Y)=0$, then $\operatorname{UniOnSIZECP}_{n, q}\left(X^{\prime}, Y^{\prime}\right) \leq \frac{1}{2 \epsilon^{2}}-\frac{1}{\epsilon}$.

One can easily verify that for all $\epsilon>0$, we have $(1+\epsilon)\left(\frac{1}{2 \epsilon^{2}}-\frac{1}{\epsilon}\right)<(1-\epsilon) \frac{1}{2 \epsilon^{2}}$. Alice can now pick any value between $(1+\epsilon)\left(\frac{1}{2 \epsilon^{2}}-\frac{1}{\epsilon}\right)$ and $(1-\epsilon) \frac{1}{2 \epsilon^{2}}$ as the threshold. Alice outputs 1 for DisJoINTNESSCP $\frac{1}{2 \epsilon}, q(X, Y)$ iff UniOnSizECP ${ }_{n, q}\left(X^{\prime}, Y^{\prime}\right)$ returns a value above that threshold. Finally, Alice sends Bob a single bit to inform Bob of the result. We thus have:

$$
\mathcal{R}_{\epsilon, \frac{1}{5}}\left(\operatorname{UnIONSIZECP}_{n, q}\right) \geq \mathcal{R}_{0, \frac{1}{5}}\left(\text { DisjointnessCP }_{\frac{1}{2 \epsilon}, q}\right)-1=\Omega\left(\frac{1}{\epsilon q^{2}}\right)-O\left(\log \frac{1}{\epsilon}\right) .
$$
have:

We still need to cover the case for $\epsilon=\Omega\left(\frac{1}{\sqrt{n}}\right)$ but $\epsilon<\frac{1}{\sqrt{2 n}}$. For such $\epsilon$ (which is necessarily $\Theta\left(\frac{1}{\sqrt{n}}\right)$ ), we

$$
\mathcal{R}_{\epsilon, \frac{1}{5}}\left(\operatorname{UNIONSIZECP}_{n, q}\right) \geq \mathcal{R}_{\frac{1}{\sqrt{2 n}}, \frac{1}{5}}\left(\operatorname{UNIONSIZECP}_{n, q}\right)=\Omega\left(\frac{\sqrt{n}}{q^{2}}\right)-O(\log n)=\Omega\left(\frac{1}{\epsilon q^{2}}\right)-O\left(\log \frac{1}{\epsilon}\right)
$$


Proof for Theorem 4: Directly combine Theorem 18 and Lemma 8.

\section{E.3 Proving Theorem 17 via an Information Theoretic Approach}

This section proves Theorem 17 for the DisjoInTNESSCP problem. For convenience in the proofs later, we define DISJOINTNESSCP more formally as following:

Definition 19 (DisjoInTnESSCP) In the DisjoInTNESSCP ${ }_{n, q}$ problem, Alice and Bob respectively hold $X$ and $Y$, which are two strings of length $n$ satisfying $(X, Y) \in \mathcal{L}_{q}^{n}$ where

$$
\mathcal{L}_{q}^{n}=\left\{(X, Y) \mid X \in \mathbb{Z}_{q}^{n} \text { and } Y \in \mathbb{Z}_{q}^{n} \text { and }(Y-X) \in\{0,1\}^{n}\right\} .
$$

The goal is to compute the function DisJOINTNESSCP ${ }_{n, q}: \mathcal{L}_{q}^{n} \rightarrow\{0,1\}$ defined as

$$
\operatorname{DiSJOINTNESSCP}_{n, q}(X, Y)= \begin{cases}0 & \exists i \in\{1,2, \ldots, n\} \text { such that } X_{i}=Y_{i}=0 \\ 1 & \text { otherwise }\end{cases}
$$

Our proof for Theorem 17 will be almost entirely based on the information theoretic approach from [4]. In this approach, the information complexity of a function is used to lower bound the communication complexity of that function. Under certain conditions, it is further shown that the conditional information complexity of a function is a lower bound on the function's information complexity. Next, under certain conditions, the approach establishes a direct-sum result between the conditional information complexity of a function (e.g., DISJOINTNESSCP $\mathrm{S}_{n, q}$ ) and the conditional information complexity of its constituent primitive function (e.g., DISJOINTNESSCP $1, q$ ). Finally, the approach also provides some tools for reasoning about the conditional information complexity of such constituent primitive functions. The final lower bound on communication complexity obtained via this approach is for private-coin randomized protocols only. Since we will need a lower bound for public-coin protocols, at the end of this section, we will apply the well-known result from Newman [27] to convert this lower bound to a public-coin setting. Recall from Appendix A that the notation $\mathcal{R}_{0, \delta}$ simply means $\mathcal{R}_{\epsilon, \delta}$ with $\epsilon=0$. We define $\mathcal{R}_{0, \delta}^{\text {pri }}$ (DisJoINTNESSCP ${ }_{n, q}$ ) to be the same as $\mathcal{R}_{0, \delta}\left(\right.$ DisjOINTNESSCP$\left._{n, q}\right)$, except that $\mathcal{R}_{0, \delta}^{\text {pri }}$ is for private-coin protocols.

In the next, we first summarize the definitions and lemmas that we will use in this information theoretic approach. All these definitions (Definition 20 to 24) and lemmas (Lemma 25 to 28) are directly adapted from [4], and are not our contribution. See [4] for a more detailed discussion.

Definition 20 (Decomposable functions) (Adapted from [4]) If there are functions $h: \mathcal{L}_{q}^{1} \rightarrow\{0,1\}$ and $g:\{0,1\}^{n} \rightarrow\{0,1\}$ such that DisjointNESSCP ${ }_{n, q}(X, Y)=g\left(h\left(X_{1}, Y_{1}\right), h\left(X_{2}, Y_{2}\right), \ldots, h\left(X_{n}, Y_{n}\right)\right)$, then we say that DISJOINTNESSCP $n, q$ is $g$-decomposable with primitive $h$. When the context is clear, we simply say that DisJoINTNESSCP ${ }_{n, q}$ is decomposable with primitive $h$.

We construct $g$ as $g\left(x_{1}, x_{2}, \ldots, x_{n}\right)=\prod_{i=1}^{n} x_{i}$. Then according to above definition, DisJoINTNESSCP ${ }_{n, q}$ is a decomposable function with primitive $h=$ DISJOINTNESSCP $_{1, q}$. For convenience, from now on in this section, $h$ stands for the function DisjointnessCP $1, q$. Namely $h(x, y)=0$ if $x=y=0$, otherwise $h(x, y)=1$.

Definition 21 (Mixture of product distributions) (Adapted from [4]) For random variables $X_{i}$, $Y_{i}$, and $T_{i}$ $(1 \leq i \leq n)$, their joint distribution $\left(X_{i}, Y_{i}, T_{i}\right)$ is called a mixture of product distribution if conditioned on $T_{i}, X_{i}$ and $Y_{i}$ are independent. 
Let $\mathcal{T}=\{0,1\} \times\{1,2, . ., q-1\}$, and let $T_{i}(1 \leq i \leq n)$ be a random variable drawn uniformly randomly from $\mathcal{T}$. Let $X_{i}$ and $Y_{i}(1 \leq i \leq n)$ be two random variables depending on $T_{i}$ where:

- If $T_{i}=(0, j)$, then $X_{i}=j$ and $Y_{i}$ is drawn uniformly randomly from $\{j, j+1\}$.

- If $T_{i}=(1, j)$, then $Y_{i}=j$ and $X_{i}$ is drawn uniformly randomly from $\{j, j-1\}$.

All additions and subtractions in the above are on $\mathbb{Z}_{q}$. Note that under this construction, it is impossible for $X_{i}=Y_{i}=0$, which is intentional. Define $\zeta$ as the joint distribution of the above $\left(X_{i}, Y_{i}, T_{i}\right)$. Clearly, conditioned on $T_{i}, X_{i}$ and $Y_{i}$ are independent. Hence $\zeta$ is a mixture of product distribution for all $i$ 's $(1 \leq$ $i \leq n)$.

Definition 22 (Collapsing distribution) (Adapted from [4]) A distribution on $\mathcal{L}_{q}^{n}$ is called a collapsing distribution for DISJOINTNESSCP ${ }_{n, q}$ with respect to $h$, if DISJOINTNESSCP $_{n, q}$ is $g$-decomposable with primitive $h$, and if for all $(X, Y)$ 's in the support of that distribution, all $j$ 's where $1 \leq j \leq n$, and all $(x, y) \in \mathcal{L}_{q}^{1}$, the following holds:

$$
g\left(h\left(X_{1}, Y_{1}\right), \ldots, h\left(X_{j-1}, Y_{j-1}\right), h(x, y), h\left(X_{j+1}, Y_{j+1}\right), \ldots, h\left(X_{n}, Y_{n}\right)\right)=h(x, y)
$$

Define $\eta=\zeta^{n}$, and let $(X, Y, T) \sim \eta$. Consider the marginal distribution $\eta_{X Y}$ of $(X, Y)$ in $\eta$. Since $\left(X_{i}, Y_{i}, T_{i}\right) \sim \zeta, X_{i}$ and $Y_{i}$ cannot simultaneously be 0 , which means $h\left(X_{i}, Y_{i}\right)=1$. Hence for all $(X, Y)$ 's in the support of $\eta_{X Y}$, we have $h\left(X_{i}, Y_{i}\right)=1$ for all $i$ 's. This implies that $g(\ldots, h(x, y), \ldots)=h(x, y)$, and thus $\eta_{X Y}$ is a collapsing distribution for DISJOINTNESSCP ${ }_{n, q}$.

Definition 23 (Conditional information cost) (Adapted from [4]) Let $\mathcal{P}$ be any two-party private-coin randomized protocol for DISJOINTNESSCP ${ }_{1, q}$. Let $\left(X_{i}, Y_{i}, T_{i}\right) \sim \zeta$, which is a mixture of product distributions on $\mathcal{L}_{q}^{1} \times \mathcal{T}$. Given $X_{i}$ and $Y_{i}$ as the input to $\mathcal{P}$, the transmitted messages in $\mathcal{P}$ can be viewed as a random variable $\mathcal{P}\left(X_{i}, Y_{i}\right)$. The conditional information cost of $\mathcal{P}$ with respect to $\zeta$ (denoted as $\mathrm{CIC}_{\zeta}(\mathcal{P})$ ) is the mutual information between $\left(X_{i}, Y_{i}\right)$ and $\mathcal{P}\left(X_{i}, Y_{i}\right)$ conditioned on $T_{i}$. Or formally:

$$
\mathrm{CIC}_{\zeta}(\mathcal{P})=\sum_{t \in \mathcal{T}} I\left(\left\{\left(X_{i}, Y_{i}\right) ; \mathcal{P}\left(X_{i}, Y_{i}\right)\right\} \mid T_{i}=t\right) \operatorname{Pr}\left[T_{i}=t\right] .
$$

Here I stands for the standard notion of conditional mutual information [4].

Definition 24 (Conditional information complexity) (Adapted from [4]) Let $\mathcal{P}$ be any two-party privatecoin randomized protocol for DISJOINTNESSCP ${ }_{1, q}$, such that for any input $(x, y), \mathcal{P}$ can generate the correct result with probability at least $1-\delta$. The $\delta$-error conditional information complexity of DISJOINTNESSCP ${ }_{1, q}$ with respect to $\zeta$, denoted as $\mathrm{CIC}_{\zeta, \delta}$ (DISJOINTNESSCP $\left.1, q\right)$, is defined as the minimum conditional information cost across all possible $\mathcal{P}$ 's satisfying the earlier property.

Lemma 25 (Adapted from [4]) Consider DisJOINTNESSCP ${ }_{n, q}$, and the distribution $\zeta$, $\eta$, and $\eta_{X Y}$ as defined earlier. We already know DISJOINTNESSCP ${ }_{n, q}$ is a decomposable function with primitive DISJOINTNESSCP ${ }_{1, q}$, $\zeta$ is a mixture of product distribution on $\mathcal{L}_{q}^{1} \times \mathcal{T}$, and $\eta_{X Y}$ is a collapsing distribution for DISJOINTNESSCP ${ }_{n, q}$ with respect to DISJOINTNESSCP $\mathrm{P}_{1, q}$. We must have:

$$
\mathcal{R}_{0, \delta}^{\text {pri }}\left(\text { DisjointnessCP }_{n, q}\right) \geq n \times \mathrm{CIC}_{\zeta, \delta}\left(\text { DisjointnessCP }_{1, q}\right) .
$$

Lemma 26 (Adapted from [4]) Let $Z$ be a random variable uniformly randomly distributed on $\left\{z_{1}, z_{2}\right\}$, and let $\Phi\left(z_{1}\right)$ and $\Phi\left(z_{2}\right)$ be two additional random variables. If $\Phi\left(z_{1}\right)$ and $\Phi\left(z_{2}\right)$ are both independent of $Z$, then we have:

$$
I(Z ; \Phi(Z)) \geq H^{2}\left(\Phi_{z_{1}}, \Phi_{z_{2}}\right) .
$$

Here $\Phi_{z_{i}}$ is the distribution of $\Phi\left(z_{i}\right)$, and $H$ is the Hellinger distance [4] between two distributions. 
Lemma 27 (Adapted from [4]) For any two-party private-coin randomized protocol $\mathcal{P}$, let random variable $\mathcal{P}(x, y)$ denote the transmitted message in $\mathcal{P}$ under input $x$ and $y$. Let $\mathcal{P}_{x, y}$ denote the distribution of $\mathcal{P}(x, y)$. For all $x, x^{\prime}, y$, and $y^{\prime}$, we have:

$$
2 H^{2}\left(\mathcal{P}_{x, y}, \mathcal{P}_{x^{\prime}, y^{\prime}}\right) \geq H^{2}\left(\mathcal{P}_{x, y}, \mathcal{P}_{x^{\prime}, y}\right)+H^{2}\left(\mathcal{P}_{x, y^{\prime}}, \mathcal{P}_{x^{\prime}, y^{\prime}}\right)
$$

Lemma 28 (Adapted from [4]) Let $\mathcal{P}$ be any private-coin randomized protocol for DISJOINTNESSCP ${ }_{1, q}$, such that for any input $(x, y), \mathcal{P}$ can generate the correct result with probability at least $1-\delta$. For any two input pairs $(x, y) \in \mathcal{L}_{q}^{1}$ and $\left(x^{\prime}, y^{\prime}\right) \in \mathcal{L}_{q}^{1}$ where $\operatorname{DisjoinTnESSCP}_{1, q}(x, y) \neq \operatorname{DisjoinTNESSCP}_{1, q}\left(x^{\prime}, y^{\prime}\right)$, we have:

$$
H^{2}\left(\mathcal{P}_{x, y}, \mathcal{P}_{x^{\prime}, y^{\prime}}\right) \geq 1-2 \sqrt{\delta} .
$$

Having introduced the definitions and lemmas needed for the information theoretic arguments, we are now ready to prove Theorem 17.

Theorem $29 \mathcal{R}_{0, \delta}^{\mathrm{pri}}\left(\right.$ DISJOINTNESSCP$\left._{n, q}\right)=\Omega\left(\frac{n}{q^{2}}\right)$ for any positive constant $\delta \leq 0.22$.

Proof: Let $\mathcal{P}$ denote the optimal protocol with the minimum conditional information cost, across all possible two-party private-coin randomized protocols for DISJOINTNESSCP ${ }_{1, q}$ where for any input $(x, y)$, the protocol can always generate the correct result with probability at least $1-\delta$.

By Lemma 25 and Definition 23 and 24, we have:

$$
\begin{aligned}
& \mathcal{R}_{0, \delta}^{\text {pri }}\left(\text { DisjointnessCP }_{n, q}\right) \\
\geq & n \times \operatorname{CIC}_{\zeta, \delta}\left(\text { DisjointnessCP }_{1, q}\right) \\
= & n \times \operatorname{CIC}_{\zeta}(\mathcal{P}) \\
= & \frac{n}{2(q-1)} \sum_{t \in \mathcal{T}} I\left(\left\{X_{1}, Y_{1} ; \mathcal{P}\left(X_{1}, Y_{1}\right)\right\} \mid T_{1}=t\right) \\
= & \frac{n}{2(q-1)} \sum_{j=1}^{q-1}\left(I\left(\left\{X_{1}, Y_{1} ; \mathcal{P}\left(X_{1}, Y_{1}\right)\right\} \mid T_{1}=(0, j)\right)+I\left(\left\{X_{1}, Y_{1} ; \mathcal{P}\left(X_{1}, Y_{1}\right)\right\} \mid T_{1}=(1, j)\right)\right)
\end{aligned}
$$

Conditioned on $T_{1}=(0, j),\left(X_{1}, Y_{1}\right)$ is uniformly distributed on $\{(j, j),(j, j+1)\}$. Let $z_{1}=(j, j)$, $z_{2}=(j, j+1)$, and $Z=\left(X_{1}, Y_{1}\right)$. Lemma 26 tells us:

$$
I\left(\left\{X_{1}, Y_{1} ; \mathcal{P}\left(X_{1}, Y_{1}\right)\right\} \mid T_{1}=(0, j)\right) \geq H^{2}\left(\mathcal{P}_{j, j}, \mathcal{P}_{j, j+1}\right)
$$

Similarly, we have:

$$
I\left(\left\{X_{1}, Y_{1} ; \mathcal{P}\left(X_{1}, Y_{1}\right)\right\} \mid T_{1}=(1, j)\right) \geq H^{2}\left(\mathcal{P}_{j, j}, \mathcal{P}_{j-1, j}\right)
$$

Apply Cauchy inequality and triangle inequality, and we have:

$$
\begin{aligned}
\mathcal{R}_{0, \delta}^{\text {pri }}\left(\text { DisjointnessCP }_{n, q}\right) & \geq \frac{n}{2(q-1)} \sum_{j=1}^{q-1}\left(H^{2}\left(\mathcal{P}_{j, j}, \mathcal{P}_{j, j+1}\right)+H^{2}\left(\mathcal{P}_{j, j}, \mathcal{P}_{j-1, j}\right)\right) \\
& \geq \frac{n}{4(q-1)^{2}}\left(\sum_{j=1}^{q-1}\left(H\left(\mathcal{P}_{j, j}, \mathcal{P}_{j, j+1}\right)+H\left(\mathcal{P}_{j, j}, \mathcal{P}_{j-1, j}\right)\right)\right)^{2} \\
& \geq \frac{n}{4(q-1)^{2}}\left(\sum_{j=1}^{q-1} H\left(\mathcal{P}_{j, j+1}, \mathcal{P}_{j-1, j}\right)\right)^{2} \\
& =\frac{n}{4(q-1)^{2}}\left(H\left(\mathcal{P}_{1,2}, \mathcal{P}_{0,1}\right)+H\left(\mathcal{P}_{2,3}, \mathcal{P}_{1,2}\right)+\ldots+H\left(\mathcal{P}_{q-1,0}, \mathcal{P}_{q-2, q-1}\right)\right)^{2} \\
& \geq \frac{n}{4(q-1)^{2}} H^{2}\left(\mathcal{P}_{q-1,0}, \mathcal{P}_{0,1}\right)
\end{aligned}
$$


Next apply Lemma 27, and we have:

$$
\begin{aligned}
\mathcal{R}_{0, \delta}^{\text {pri }}\left(\text { DisjointnessCP }_{n, q}\right) & \geq \frac{n}{8(q-1)^{2}} H^{2}\left(\mathcal{P}_{q-1,0}, \mathcal{P}_{0,0}\right)+\frac{n}{8(q-1)^{2}} H^{2}\left(\mathcal{P}_{q-1,1}, \mathcal{P}_{0,1}\right) \\
& \geq \frac{n}{8(q-1)^{2}} H^{2}\left(\mathcal{P}_{q-1,0}, \mathcal{P}_{0,0}\right)
\end{aligned}
$$

Finally, apply Lemma 28, and we have:

$$
\mathcal{R}_{0, \delta}^{\text {pri }}\left(\text { DisjOINTNESSCP }_{n, q}\right) \geq \frac{n}{8(q-1)^{2}}(1-2 \sqrt{\delta})=\Omega\left(\frac{n}{q^{2}}\right)
$$

Proof for Theorem 17: According to Newman [27], ${ }^{9}$ we have:

$$
\mathcal{R}_{0,0.22}^{\text {pri }}\left(\text { DisJoInTNESSCP }_{n, q}\right) \leq \mathcal{R}_{0,0.2}\left(\text { DisjOINTNESSCP }_{n, q}\right)+O(\log n+\log \log q)
$$

Apply Theorem 29 and we have:

$$
\mathcal{R}_{0,0.2}\left(\text { DisjointnessCP }_{n, q}\right)=\Omega\left(\frac{n}{q^{2}}\right)-O(\log n+\log \log q)
$$

This lower bound is only non-trivial when $q<\sqrt{\frac{n}{\log n}}$. Thus we can discard the $\log \log q$ term for clarity:

$$
\mathcal{R}_{0,0.2}\left(\text { DISJOINTNESSCP }_{n, q}\right)=\Omega\left(\frac{n}{q^{2}}\right)-O(\log n)
$$

Finally, apply Lemma 7 and we have $\mathcal{R}_{0}\left(\operatorname{DiSJOINTNESSCP}_{n, q}\right)=\Omega\left(\frac{n}{q^{2}}\right)-O(\log n)$ as well.

\section{F Omitted Details from Section 6}

This section proves the completeness theorem in Section 6. We will present the proof in a top-down fashion, and elaborate the technical lemmas after describing the proof of the theorem. Following is a concise roadmap:

- Appendix F.1 proves Theorem 5 (i.e., the completeness theorem) in Section 6, while invoking Lemma 30.

- Appendix F.2 proves Lemma 30, while invoking Lemma 32.

- Appendix F.3 proves Lemma 32.

\section{F.1 Proof for Theorem 5 in Section 6}

Preparing for the proof. Recall the following formal notations from Section 6. Let $\mathcal{X}$ be Alice's input domain, and $\mathcal{Y}$ be Bob's. Let $\mathcal{L} \subseteq \mathcal{X} \times \mathcal{Y}$ be the set of all valid input pairs, given the promise in $\Pi$. If $\Pi$ has no promise, then $\mathcal{L}=\mathcal{X} \times \mathcal{Y}$. Given an input pair $(X, Y) \in \mathcal{L}$, an oblivious reduction has a reference setting specifying the value of each node in $G$. For any node $\tau$ where $\tau \neq \alpha$ and $\tau \neq \beta$, we define $\tau$ 's (value) assignment graph to be the bipartite graph where $\mathcal{X} \cup \mathcal{Y}$ are vertices and an edge $(X, Y)$ exists iff

\footnotetext{
${ }^{9}$ Newman's original result was only stated for functions, while here we are dealing with the partial functions of DISJOINTNESSCP. Nevertheless, Newman's original proof actually holds without modification to partial functions.
} 
$(X, Y) \in \mathcal{L}$. In addition, each edge $(X, Y)$ has a binary label which is the value of $\tau$ in the reference setting for $(X, Y)$.

With these notations in mind, consider the assignment graph of any node $\tau$ where $\tau \neq \alpha$ and $\tau \neq \beta$. Let $b^{\prime}=\lfloor\sqrt{b / 3}\rfloor$, which implies $b^{\prime} \geq 2$ since $b \geq 12$. We partition the vertices of $\tau$ 's assignment graph into $2 b^{\prime}$ disjoint subsets of $\mathcal{X}(0), \mathcal{Y}(0), \ldots, \mathcal{X}\left(b^{\prime}-1\right)$, and $\mathcal{Y}\left(b^{\prime}-1\right)$ in the following way. For all integer $i \in\left[0, b^{\prime}-3\right]$, we recursively define:

$$
\begin{aligned}
\mathcal{X}(0) & =\{X \mid \tau(X, Y)=0 \text { for some }(X, Y) \in \mathcal{L}\} \\
\mathcal{Y}(0) & =\{Y \mid \tau(X, Y)=0 \text { for some }(X, Y) \in \mathcal{L}\} \\
\mathcal{X}(i+1) & =\left(\mathcal{X} \backslash \cup_{j=0}^{i} \mathcal{X}(j)\right) \cap\{X \mid \tau(X, Y)=1 \text { for some }(X, Y) \in \mathcal{L} \text { where } Y \in \mathcal{Y}(i)\} \\
\mathcal{Y}(i+1) & =\left(\mathcal{Y} \backslash \cup_{j=0}^{i} \mathcal{Y}(j)\right) \cap\{Y \mid \tau(X, Y)=1 \text { for some }(X, Y) \in \mathcal{L} \text { where } X \in \mathcal{X}(i)\} \\
\mathcal{X}\left(b^{\prime}-1\right) & =\mathcal{X} \backslash \cup_{j=0}^{b^{\prime}-2} \mathcal{X}(j) \\
\mathcal{Y}\left(b^{\prime}-1\right) & =\mathcal{Y} \backslash \cup_{j=0}^{b^{\prime}-2} \mathcal{Y}(j)
\end{aligned}
$$

Figure 9 in Section 6 illustrates these sets for a given $\tau$ for $b^{\prime}=4$. Intuitively, any vertex with some 0-labeled incidental edge belongs to $\mathcal{X}(0)$ or $\mathcal{Y}(0)$. Thus an edge $(X, Y)$ always has a label of 1 if $X \notin \mathcal{X}(0)$ or $Y \notin \mathcal{Y}(0)$. We say that there are edges between two sets $\mathcal{X}(i)$ and $\mathcal{Y}(j)$ iff there exists some edge $(X, Y)$ in the assignment graph for some $X \in \mathcal{X}(i)$ and some $Y \in \mathcal{Y}(j)$. The next section will prove the following key lemma regarding $\tau$ 's assignment graph:

Lemma 30 In any oblivious reduction from $\Pi$ to $\mathrm{SUM}$, consider any node $\tau$ in $G$ where $\tau \neq \alpha$ and $\tau \neq \beta$. In $\tau$ 's assignment graph, there must be no edges labeled 1 between $\mathcal{X}(0)$ and $\mathcal{Y}(0)$, and no edges between $\mathcal{X}(i)$ and $\mathcal{Y}(i)$ for all $1 \leq i \leq b^{\prime}-2{ }^{10}$

Note that it is still possible for edges to exist between $\mathcal{X}\left(b^{\prime}-1\right)$ and $\mathcal{Y}\left(b^{\prime}-1\right)$. Intuitively, this lemma holds because if there existed an edge $(X, Y)$ satisfying the property described in the lemma, then the reference setting for $(X, Y)$ would need to have so many failures in $G$ such that $\tau$ would be disconnected from the root. Those failures are needed to ensure that Alice (Bob) can invoke the oracle on $\alpha(\beta)$ throughout the execution (i.e., to ensure that $\alpha$ and $\beta$ remain unspoiled). On the other hand, $\tau$ must have a value of 1 in the reference setting for such $(X, Y)$. This contradicts with the requirement on the reference settings in an oblivious reduction, which disallows disconnecting nodes with a value of 1 . Next we can use this lemma to prove the completeness theorem:

Proof for Theorem 5: By the condition in the theorem, there exists some oblivious reduction $\mathcal{P}$ from $\Pi$ to Sum for some topology $G$. Let the $N-2$ nodes other than $\alpha$ and $\beta$ in $G$ be $\tau_{1}, \tau_{2}, \ldots, \tau_{N-2}$. Consider any input pair $(X, Y) \in \mathcal{L}$. Let $\tau_{i}(X, Y), \alpha(X, Y)$, and $\beta(X, Y)$ be the values of $\tau_{i}$, $\alpha$, and $\beta$ in $\mathcal{P}$ 's reference setting for $(X, Y)$, respectively. Since the zero-error SuM result under the reference setting must be the same as $\Pi(X, Y)$ and since the reference setting never fails or disconnects nodes with a value of 1 , we have:

$$
\Pi(X, Y)=\alpha(X, Y)+\beta(X, Y)+\sum_{i=1}^{N-2} \tau_{i}(X, Y)
$$

We intend to reduce $\Pi$ to UnIONSIZECP ${ }_{N, b^{\prime}}$. To do so, Alice converts her input $X$ for $\Pi$ to a corresponding input $X^{\prime}$ of length $N$ for UNIONSIZECP in the following way, using only local knowledge. Let $X_{i}^{\prime}$ be the $i$ th character in the string $X^{\prime}$. Alice sets the last character $X_{N}^{\prime}$ to be 0 . Alice next leverages $\mathcal{P}$ to obtain the value of $\alpha(X, Y)$, without communicating with Bob. To do so, Alice invokes $\mathcal{P}$ using $X$ and then stops once

\footnotetext{
${ }^{10}$ Note that the lemma trivially holds when $b^{\prime}=2$.
} 
$\mathcal{P}$ needs to invoke the SUM oracle protocol (which Alice does not have). Doing so clearly does not incur any communication. By definition of oblivious reductions, $\mathcal{P}$ at this point must have decided, based on $X$, the (initial) value of node $\alpha$. Furthermore, this value must be the same as $\alpha(X, Y)$. Alice now obtains $\alpha(X, Y)$ purely based on local knowledge. Alice sets $X_{N-1}^{\prime}$ to this value. Next Alice needs to determine $X_{i}^{\prime}$ for $1 \leq i \leq N-2$. By definition of oblivious reductions, $\mathcal{P}$ needs to specify the reference setting corresponding to each input pair $(X, Y) \in \mathcal{L}$. Using such information in $\mathcal{P}$, Alice can determine the assignment graph of $\tau_{i}$ for $1 \leq i \leq N-2$. In $\tau_{i}$ 's assignment graph, Alice's current input $X$ must belong to exactly one of the subsets of vertices. Let $\mathcal{X}(j)$ be the subset to which $X$ belongs. Alice then sets $X_{i}^{\prime}=j$.

Bob constructs input $Y^{\prime}$ of length $N$ for UNIONSIZECP similarly, using only his local knowledge of $Y$. Specifically, Bob sets $Y_{N-1}^{\prime}=0$ and $Y_{N}^{\prime}=\beta(X, Y)$. Same as earlier, Bob can obtain $\beta(X, Y)$ via $\mathcal{P}$, without communicating with Alice. Next for each $i$ where $1 \leq i \leq N-2$, Bob sets $Y_{i}^{\prime}$ to be $j$ where $\mathcal{Y}(j)$ is the subset to which $Y$ belongs to, in $\tau_{i}$ 's assignment graph.

We next show that $X^{\prime}$ and $Y^{\prime}$ are strings satisfying the cycle promise with $q=b^{\prime}$. First, for $i=N-1$ or $N$, obviously $X_{i}^{\prime}$ and $Y_{i}^{\prime}$ satisfy the cycle promise. Next consider any $i \in[1, N-2]$ and $\tau_{i}$ 's assignment graph. Clearly $X_{i}^{\prime}$ and $Y_{i}^{\prime}$ are integer in $\left[0, b^{\prime}-1\right]$ for all such $i$. By construction of the assignment graph, we know that there are

- no edges between $\mathcal{X}(0)$ and $\mathcal{Y}(j)$ for all $j \geq 2$,

- no edges between $\mathcal{X}\left(b^{\prime}-1\right)$ and $\mathcal{Y}(j)$ for all $j \leq b^{\prime}-3$, and

- no edges between $\mathcal{X}\left(j_{1}\right)$ and $\mathcal{Y}\left(j_{2}\right)$ for all $1 \leq j_{1} \leq b^{\prime}-2$ and $\left|j_{1}-j_{2}\right| \geq 2$.

Furthermore, Lemma 30 shows that there are no edges between $\mathcal{X}(j)$ and $\mathcal{Y}(j)$ for all $1 \leq j \leq b^{\prime}-2$. Since $(X, Y) \in \mathcal{L}$, there must exist an edge between $X$ and $Y$ in $\tau_{i}$ 's assignment graph. Thus $X_{i}^{\prime}$ and $Y_{i}^{\prime}$ must satisfy the cycle promise.

Finally, consider any given protocol for UnIONSIZECP $\mathrm{P}_{N, b^{\prime}}$. Alice and Bob invoke that protocol using $X^{\prime}$ and $Y^{\prime}$ as inputs, respectively. We claim that UnIONSIZECP $\left(X^{\prime}, Y^{\prime}\right)$ can be used directly as the result of $\Pi(X, Y)$, since:

$$
\begin{aligned}
& \operatorname{UniOnSIZECP}\left(X^{\prime}, Y^{\prime}\right) \\
= & \mid\left\{i \mid(1 \leq i \leq N) \text { and }\left(X_{i}^{\prime} \neq 0 \text { or } Y_{i}^{\prime} \neq 0\right\}\right) \mid \\
= & \alpha(X, Y)+\beta(X, Y)+\mid\left\{i \mid(1 \leq i \leq N-2) \text { and }\left(X \notin \mathcal{X}(0) \text { for } \tau_{i} \text { or } Y \notin \mathcal{Y}(0) \text { for } \tau_{i}\right)\right\} \mid \\
= & \alpha(X, Y)+\beta(X, Y)+\mid\left\{i \mid 1 \leq i \leq N-2 \text { and } \tau_{i}(X, Y)=1\right\} \mid
\end{aligned}
$$

(by construction of the assignment graph and Lemma 30)

$$
=\alpha(X, Y)+\beta(X, Y)+\sum_{i=1}^{N-2} \tau_{i}(X, Y)=\Pi(X, Y)
$$

In the above derivation, we have leveraged Lemma 30 which shows that there are no edges labeled 1 between $\mathcal{X}(0)$ and $\mathcal{Y}(0)$. Together with the construction of the assignment graph, this means that $\tau_{i}(X, Y)=1$ if and only if in $\tau_{i}$ 's assignment graph, $X \notin \mathcal{X}(0)$ or $Y \notin \mathcal{Y}(0)$.

\section{F.2 Proof for Lemma 30}

Preparing for the proof. Consider any input pair $(X, Y) \in \mathcal{L}$ and the corresponding reference setting in the oblivious reduction from $\Pi$ to Sum. Define $\Phi(X, Y)$ to be the execution of the Sum oracle protocol under the reference setting and the (public) random string chosen by Alice and Bob in the oblivious reduction. For a given node $\tau$ in $G$ where $\tau \neq \alpha$ and $\tau \neq \beta$, we say that $\tau$ is disconnected form the root in an execution $\Phi(X, Y)$, if either $\tau$ fails during $\Phi(X, Y)$, or the root and $\tau$ are no longer in the same connected component at the end of $\Phi(X, Y)$. We have the following trivial lemma: 
Lemma 31 For any $(X, Y) \in \mathcal{L}$, if $\tau$ has a value of 1 under the reference setting for $(X, Y)$, then in $\Phi(X, Y)$, $\tau$ is never disconnected from the root.

Proof: Trivially follows from the requirement on the reference settings in oblivious reductions.

Proof for Lemma 30: The lemma trivially holds for $b^{\prime}=2$, and thus we only need to consider $b^{\prime} \geq 3$ (or equivalently $b \geq 27$ ). We prove this lemma via a contradiction and assume that the lemma does not hold. Intuitively, we will construct a path in $\tau$ 's assignment graph (not a path in $G$ ) where vertices on the path are individual inputs. Since it is a path in the assignment graph, by the definition of $\tau$ 's assignment graph, any two adjacent inputs on that path must form a valid input pair in $\mathcal{L}$. The path will start from some vertex in $\mathcal{X}(0)$ and end with some vertex in $\mathcal{Y}(0)$. Furthermore, all edges on the path have a label of 1 , and the path has no more than $2\left(b^{\prime}-1\right)-1$ hops. These properties can be later used to find a contradiction.

We now present the formal proof. If the lemma does not hold, then in the assignment graph of $\tau$, either there is an edge labeled 1 between $\mathcal{X}(0)$ and $\mathcal{Y}(0)$, or there is an edge (which must have a label of 1) between $\mathcal{X}(j)$ and $\mathcal{Y}(j)$ for some $j \in\left[1, b^{\prime}-2\right]$. We claim that in either case, we can find in the assignment graph a path $X^{(0)}, Y^{(1)}, X^{(1)}, Y^{(2)}, \ldots, X^{(k)}, Y^{(k+1)}$ for some $k \in\left[0, b^{\prime}-2\right]$, such that:

- $X^{(i)} \in \mathcal{X}$ for $0 \leq i \leq k$, and $Y^{(i)} \in \mathcal{Y}$ for $1 \leq i \leq k+1$,

- $X^{(0)} \in \mathcal{X}(0)$ and $Y^{(k+1)} \in \mathcal{Y}(0)$, and

- all edges in the path have a label of 1 .

If there is an edge labeled 1 between $\mathcal{X}(0)$ and $\mathcal{Y}(0)$, we simply set $k=0$ and let $X^{(0)}$ and $Y^{(1)}$ be the two endpoints of that edge, respectively. Our claim then trivially holds. If there is an edge $(X, Y)$ where $X \in \mathcal{X}(j)$ and $Y \in \mathcal{Y}(j)$ for some $j \in\left[1, b^{\prime}-2\right]$, then we set $k=j$. First consider the case where $k$ is even. By the construction of the assignment graph, $X$ must be connected with some vertex in $\mathcal{Y}(j-1)$, and that vertex must be connected to some vertex in $\mathcal{X}(j-2)$, and so on. Since $k$ is even, there must be some path (with exactly $k$ hops) in the assignment graph from $X$ to some $X^{(0)} \in \mathcal{X}(0)$. Similarly, there must be some path (with exactly $k$ hops) in the assignment graph from $Y$ to some $Y^{(k+1)} \in \mathcal{Y}(0)$. These two paths, together with the edge between $X$ and $Y$, exactly form the path needed by our claim. The case for odd $k$ is similar.

Given the above path $X^{(0)}, Y^{(1)}, X^{(1)}, Y^{(2)}, \ldots, X^{(k)}, Y^{(k+1)}$ (satisfying all the above properties), we define $\mathcal{I}$ (where $\mathcal{I} \subseteq \mathcal{L}$ ) to be the set of input pairs $(X, Y)$ such that $(X, Y)$ is an edge in that path. We also call $\mathcal{I}$ as the problematic input set. By construction of $\mathcal{I}$, we know that $\tau$ has a value of 1 in the reference setting for any $(X, Y) \in \mathcal{I}$. Lemma 32 next proves that for all $(X, Y) \in \mathcal{I}, \tau$ is disconnected from the root by the end of the execution of $\Phi(X, Y)$. This leads to a contradiction with Lemma 31 .

Lemma 32 Suppose $b \geq 27$. Consider the problematic input set $\mathcal{I}$ as constructed in the proof of Lemma 30. For all $(X, Y) \in \mathcal{I}, \tau$ is disconnected from the root by the end of the execution of $\Phi(X, Y)$.

\section{F.3 Proving Lemma 32}

Lemma 32 is non-trivial to prove, and we will need a lot of preparation work in this section before actually proving that lemma.

\section{F.3.1 Node $\alpha$ and $\beta$ Must Remain Unspoiled}

We first want to prove that node $\alpha(\beta)$ must remain unspoiled for Alice (Bob) in an oblivious reduction. To do so, we inherit the formal framework developed in Appendix C.2. Since for each input pair $(X, Y) \in \mathcal{L}$, there is a corresponding reference setting in the oblivious reduction, the notions of values and failure time 
of nodes in Appendix C.2 are still well-defined here. Namely, all we need to do is to replace the notion of "simulated execution under $(X, Y)$ " in Appendix C.2 by the notion of "reference setting for $(X, Y)$ ". ${ }^{11}$ All concepts defined in Appendix C.2 now carry over directly without modification. For example, a node $v$ is a value epicenter for Alice's input $X$ if its value in the reference setting is not uniquely determined by $X$.

Lemma 12 in Appendix C. 3 proved that Alice can simulate all unspoiled nodes. In this section, we intend to prove the reverse - namely, if a node is spoiled for Alice in a round $r$, then in an oblivious reduction, Alice can never invoke the oracle protocol on that node for round $r$. Since in an oblivious reduction Alice (Bob) is required to invoke the oracle on node $\alpha(\beta)$ throughout the execution, this in turn implies that $\alpha$ $(\beta)$ must remain unspoiled for Alice (Bob). Our proof will hinge upon the property of oblivious reductions, which requires Alice (Bob) to decide, beforehand, exactly up to which rounds she (he) will invoke the oracle on each node.

Lemma 33 Consider any oblivious reduction from $\Pi$ to SUM. If a node $v$ in $G$ is spoiled for Alice's input $X$ (Bob's input $Y$ ) in round $r^{\prime} \geq 0$, then when Alice (Bob) has the input $X(Y)$, Alice (Bob) will not invoke the oracle on $v$ for round $r^{\prime}$.

Proof: We only need to prove the part for Alice. Let $r \in\left[1, r^{\prime}\right]$ be the very first round during which $v$ is spoiled. It suffices to prove that Alice will not invoke the oracle on $v$ for round $r$ - since the oracle protocol carries internal state from round to round, Alice can never invoke the oracle for round $r^{\prime}$ without invoking the oracle for earlier rounds.

If round $r$ is the first round during which $v$ is spoiled, there must exist some epicenter $u_{0}$ with an occurrence time of $r_{0}\left(r_{0} \leq r\right)$ such that there exists a spoil path from $u_{0}$ to $v$ with exactly $l=r-r_{0}$ hops. To show that Alice will not invoke the oracle on $v$ for round $r$, we use an induction on $l$.

If $l=0, v$ itself must be an epicenter occurring at round $r$. We consider two cases. If $v$ is a value epicenter, then the occurrence time is round 1 and $r=1$. In an oblivious reduction, Alice needs to decide purely based on $X$, the input value of each node for which she will invoke the oracle for at least one round. This means that Alice must never invoke the oracle on $v$ - otherwise she risks deviating from the corresponding execution under the reference setting. Next if $v$ is a failure epicenter, then round $r$ (i.e., the occurrence time of the epicenter) must be the earliest possible failure time. This means that there exists Bob's inputs $Y$ and $Y^{\prime}$, such that $v$ 's failure time is exactly round $r$ in the reference setting for $(X, Y)$ and is after round $r$ in the reference setting for $\left(X, Y^{\prime}\right)$. If Alice decides that she will invoke the oracle on $v$ for round $r$, then again she risks deviating from the execution under the reference setting since the reference setting could be $(X, Y)$.

For the inductive step, assume that the lemma holds for all values up to $l$ and we consider $l+1$. Again, there exists some epicenter $u_{0}$ with an occurrence time of $r_{0}\left(r_{0} \leq r\right)$ such that there exists a spoil path from $u_{0}$ to $v$ with exactly $l+1$ hops. Consider the node $u$ immediately before $v$ in this spoil path. Then the length of the spoil path from $u_{0}$ to $u$ is exactly $l$ hops, and $u$ is spoiled in round $r-1$, where $r-1 \geq 1$. By the inductive hypothesis, Alice (with an input $X$ ) does not invoke the oracle on $u$ for round $r-1$. Next we prove via a contradiction and assume that Alice still invokes the oracle on $v$ for round $r$. Note that in an oblivious reduction, the only way for Alice to obtain the potential message sent in round $r-1$ by the oracle protocol on $u(u \neq \beta)$ is for Alice to invoke the oracle on $u$ for round $r-1$ herself. Thus for Alice to still invoke the oracle on $v$ for round $r, u$ must have failed in round $r-1$ or earlier in all the reference settings for all possible input pairs $(X, Y)$ given the current $X$. We claim that it is impossible for $u$ to fail exactly in round $r-1$ in all these reference settings, since otherwise this failure is a stable failure for $X$, and there would be no spoil path from $u_{0}$ to $v$ via $u$. Thus there must exist some $Y$ such that $u$ fails before round $r-1$. This in turn, means that the occurrence time of the epicenter $u$ is round $r-2$ or earlier. Thus $v$ is spoiled by $u$ in round $r-1$ or earlier, which contradicts with the fact that $r$ is the first round that $v$ becomes spoiled.

\footnotetext{
${ }^{11}$ These two notions are actually exactly the same. In Appendix C.2 there was no need to introduce the more formal notion of reference settings, so there we used the notion of simulated execution.
} 
Corollary 34 Consider any oblivious reduction from $\Pi$ to $\operatorname{SUM}$. For any input pair $(X, Y) \in \mathcal{L}, \alpha(\beta)$ must remain unspoiled for Alice's input $X$ (Bob's input $Y$ ) throughout the execution of $\Phi(X, Y)$.

Proof: Trivially follows from Lemma 33 and the fact that in an oblivious reduction, Alice (Bob) is required to invoke the oracle on $\alpha(\beta)$ throughout the entire execution.

The above corollary is all we need for the proofs next — we no longer need Lemma 33.

\section{F.3.2 Reasoning about Paths - Some Technical Lemmas}

This section proves a series of technical lemmas, which we will later use to prove Lemma 32. Throughout this section, we use $\mathcal{I}$ to denote the problematic input set as constructed in the proof of Lemma 30. We need to also introduce a few new notations. For any input $X$ of Alice's, if node $v$ has a stable failure, we use the function $F_{A}(X, v)$ to denote $v$ 's failure time. Otherwise $F_{A}(X, v)$ is undefined. Similarly define the function $F_{B}(Y, v)$. Recall the definition of aggregation rounds from Section 2. We define $\lambda(X, Y)$ to be the number of rounds in an aggregation round in $\Phi(X, Y)$. In other words, $\lambda(X, Y)=\max _{G^{\prime} \in \mathcal{G}} \Lambda\left(G^{\prime}\right)$ where $\mathcal{G}$ is the set of topologies that have ever appeared in the execution $\Phi(X, Y)$. Since an oblivious reduction needs to work for any arbitrary and black-box SUM oracle protocol whose time complexity is up to $b$ aggregation rounds for some given $b$, the oblivious reduction protocol needs to work even under the worst case where the execution of $\Phi(X, Y)$ takes as long as $b \lambda(X, Y)$ rounds.

Recall that Lemma 32 intends to claim that $\tau$ will be disconnected from the root in the execution of $\Phi(X, Y)$ for any $(X, Y) \in \mathcal{I}$. The main complexity in the proof comes from the fact that $G$ can be arbitrary. To show that $\tau$ will be disconnected from the root, we need to show that there does not exist any path for $\tau$ to reach the root when the execution ends. A second challenge is that a failure in the reference setting may or may not actually occur in $\Phi(X, Y)$ - if the execution terminates before the failure time of a node $v$, then node $v$ does not actually fail in $\Phi(X, Y)$. This is further complicated by the fact that the total number of rounds in $\Phi(X, Y)$ (i.e., $b \lambda(X, Y))$ depends on the value of $\lambda(X, Y)$, which is itself affected by failures.

Formal concepts for reasoning about paths in $G$. We next introduce some notations to reason about paths in $G$. A path $p$ in $G$ is a sequence of nodes $\left(v_{1}, v_{2}, \ldots, v_{k}\right)$ such that $k \geq 2$ and for all $i \in[1, k-1], v_{i+1}$ is a neighbor of $v_{i}$ in $G$. For any node $v, v \in p$ simply means that $v$ appears in $p$. A path $p$ is a simple path if no node appears more than once in the path. All paths we discuss will be simple paths. The length of a path $p$ (denoted as $|p|$ ) is defined as the number of nodes in $p$ minus 1 . Consider any node $\tau$ in $G$, where $\tau \neq \alpha$ and $\tau \neq \beta$. With respect to $\tau$, an $\alpha$-path is a path from $\tau$ to $\alpha$ without passing $\beta$. Formally, it is a path $\left(v_{1}, v_{2}, \ldots, v_{k}\right)$ satisfying $v_{1}=\tau, v_{k}=\alpha$, and $v_{i} \neq \beta$ for all $i \in[2, k-1]$. We similarly define $\beta$-paths with respect to $\tau$, as paths from $\tau$ to $\beta$ without passing $\alpha$. We will only discuss $\alpha$-paths and $\beta$-paths with respect to $\tau$, and thus we will drop the phrase "with respect to $\tau$ ". As we will easily prove later, since $\alpha$ is the root, any path $p$ from $\tau$ to the root must contain an $\alpha$-path or a $\beta$-path as a part.

For any $\alpha$-path or $\beta$-path $p$, we say that $p$ is cut in a certain round if some node (potentially $\tau$ ) in $p$ fails in or before that round. Given the problematic input set $\mathcal{I}$, we say that an $\alpha$-path or $\beta$-path $p$ is dummy if for all $(X, Y) \in \mathcal{I}, p$ is cut by the end of the execution $\Phi(X, Y)$. Otherwise $p$ is non-dummy. A non-dummy path $p$ may still be cut in the execution of $\Phi(X, Y)$ for some $(X, Y) \in \mathcal{I}$. Intuitively, a dummy path $p$ can be easily dismissed in our proofs later since we will be focusing on the input pairs in $\mathcal{I}$ and a dummy path is always cut in the corresponding executions. So usually we will only need to focus on non-dummy paths. We use $\mathbb{P}^{\alpha}\left(\mathbb{P}^{\beta}\right)$ to denote the set of all non-dummy $\alpha$-paths ( $\beta$-paths). Note that the paths in $\mathbb{P}^{\alpha}$ and $\mathbb{P}^{\beta}$ are not necessarily edge-disjoint or vertex-disjoint. For any given non-dummy $\alpha$-path or $\beta$-path $p$, we use $\mathbb{P}_{<p}^{\alpha}$ to denote the set of all paths in $\mathbb{P}^{\alpha}$ whose lengths are smaller than the length of $p$. Similarly define $\mathbb{P}_{<p}^{\beta}$.

For any path $p$ and integer $t$, we use $p_{A}(X, t)$ to denote the existence of some node $v \in p$ satisfying $F_{A}(X, v) \leq t|p|$. Intuitively, this means that the path $p$ will be cut in round $t|p|$ or earlier if Alice's input is $X$ and if the execution continues up to round $t|p|$. We similarly define $p_{B}(Y, t)$. We will often drop the 
subscripts in $p_{A}(X, t)$ and $p_{B}(Y, t)$ since they are usually obvious. We say that $\mathbb{P}_{<p}^{\alpha}(X, t)$ holds if either $\mathbb{P}_{<p}^{\alpha}$ is empty or if $p_{1}(X, t)$ holds for all $p_{1} \in \mathbb{P}_{<p}^{\alpha}$. Similarly define $\mathbb{P}_{<p}^{\alpha}(Y, t), \mathbb{P}_{<p}^{\beta}(X, t)$, and $\mathbb{P}_{<p}^{\beta}(Y, t)$. Also similarly define $\mathbb{P}^{\alpha}(X, t), \mathbb{P}^{\alpha}(Y, t), \mathbb{P}^{\beta}(X, t)$, and $\mathbb{P}^{\beta}(Y, t)$. We say that a non-dummy $\alpha$-path or $\beta$-path $p$ is a focal path for an input pair $(X, Y) \in \mathcal{I}$ iff both of the following two properties hold:

- $\mathbb{P}_{<p}^{\alpha}(X, b)$, or $\mathbb{P}_{<p}^{\alpha}(Y, b)$, or both hold.

- $\mathbb{P}_{<p}^{\beta}(X, b)$, or $\mathbb{P}_{<p}^{\beta}(Y, b)$, or both hold.

As we will easily prove later, a focal path $p$ has the nice property that all $\alpha$-paths and $\beta$-paths shorter than $p$ will by cut by the end of $\Phi(X, Y)$. If $\tau$ remains connected to the root, then $\lambda(X, Y)$ will be at least as large as the length of $p$ (formally proved later). This is often a necessary precondition for us to reason about various properties on $p$.

Some technical lemmas. In the next, we will prove a series of technical lemmas (Lemma 35 through 41), which will be need for the proof of Lemma 32 later.

Lemma 35 Any path $p$ from $\tau(\tau \neq \alpha$ and $\tau \neq \beta)$ to the root must contain an $\alpha$-path or a $\beta$-path as a part.

Proof: Trivially follows from the fact that $\alpha$ is the root. In fact, it is possible to prove the following stronger claim: $p$ must either be an $\alpha$-path itself or contains a $\beta$-path as a part. We chose to still state the lemma in its current form since we want the lemma to be symmetric for $\alpha$ and $\beta$.

Lemma 36 Consider any focal path $p$ for any input pair $(X, Y) \in \mathcal{I}$. We have $|p| \leq \lambda(X, Y)$.

Proof: By the construction of $\mathcal{I}$, we know that for any input pair $(X, Y) \in \mathcal{I}, \tau$ has a value of 1 in the execution of $\Phi(X, Y)$. Lemma 31 tells us that $\tau$ will not be disconnected from the root in $\Phi(X, Y)$. Let $p_{1}$ denote the shortest path from $\tau$ to the root at the end of the execution $\Phi(X, Y)$. By definition of an aggregation round, we know that the number of round in an aggregation round is no smaller than the root's eccentricity in the graph, and thus we have $\left|p_{1}\right| \leq \lambda(X, Y)$. Next consider the set of all $\alpha$-paths and $\beta$-paths. We claim that any $\alpha$-path or $\beta$-path that is shorter than $p$ will no longer exist (i.e., been cut) by the end of the execution $\Phi(X, Y)$. If this claim does hold, then notice that by Lemma 35, $p_{1}$ must contain an $\alpha$-path or a $\beta$-path. This means that $|p| \leq\left|p_{1}\right| \leq \lambda(X, Y)$.

We prove the earlier claim via a contradiction, and assume that there exist some $\alpha$-paths and/or $\beta$-paths that are shorter than $p$ and they still exist at the end of the execution $\Phi(X, Y)$. Let $p_{2}$ be the shortest one of those paths (if there are multiple such $p_{2}$ 's, simply pick an arbitrary one). Note that $p_{2}$ must be a nondummy path. Again since Lemma 35 tells us that $p_{1}$ must contain an $\alpha$-path or a $\beta$-path, we must have $\left|p_{2}\right| \leq\left|p_{1}\right| \leq \lambda(X, Y)$. If $p_{2} \in \mathbb{P}^{\alpha}$, then $p_{2} \in \mathbb{P}_{<p}^{\alpha}$ since $\left|p_{2}\right|<|p|$. Since $p$ is a focal path, we know that either $\mathbb{P}_{<p}^{\alpha}(X, b)$ or $\mathbb{P}_{<p}^{\alpha}(Y, b)$ hold, implying that either $p_{2}(X, b)$ or $p_{2}(Y, b)$ hold. Since $b\left|p_{2}\right| \leq b \lambda(X, Y)$, there will be a failure on $p_{2}$ by the end of the execution of $\Phi(X, Y)$. Contradiction. The case for $p_{2} \in \mathbb{P}^{\beta}$ is similar.

Lemma 37 For any input pair $(X, Y) \in \mathcal{I}$, it is impossible for $\mathbb{P}^{\alpha}(X, b)$ and $\mathbb{P}^{\beta}(Y, b)$ to both hold.

Proof: By Lemma 31, we know that $\tau$ will not be disconnected from the root in the execution of $\Phi(X, Y)$. This means there exists some path $p_{1}$ from $\tau$ to the root at the end of the execution. Lemma 35 tells us that $p_{1}$ must contain an $\alpha$-path or a $\beta$-path. This means that at the end of the execution, there is at least one $\alpha$-path or $\beta$-path that has not been cut. Let $p$ be the shortest $\alpha$-path or $\beta$-path that has not been cut at the end of the execution (if there are multiple such $p$ 's, simply pick an arbitrary one). Clearly $p$ must be a non-dummy path.

First consider the case where $p$ is a non-dummy $\alpha$-path. By how we pick $p$, we know that $p$ is a focal path for $(X, Y)$. Lemma 36 tells us that $|p| \leq \lambda(X, Y)$. Now since $p$ has not been cut at the end of the execution 
in round $b \lambda(X, Y)$, we know that $p(X, b)$ must not hold. This means that $\mathbb{P}^{\alpha}(X, b)$ does not hold. ${ }^{12}$ If $p$ is a non-dummy $\beta$-path, then one can similarly show that $\mathbb{P}^{\beta}(Y, b)$ does not hold.

Lemma 38 Consider any focal path $p$ for any input pair $(X, Y) \in \mathcal{I}$. In the execution of $\Phi(X, Y)$, for all $t \leq b-1$ :

- If some node in $p$ is spoiled for Alice's input $X$ in round $t|p|$ and if $p(X, t+1)$ does not hold, then all nodes in $p$ are spoiled for Alice's input $X$ in round $(t+1)|p|$.

- If some node in $p$ is spoiled for Bob's input $Y$ in round $t|p|$ and if $p(Y, t+1)$ does not hold, then all nodes in $p$ are spoiled for Bob's input $Y$ in round $(t+1)|p|$.

Proof: First, Lemma 36 tells us that $|p| \leq \lambda(X, Y)$, and thus $(t+1)|p| \leq b \lambda(X, Y)$. The remainder of the proof follows directly from the definition of spoil paths. In particular, by definition of spoil paths, only stable failures can block spoil paths.

Lemma 39 Consider any focal path $p$ for any input pair $(X, Y) \in \mathcal{I}$. In the execution of $\Phi(X, Y)$, for all $t \leq b-1$ :

- If $p(Y, t)$ holds and if $p(X, t+1)$ does not hold, then all nodes in $p$ are spoiled for Alice's input $X$ in round $(t+1)|p|$.

- If $p(X, t)$ holds and if $p(Y, t+1)$ does not hold, then all nodes in $p$ are spoiled for Bob's input $Y$ in round $(t+1)|p|$.

Proof: First, Lemma 36 tells us that $|p| \leq \lambda(X, Y)$, and thus $(t+1)|p| \leq b \lambda(X, Y)$. Without loss of generality, we only prove the first part of the lemma. By definition of $p(Y, t)$, we know that there exists some node $v \in p$ such that $F_{B}(Y, v) \leq t|p|<(t+1)|p| \leq b \lambda(X, Y)$. Since $p(X, t+1)$ does not hold, the failure of $v$ in round $F_{B}(Y, v)$ must not be a stable failure for Alice's input $X$. But since $v$ does fail in the reference setting for $(X, Y)$ in round $F_{B}(Y, v)$, it means that $v$ is an epicenter for Alice's input $X$. (Note that $v$ may still be either a value epicenter or a failure epicenter.) The occurrence time of this epicenter is round $F_{B}(Y, v)$ or earlier. This means that $v$ must be spoiled in round $F_{B}(Y, v) \leq t|p|$. Apply Lemma 38 then finishes the proof.

Lemma 40 Consider any focal path $p$ for any input pair $(X, Y) \in \mathcal{I}$. For all $t \leq b-1$ :

- If $p \in \mathbb{P}^{\alpha}$ and if $\tau$ is spoiled for Alice's input $X$ in round $t|p|$, then $p(X, t+1)$ must hold.

- If $p \in \mathbb{P}^{\beta}$ and if $\tau$ is spoiled for Bob's input $Y$ in round $t|p|$, then $p(Y, t+1)$ must hold.

Proof: First, Lemma 36 tells us that $|p| \leq \lambda(X, Y)$, and thus $(t+1)|p| \leq b \lambda(X, Y)$. Without loss of generality, we only prove the first part, via a contradiction. By Lemma 38, if $p(X, t+1)$ does not hold, then in the execution of $\Phi(X, Y)$, all nodes in $p$ are spoiled for Alice's input $X$ in round $(t+1)|p|$. Since $(t+1)|p| \leq b \lambda(X, Y)$, this means that $\alpha$ (which is in $p$ ) is spoiled by the end of the execution, which contradicts with Corollary 34 .

The next lemma's proof is based on elementary induction and does not use any advanced techniques. However, its proof is the most complex one in this section because while we are doing an induction on the input pairs in the problematic input set $\mathcal{I}$, we need to simultaneously reason about the multiple paths in $G$ and these two issues are entangled together. Later we will only need to use the second claim in the following lemma - the first claim in the lemma is proved so that we can carry both claims in the induction, which is critical for the proof to work.

\footnotetext{
${ }^{12}$ One can also simultaneously show that $\mathbb{P}^{\alpha}(Y, b)$ does not hold, though we do not need that claim.
} 
Lemma 41 Suppose $b \geq 27$. Let $X^{(0)}, Y^{(1)}, X^{(1)}, Y^{(2)}, \ldots, X^{(k)}, Y^{(k+1)}$ (where $\left.k+1 \leq\lfloor\sqrt{b / 3}\rfloor\right)$ be those inputs in the proof of Lemma 30 that correspond to the problematic input set $\mathcal{I}$. For any path $p \in \mathbb{P}^{\alpha}$, any integer $i \in[1, k+1]$, and any integer $t_{i} \in\left[0, b-2 i^{2}-2 i\right]$, we have:

- If $\mathbb{P}_{<p}^{\alpha}\left(X^{(i-1)}, t_{i}+4 i\right)$ and $\mathbb{P}_{<p}^{\beta}\left(Y^{(i)}, t_{i}\right)$ holds, then $p\left(X^{(i-1)}, t_{i}+4 i\right)$ holds.

- In particular, if $\mathbb{P}_{<p}^{\alpha}=\mathbb{P}_{<p}^{\beta}=\emptyset$, then $p\left(X^{(i-1)}, t_{i}+4 i\right)$ holds.

Proof: First, note that $i \leq k+1 \leq\lfloor\sqrt{b / 3}\rfloor$ and $b \geq 27$ imply $b-2 i^{2}-2 i \geq 0$. This means that the range for $t_{i}$ is never empty. We only prove the first part of the lemma, since the second part is the special case of the first part. We prove the first part via an induction on $i$. For $i=1$, since $t_{1}<t_{1}+4 \leq b$, we have $\mathbb{P}_{<p}^{\alpha}\left(X^{(0)}, b\right)$ and $\mathbb{P}_{<p}^{\beta}\left(Y^{(1)}, b\right)$. This means that $p$ is a focal path for the input pair $\left(X^{(0)}, Y^{(1)}\right)$. In the execution of $\Phi\left(X^{(0)}, Y^{(1)}\right), \tau$ has a value of 1 and is spoiled for Alice's input $X^{(0)}$ in round $1 \leq|p|$. Apply Lemma 40 and we have $p\left(X^{(0)}, 2\right)$, which implies $p\left(X^{(0)}, t_{1}+4\right)$ for all $t_{1} \in[0, b-4]$.

Now consider any $i \geq 2$, while assuming that the lemma holds for $i-1$. We are given the condition $\mathbb{P}_{<p}^{\alpha}\left(X^{(i-1)}, t_{i}+4 i\right)$ and $\mathbb{P}_{<p}^{\beta}\left(Y^{(i)}, t_{i}\right)$. We will prove $p\left(X^{(i-1)}, t_{i}+4 i\right)$ via a contradiction and assume that it does not hold. The final contradiction will be obtained by sequentially proving the following claims:

- Claim 1: $\mathbb{P}_{<p}^{\beta}\left(X^{(i-1)}, t_{i}+1\right)$ holds, which will be proved via the execution of $\Phi\left(X^{(i-1)}, Y^{(i)}\right)$.

- Claim 2: $\mathbb{P}_{<p}^{\beta}\left(Y^{(i-1)}, t_{i}+2\right)$ holds, which will be proved via the execution of $\Phi\left(X^{(i-1)}, Y^{(i-1)}\right)$.

- Claim 3: $\mathbb{P}_{<p}^{\alpha}\left(X^{(i-2)}, t_{i}+4 i-2\right)$ holds, which will be proved via the inductive hypothesis and implicitly via the execution of $\Phi\left(X^{(i-2)}, Y^{(i-1)}\right)$.

- Claim 4: $p\left(X^{(i-2)}, t_{i}+4 i-2\right)$ holds, which will be proved via the inductive hypothesis and implicitly via the execution of $\Phi\left(X^{(i-2)}, Y^{(i-1)}\right)$.

- Claim 5: $p\left(Y^{(i-1)}, t_{i}+4 i-1\right)$ holds, which will be proved via the execution $\Phi\left(X^{(i-2)}, Y^{(i-1)}\right)$.

- Claim 6: $p\left(X^{(i-1)}, t_{i}+4 i\right)$ holds, which will be proved via the execution of $\Phi\left(X^{(i-1)}, Y^{(i-1)}\right)$.

Figure 13 illustrates these 6 claims in an example topology.

Proving Claim 1. We prove $\mathbb{P}_{<p}^{\beta}\left(X^{(i-1)}, t_{i}+1\right)$ via a contradiction and let $p_{1}$ be any path in $\mathbb{P}_{<p}^{\beta}$ where $p_{1}\left(X^{(i-1)}, t_{i}+1\right)$ does not hold. We first show that $p$ and $p_{1}$ are both focal paths for the input pair $\left(X^{(i-1)}, Y^{(i)}\right)$. For $p$, we have $\mathbb{P}_{<p}^{\alpha}\left(X^{(i-1)}, t_{i}+4 i\right)$ and $\mathbb{P}_{<p}^{\beta}\left(Y^{(i)}, t_{i}\right)$. Since $t_{i}<t_{i}+4 i<b$, we know that $p$ is a focal path for $\left(X^{(i-1)}, Y^{(i)}\right)$. For $p_{1}$, since $\mathbb{P}_{<p_{1}}^{\alpha} \subset \mathbb{P}_{<p}^{\alpha}$ and $\mathbb{P}_{<p_{1}}^{\beta} \subset \mathbb{P}_{<p}^{\beta}$, by similar argument we know that $p_{1}$ is a focal path for $\left(X^{(i-1)}, Y^{(i)}\right)$ as well.

Next by the original condition, we have $p_{1}\left(Y^{(i)}, t_{i}\right)$. Invoke Lemma 39 for $p_{1}$ and we know that all nodes on $p_{1}$ (including $\tau$ ) are spoiled for Alice's input $X^{(i-1)}$ in the execution of $\Phi\left(X^{(i-1)}, Y^{(i)}\right)$ in round $\left(t_{i}+1\right)\left|p_{1}\right|<\left(t_{i}+1\right)|p|$. We next invoke Lemma 40 for $p$ and we know that $p\left(X^{(i-1)}, t_{i}+2\right)$ must hold, which implies $p\left(X^{(i-1)}, t_{i}+4 i\right)$. Contradiction.

Proving Claim 2. Consider any path $p_{1} \in \mathbb{P}_{<p}^{\beta}$. We first show that $p_{1}$ is a focal path for the input pair $\left(X^{(i-1)}, Y^{(i-1)}\right)$. From the original condition of $\mathbb{P}_{<p}^{\alpha}\left(X^{(i-1)}, t_{i}+4 i\right)$ and since $\mathbb{P}_{<p_{1}}^{\alpha} \subset \mathbb{P}_{<p}^{\alpha}$, we have $\mathbb{P}_{<p_{1}}^{\alpha}\left(X^{(i-1)}, t_{i}+4 i\right)$ which implies $\mathbb{P}_{<p_{1}}^{\alpha}\left(X^{(i-1)}, b\right)$. Next Claim 1 tells us that $\mathbb{P}_{<p}^{\beta}\left(X^{(i-1)}, t_{i}+1\right)$. By a similar argument, we have $\mathbb{P}_{<p_{1}}^{\beta}\left(X^{(i-1)}, b\right)$. Thus $p_{1}$ is a focal path for $\left(X^{(i-1)}, Y^{(i-1)}\right)$.

From Claim 1, we also have $p_{1}\left(X^{(i-1)}, t_{i}+1\right)$. Now invoke Lemma 39 for $p_{1}$. We will then have $p_{1}\left(Y^{(i-1)}, t_{i}+2\right)$, since otherwise all nodes in $p_{1}$ (including $\beta$ ) are spoiled for Bob's input $Y^{(i-1)}$ in the execution of $\Phi\left(X^{(i-1)}, Y^{(i-1)}\right)$ in round $\left(t_{i}+2\right)\left|p_{1}\right|$. 


\section{Example topology}

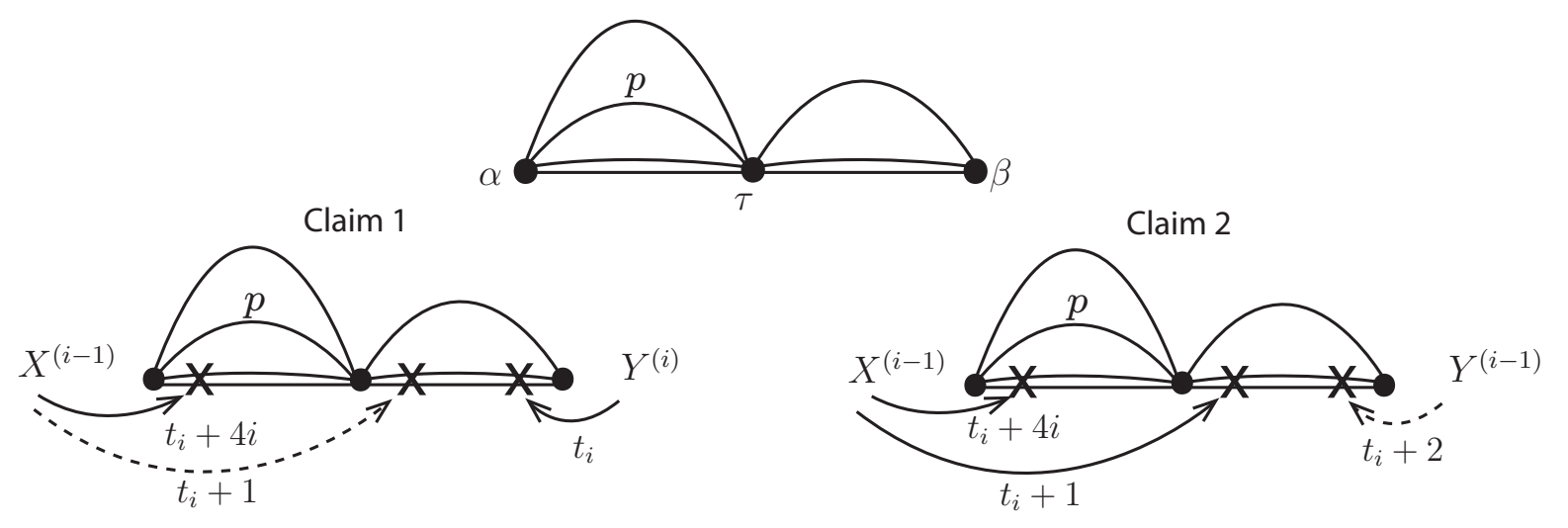

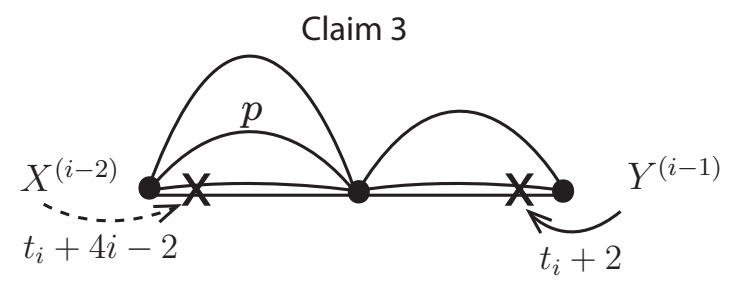

Claim 5

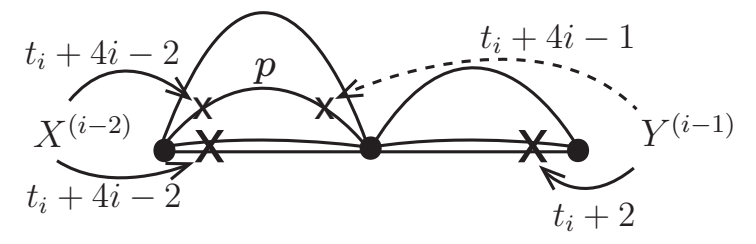

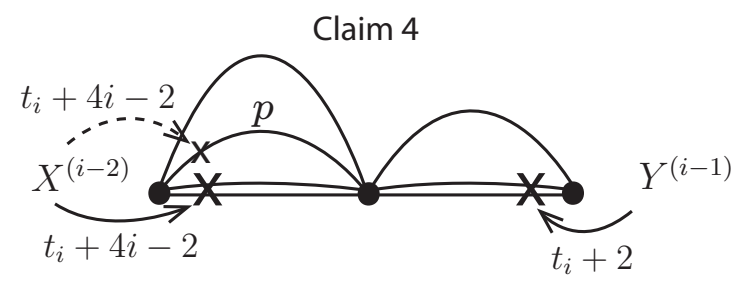

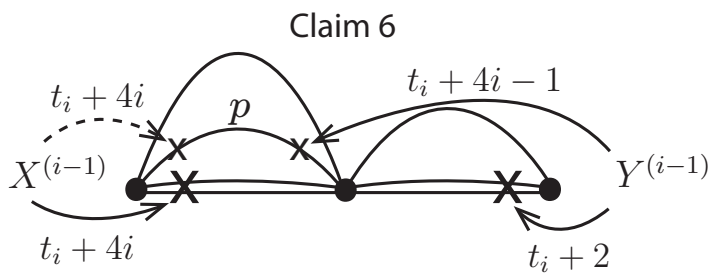

Figure 13: Illustration of the 6 claims proved in Lemma 41 in an example topology. For the given $\tau$, this example topology has $4 \alpha$-paths and $3 \beta$-paths. Nodes other than $\alpha, \beta$, and $\tau$ are not shown in the figure. The $\alpha$-path marked by $p$ is the path $p$ in the lemma. For clarity, we omit the labels for $\alpha, \beta$, and $\tau$ when illustrating the claims. For each of the claims, the figure indicates on the left the input (e.g., $\left.X^{(i-1)}\right)$ to Alice, and on the right the input to Bob. Solid arrows indicate those (stable) failures that we already know, given the corresponding input to Alice or Bob. Dashed arrows indicate those (stable) failures whose existence is proved in the corresponding claim.

Proving Claim 3. Prove by contradiction and assume that $\mathbb{P}_{<p}^{\alpha}\left(X^{(i-2)},\left(t_{i}+2\right)+4(i-1)\right)$ does not hold. Let $p_{1}$ be the shortest path (if there are multiple such $p_{1}$ 's, simply pick an arbitrary one) in $\mathbb{P}_{<p}^{\alpha}$ such that the $p_{1}\left(X^{(i-2)},\left(t_{i}+2\right)+4(i-1)\right)$ does not hold. We next want to invoke the inductive hypothesis for $i-1$ on $p_{1} \in \mathbb{P}^{\alpha}$ with $t_{i-1}=t_{i}+2$. Such invocation is possible since:

- $t_{i-1}=t_{i}+2 \leq b-2 i^{2}-2 i+2<b-2 i^{2}-2 i+4 i=b-2(i-1)^{2}-2(i-1)$.

- By definition of $p_{1}$, we have that $\mathbb{P}_{<p_{1}}^{\alpha}\left(X^{(i-2)},\left(t_{i}+2\right)+4(i-1)\right)$ holds.

- We know from Claim 2 that $\mathbb{P}_{<p}^{\beta}\left(Y^{(i-1)}, t_{i}+2\right)$ holds, implying that $\mathbb{P}_{<p_{1}}^{\beta}\left(Y^{(i-1)}, t_{i}+2\right)$ holds.

This invocation tells us that $p_{1}\left(X^{(i-2)},\left(t_{i}+2\right)+4(i-1)\right)$ holds, leading to a contradiction.

Proving Claim 4. We want to invoke the inductive hypothesis for $i-1$ on $p$ with $t_{i-1}=t_{i}+2$. Such invocation is possible since: 
- $t_{i-1}=t_{i}+2 \leq b-2 i^{2}-2 i+2<b-2 i^{2}-2 i+4 i=b-2(i-1)^{2}-2(i-1)$.

- Claim 3 gives us $\mathbb{P}_{<p}^{\alpha}\left(X^{(i-2)},\left(t_{i}+2\right)+4(i-1)\right)$.

- Claim 2 gives us $\mathbb{P}_{<p}^{\beta}\left(Y^{(i-1)}, t_{i}+2\right)$.

This invocation tells us that $p\left(X^{(i-2)},\left(t_{i}+2\right)+4(i-1)\right)$ holds.

Proving Claim 5. We first show that $p$ is a focal path for $\left(X^{(i-2)}, Y^{(i-1)}\right)$. We already have $\mathbb{P}_{<p}^{\alpha}\left(X^{(i-2)}, t_{i}+\right.$ $4 i-2)$ from Claim 3 and $\mathbb{P}_{<p}^{\beta}\left(Y^{(i-1)}, t_{i}+2\right)$ from Claim 2 . Since $t_{i}+2 \leq t_{i}+4 i-2 \leq b-2 i^{2}-2 i+4 i-2<$ $b$, we now know that $p$ is a focal path for $\left(X^{(i-2)}, Y^{(i-1)}\right)$. We next prove $p\left(Y^{(i-1)}, t_{i}+4 i-1\right)$ via a contradiction.

We already have $p\left(X^{(i-2)}, t_{i}+4 i-2\right)$ from Claim 4. Since $p\left(Y^{(i-1)}, t_{i}+4 i-1\right)$ does not hold, we can invoke Lemma 39 for $p$. That lemma tells us that in the execution of $\Phi\left(X^{(i-2)}, Y^{(i-1)}\right)$, all nodes in $p$ (including $\tau$ ) become spoiled for Bob's input $Y^{(i-1)}$ in round $\left(t_{i}+4 i-1\right)|p|$. This is a critical property which we will use later.

Next consider the two properties $\mathbb{P}^{\alpha}\left(X^{(i-2)}, t_{i}+8 i-4\right)$ and $\mathbb{P}^{\beta}\left(Y^{(i-1)}, t_{i}+4 i\right)$. By Lemma 37, it is impossible for both of them to hold, since otherwise they would imply that both $\mathbb{P}^{\alpha}\left(X^{(i-2)}, b\right)$ and $\mathbb{P}^{\beta}\left(Y^{(i-1)}, b\right)$ hold. Let $p_{1}$ be the shortest path (if there are multiple such $p_{1}$ 's, simply pick an arbitrary one) in $\mathbb{P}^{\alpha} \cup \mathbb{P}^{\beta}$ where $p_{1}\left(X^{(i-2)}, t_{i}+8 i-4\right)$ (if $\left.p_{1} \in \mathbb{P}^{\alpha}\right)$ or $p_{1}\left(Y^{(i-1)}, t_{i}+4 i\right.$ ) (if $p_{1} \in \mathbb{P}^{\beta}$ ) does not hold.

We consider two cases. If $p_{1} \in \mathbb{P}^{\beta}$, we will first show that $p_{1}$ is a focal path. By definition of $p_{1}$, we have $\mathbb{P}_{<p_{1}}^{\alpha}\left(X^{(i-2)}, t_{i}+8 i-4\right)$ and $\mathbb{P}_{<p_{1}}^{\beta}\left(Y^{(i-1)}, t_{i}+4 i\right)$ holds. Since $t_{i}+4 i \leq b-2 i^{2}-2 i+4 i<b, \mathbb{P}_{<p_{1}}^{\alpha}\left(X^{(i-2)}, b\right)$ and $\mathbb{P}_{<p_{1}}^{\beta}\left(Y^{(i-1)}, b\right)$ holds. This means that $p_{1}$ is a focal path for the input pair $\left(X^{(i-2)}, Y^{(i-1)}\right)$. We next want to show that $|p| \leq\left|p_{1}\right|$. By how we chose $p_{1}$, we know that $p_{1}\left(Y^{(i-1)}, t_{i}+4 i\right)$ does not hold. On the other hand, Claim 2 tells us that $\mathbb{P}_{<p}^{\beta}\left(Y^{(i-1)}, t_{i}+2\right)$ holds, implying that $\mathbb{P}_{<p}^{\beta}\left(Y^{(i-1)}, t_{i}+4 i\right)$ holds (since $i \geq 2$ ). Thus we must have $p_{1} \notin \mathbb{P}_{<p}^{\beta}$ and $\left|p_{1}\right| \geq|p|$. Finally, as shown earlier, in the execution of $\Phi\left(X^{(i-2)}, Y^{(i-1)}\right)$, the node $\tau$ must be spoiled for Bob's input $Y^{(i-1)}$ in round $\left(t_{i}+4 i-1\right)|p| \leq\left(t_{i}+4 i-1\right)\left|p_{1}\right|$. Now we can invoke Lemma 40, which shows that $p_{1}\left(Y^{(i-1)}, t_{i}+4 i\right)$ holds and thus leads to a contradiction.

For the second case where $p_{1} \in \mathbb{P}^{\alpha}$, we want to invoke the inductive hypothesis for $i-1$ on $p_{1}$ with $t_{i-1}=t_{i}+4 i$. Such invocation is possible since:

- $t_{i-1}=t_{i}+4 i \leq b-2 i^{2}-2 i+4 i=b-2(i-1)^{2}-2(i-1)$.

- By definition of $p_{1}$, we have that $\mathbb{P}_{<p_{1}}^{\alpha}\left(X^{(i-2)},\left(t_{i}+4 i\right)+4(i-1)\right)$ and $\mathbb{P}_{<p_{1}}^{\beta}\left(Y^{(i-1)}, t_{i}+4 i\right)$ holds.

The invocation gives us $p_{1}\left(X^{(i-2)},\left(t_{i}+4 i\right)+4(i-1)\right)$, leading to a contradiction.

Proving Claim 6. We first show that $p$ is a focal path for $\left(X^{(i-1)}, Y^{(i-1)}\right)$. From the original condition, we have $\mathbb{P}_{<p}^{\alpha}\left(X^{(i-1)}, t_{i}+4 i\right)$, which implies $\mathbb{P}_{<p}^{\alpha}\left(X^{(i-1)}, b\right)$. By Claim 2, we have $\mathbb{P}_{<p}^{\beta}\left(Y^{(i-1)}, t_{i}+2\right)$, which implies $\mathbb{P}_{<p}^{\beta}\left(Y^{(i-1)}, b\right)$. Thus $p$ is a focal path for $\left(X^{(i-1)}, Y^{(i-1)}\right)$.

Claim 5 gives us $p\left(Y^{(i-1)}, t_{i}+4 i-1\right)$. Now invoke Lemma 39 for $p$. That lemma tells us that $p\left(X^{(i-1)}, t_{i}+4 i\right)$ must hold, since otherwise all nodes in $p$ (including $\alpha$ ) will be spoiled for Alice's input $X^{(i-1)}$ in round $\left(t_{i}+4 i\right)|p|$.

\section{F.3.3 Proof for Lemma 32}

Using the technical lemmas proved in the previous section, we can now finally prove Lemma 32.

Proof for Lemma 32: By Lemma 35, a path from $\tau$ to the root must contain either an $\alpha$-path or a $\beta$-path. Thus to prove the lemma, it suffices to prove that all $\alpha$-paths and $\beta$-paths are dummy. Prove by contradiction and assume that some $\alpha$-paths and/or $\beta$-paths are non-dummy. Let $p$ be the shortest path among all such paths 
(if there are multiple such $p$ 's, simply pick an arbitrary one). This means that there exists some $(X, Y) \in \mathcal{I}$ such that $p$ is not cut by the end of the execution $\Phi(X, Y)$. Also by how we chose $p$, we trivially have $\mathbb{P}_{<p}^{\alpha}=\mathbb{P}_{<p}^{\beta}=\emptyset$.

Next first consider the case where $p$ is a non-dummy $\alpha$-path. For all $i$ where $1 \leq i \leq k+1 \leq\lfloor\sqrt{b / 3}\rfloor$, invoke the second claim in Lemma 41 with $t_{i}=0$ and we have $p\left(X^{(i-1)}, 4 i\right)$. Since $4 i \leq 4\lfloor\sqrt{b / 3}\rfloor<b$ when $b \geq 27$, this in turn implies $p\left(X^{(i-1)}, b\right)$ for $1 \leq i \leq k+1$. Next since $\mathbb{P}_{<p}^{\alpha}=\mathbb{P}_{<p}^{\beta}=\emptyset$, we trivially know that $p$ is a focal path for $(X, Y)$. Invoke Lemma 36 and we have $|p| \leq \lambda(X, Y)$. Together with $p(X, b)$, we know that $p$ will be cut by the end of the execution of $\Phi(X, Y)$. Contradiction.

For the second case where $p$ is a $\beta$-path, the proof is entirely symmetric. In particular, the only difference between $\alpha$ and $\beta$ is that $\alpha$ is the root while $\beta$ is not. However, we only used the fact that $\alpha$ is the root in the proof of Lemma 35. Lemma 35 itself is already symmetric for $\alpha$ and $\beta$. In other words, if we view the proof for Lemma 35 as a black-box, then $\alpha$ and $\beta$ are entirely symmetric throughout Appendix F.3.

\section{G Omitted Details from Section 7}

This section presents the omitted details from Section 7 for obtaining the FT lower bound of SUM under unrestricted $b$, while removing the strong gossip assumption. We will first precisely describe the lower bound topology and the adversary, while assuming that each node can take an integer value from some properly defined domain (instead of only binary values). Next we formally define the probing game, and then prove a strong connection from SUM protocols to strategies in the probing game. We then prove a lower bound on the probing game, which in turn leads to a lower bound on the FT communication complexity of SUM. Finally, we prove that this FT lower bound can be easily generalized to cases where each node can only have a binary value instead of an integer value.

\section{G.1 Topology and Adversary}

Here we assume that each node can take an integer value in the domain of $[0,3 n-1]-$ this assumption will be removed later. We construct our lower bound topology starting from a clique with $n+1$ nodes, with $n$ being a power of 2 . One of these nodes will be the root, while all other nodes are called worker nodes. We next insert a degree-two dummy node in the middle of each edge in the previous clique, so that failing the dummy node essentially fails the corresponding edge. ${ }^{13}$ The topology thus has total $\frac{(n+1)(n+2)}{2}$ nodes. Each worker node has an integer value in $[0,3 n-1]$. All other nodes have value of 0 . We use a vector $W=\left(w_{1}, w_{2}, \ldots, w_{n}\right)$ to denote the input (to the system), where $w_{i} \in[0,3 n-1]$ is the input value of worker node $i$.

Our adversary is deterministic but adaptive. The adversary conceptually partitions the worker nodes into groups, where each group has some group members and one group member is the group leader. Group membership and leadership may change whenever the adversary inject failures. Initially each worker is in its own group, with itself being the sole member (and thus the leader). The adversary keeps track of the set $L$ of current leaders. A leader in $L$ is marked once it sends a message. Once the number of marked leaders reaches $\frac{|L|}{2}$, the adversary pairs up each unmarked leader node $j$ with a distinct marked leader node $i$. In cases where multiple leaders send messages in the same round, the adversary will mark them sequentially (by their ids), until the number of marked leaders becomes exactly $\frac{|L|}{2}$. Next for each such node $j$, at the beginning of the next round, the adversary fails all dummy nodes connecting node $j$ to the root or connecting node $j$ to other leader nodes, except the dummy node connecting node $j$ to node $i$. (Note that the adversary does

\footnotetext{
${ }^{13}$ Under this construction, the total number of failures injected by our adversary later will reach $\Theta(N)$, where $N$ is the graph size. To obtain the same asymptotic FT lower bound while injecting only $o(N)$ failures, one only needs to instead insert log $n$ dummy nodes on each edge and fail only one of them.
} 
not fail any dummy nodes connecting node $j$ to non-leader nodes.) Next node $i$ 's group and node $j$ 's group are conceptually merged into one new group, with node $i$ being the new group leader. Finally, the adversary

updates $L$ to be the set of those $\frac{|L|}{2}$ leaders of the new groups, clears all the marks on those leaders, and repeats the above process until $|L|$ reaches 1 or the protocol terminates.

\section{G.2 A Strong Connection between SUM and The Probing Game}

We define the probing game as following. The probing game is played by a single player, against an input $W=\left(w_{1}, w_{2}, \ldots, w_{n}\right)$ where $w_{i}$ is an integer in $[0,3 n-1] . W$ is initially not known to the player, but the player knows $n$ and the domain $[0,3 n-1]$. The player proceeds in rounds. In each round, the player may choose to sequentially do zero, one, or multiple probes, where each probe is in the form of a tuple $(i, j)$. The outcome of the probe $(i, j)$ is a hit if $w_{i}=j$. Otherwise it is a miss. For each probe, the player may adaptively choose what probe to do (i.e., choose $i$ and $j$ ), based on the probing outcomes in previous rounds and also the probing outcomes so far in the current round. The goal of the player is to determine $\sum_{i=1}^{n} w_{i}$ based on the outcomes of the probes, while minimizing the total number of hits. Note that the player is not concerned with the total number of probes. For convenience later, we require that the player never does the same probe multiple times. In addition, if there has been a hit $(i, j)$, then the player does not further probe $\left(i, j^{\prime}\right)$ for any $j^{\prime}$ since the player has already learned $w_{i}$ precisely.

Theorem 42 below proves a strong connection from deterministic protocols for SUM to the probing game. This theorem reveals that under our adversary, while a SuM protocol on the surface has a lot of flexibility to apply various tricks, what the protocol fundamentally can do is no different from the system doing probes and having some leader node send a message if the probe is a hit. As a result, the number of hits in the probing game will be no larger than the total number of messages sent by the leaders.

Theorem 42 Assume that $n$ is a power of 2. Given any deterministic SUm protocol $\mathcal{P}$, there always exists an (adaptive) probing strategy $\mathcal{S}$ for the player in the probing game that satisfies the following property. For any input $W$, the player using $\mathcal{S}$ in the probing game against $W$ always generates the same result as the result generated by the SUM protocol $\mathcal{P}$ running against $W$ under the topology and adversary in Appendix G.1. Furthermore, if the total number of hits in the probing game when using $\mathcal{S}$ against $W$ reaches $n$, then the maximum number of bits sent by a node, across all nodes when running $\mathcal{P}$ against $W$ under the topology and adversary in Appendix G.1, is at least $\log n+1$.

Proof: We will construct $\mathcal{S}$ based on the given (black-box) deterministic protocol $\mathcal{P}$. The constructed strategy $\mathcal{S}$ will determine the sequence of probes that the player should do in each round $r$, so that the probing outcomes will enable the player to fully simulate the execution of $\mathcal{P}$. During the course of the probing game, we say that a worker node $i$ has been hit if there has been some probe $(i, j)$ that is a hit. In any given round $r$ of $\mathcal{P}$ 's execution, we say that a group leader node $i$ sends an influential message in round $r$ if node $i$ sends (i.e., locally broadcasts) a message in round $r$ and the adversary does not fail the dummy node connecting node $i$ with the root at the beginning of round $r+1$. Note that since node $i$ is a group leader in round $r$, it is guaranteed (by design of our adversary) that the dummy node has not failed in round $r$ or earlier. If the adversary indeed fails that dummy node at the beginning of round $r+1$, then node $i$ will no longer be a group leader in round $r+1$. Furthermore, node $i$ (and node $i$ 's group) will be merged with another group, with another node being the new (merged) group's leader.

We will prove that when the player uses our constructed probing strategy $\mathcal{S}$, all the following properties hold for all round $r$ where $0 \leq r \leq R$. Here $R$ is the total number of rounds in $\mathcal{P}$ 's execution over $W$, which must be finite. Recall that round 1 is the first round where there can possibly be a message sent in $\mathcal{P}$ 's execution.

Property 1 In round $r$ of the probing game, if the player does a probe $(i, j)$ and if this probe is a hit, then in round $r$ of $\mathcal{P}$ 's execution, nodes $i$ 's group leader must send an influential message. 
Property 2 In $\mathcal{P}$ 's execution, if a group leader sends an influential message in round $r$, then all nodes in that group have been hit by the player in the probing game by the end of round $r$.

Property 3 In $\mathcal{P}$ 's execution, immediately after the adversary injects potential failures at the beginning of round $r+1$, in each group there is at most one worker node that has not been hit by the player in the probing game. ${ }^{14}$

Property 4 In round $r$, the player in the probing game can generate all influential messages sent by all group leaders in round $r$ of $\mathcal{P}$ 's execution.

Lemma 43 below proves that the above 4 properties indeed hold under a properly constructed probing strategy $\mathcal{S}$. Now by Property 4, since the influential messages from group leaders are the only messages that can affect the root via the dummy nodes, the player will be able to simulate those dummy nodes and all incoming messages to the root throughout the execution. Then the root will be able to produce a final result in round $R$, and the player simply uses this result as the result to the probing game. Next if the total number of hits in the probing game reaches $n$, then all nodes must have been hit since each node can only contribute one hit. By Property 3, we know that in any round, each group can have at most one worker node that has not been hit. Initially there are $n$ groups, and thus there must exist at least $\frac{n}{2}$ groups where each group contributes a hit. By Property 1, the $\frac{n}{2}$ leaders of these $\frac{n}{2}$ groups will each send an influential message. Once all these influential messages are sent, our adversary will introduce failures so that there will be $\frac{n}{2}$ new groups, with these $\frac{n}{2}$ nodes as new leaders. Since we still need to have $\frac{n}{2}$ more hits and since each group can contribute at most one hit, among those $\frac{n}{2}$ groups, there must exist $\frac{n}{4}$ groups whose leaders will each send a second influential message. Continuing such argument will show that in order for the total number of hits in the probing game to reach $n$, some node in the SUM protocol $\mathcal{P}$ will have to send at least $a$ influential messages, where $a$ is the total number of terms in the summation of $n=\frac{n}{2}+\frac{n}{4}+\ldots+2+1+1$. Observing that $a=\log _{2} n+1$ and that $a$ messages translate to at least $a$ bits completes the proof.

Lemma 43 Under the conditions of Theorem 42, there exists a probing strategy $\mathcal{S}$ such that the 4 properties described in the proof of Theorem 42 hold for all round $r$ where $0 \leq r \leq R$. Here $R$ is the total number of rounds in $\mathcal{P}$ 's execution over $W$.

Proof: We prove via an induction on $r$. For $r=0$, we construct $\mathcal{S}$ such that no probes are done in round 0 . The 4 properties trivially hold for round 0 . Now consider any round $r>0$, while assuming that they hold for all rounds before $r$.

We first construct the set of probes that the player should do in round $r$. Consider any group $g$ in round $r$ of $\mathcal{P}$ 's execution, after the adversary injects potential failures at the beginning of round $r$. (Note that failures affect group membership, and thus we explicitly state that $g$ is defined after the failures have been injected in round $r$.) By inductive hypothesis on Property 3, there is at most one worker node miss $(g)$ in $g$ that has not been hit. Thus the player knows the value of all other nodes in $g$. If miss $(g)$ exists, then the player will exhaustively enumerate all $j$ 's such that there has not been a probe $(\operatorname{miss}(g), j)$. Intuitively, $j$ has not been ruled out as a possible value for miss $(g)$. For each such $j$, the player tries simulating $\mathcal{P}$ 's execution on all nodes in $g$, from round 0 to round $r$ (inclusive). Doing so will enable the player to determine the message (if any) sent in round $r$ by $g$ 's group leader. Such simulation is possible since the player has the values of all the nodes in that group, and also because by inductive hypothesis on Property 4, the player can generate all influential messages sent by other group leaders up to round $r-1$. In particular for a node $i$ in $g$, all incoming messages from nodes outside of $g$ must be sent from those dummy nodes connecting node $i$ with those group leaders in round 1 through round $r-1$. The reason is that in any round from 1 through $r-1$, non-leader

\footnotetext{
${ }^{14}$ First, we explicitly mention "after failure injection" since group membership is affected by failures. Second, we consider the beginning of round $r+1$ instead of the beginning of round $r$ to facilitate our later proof by induction.
} 
group members can only have neighboring dummy nodes connecting them to other nodes in their own groups and not to node $i$. Furthermore, non-influential messages from a group leader can never affect either the root or nodes in other groups (via the corresponding dummy nodes), since those dummy nodes will be failed right after they receive those non-influential messages. Finally, we do not yet know what influential messages other group leaders will send in round $r$, but those will not affect the potential message sent in round $r$ by $g$ 's group leader.

We say that a $(\operatorname{miss}(g), j)$ combination is a candidate probe if miss $(g)$ exists and by the above process, the player has determined that there will be a message sent in round $r$ by $g$ 's group leader if $j$ is the value of node miss $(g)$. (We do not yet know whether this message will be influential.) Next the player orders all candidate probes, using $g$ as the primary key and $j$ as the secondary key. In round $r$, the player sequentially issues the probes in this ordered list, subject to the following two constraints. First, if a certain $(\operatorname{miss}(g), j)$ probe is a hit, then the player will skip all following probes in the form of $\left(\operatorname{miss}(g), j^{\prime}\right)$ for all $j^{\prime}$. Second, if the number of hits so far is such that the adversary is ready to inject the next batch of failures, the player skips all the remaining probes in the ordered list.

We next prove that the probing strategy constructed as above does satisfy the 4 properties. Property 1 clearly holds since a hit of $(\operatorname{miss}(g), j)$ means that node $\operatorname{miss}(g)$ indeed has a value of $j$. Given the trial simulation and since everything is deterministic, miss $(g)$ 's group leader will send a message in round $r$ of $\mathcal{P}$ 's execution. Furthermore, since the probes are done sequentially and since the player must have encountered this hit before the adversary is ready to inject the next batch of failures, the adversary will not fail the dummy node connecting miss $(g)$ 's group leader to the root at the beginning of round $r+1$.

For Property 2, we need to prove that if a group $g$ 's group leader sends an influential message in round $r$, then all nodes in $g$ have been hit by the end of round $r$. If miss $(g)$ does not exist, we already hit all nodes in $g$. If miss $(g)$ exists, let $j$ be the value of the node miss $(g)$. Clearly, there has never been a probe $(m i s s(g), j)$. By our construction of the probing strategy in round $r,(\operatorname{miss}(g), j)$ will be a candidate probe. If the player indeed probes $(\operatorname{miss}(g), j)$ in round $r$, then miss $(g)$ will be hit in round $r$ and we are done. If the player does not probe $(m i s s(g), j)$ in round $r$, the only possibility is that the adversary is ready to inject the next batch of failures at the beginning of round $r+1$. In such a case, the adversary will fail the dummy node connecting $g$ 's group leader to the root, making the message (if any) sent by $g$ 's group leader non-influential.

For Property 3, if the adversary does not inject failures at the beginning of round $r+1$, then clearly the property inherited from the beginning of round $r$ continues to hold at the beginning of round $r+1$. If the adversary does inject failures at the beginning of round $r+1$, then the group membership in round $r$ and the group membership in round $r+1$ are different. Let $g_{1}, g_{2}, \ldots, g_{l}$ be the $l$ groups in round $r$, immediately after the adversary potentially inject failures at the beginning of round $r$. Without loss of generality, assume that after the failures are injected, $g_{i+l / 2}$ is merged with $g_{i}$ (for $1 \leq i \leq l / 2$ ) to form a new group, with $g_{i}$ 's leader being the leader of the new group. By inductive hypothesis on Property 3 , immediately after the adversary potentially injects failures at the beginning of round $r, g_{i+l / 2}(1 \leq i \leq l / 2)$ has at most one node that has not been hit. Given how the adversary injects failures, we know that the leader of $g_{i}(1 \leq i \leq l / 2)$ must have sent an (influential) message in round $r$ or earlier and after group $g_{i}$ is formed. By Property 2 (both in round $r$ and in earlier rounds), we know that all nodes in group $g_{i}$ have been hit by the end of round $r$. This means that after merging $g_{i}$ and $g_{i+l / 2}$, the new group still only has at most one node that has not been hit.

Finally for Property 4, consider any given group $g$ whose leader sends an influential message in round $r$. By Property 2, we know that all nodes in $g$ have been hit by the end of round $r$, and thus the player knows all their values. Same as in the earlier trial simulation, by inductive hypothesis on Property 4, the player can generate all influential messages sent by other group leaders up to and including round $r-1$. This means that the player can generate all incoming messages (up to and including round $r-1$ ) that may affect nodes in $g$. By same arguments as earlier, the player will be able to simulate $\mathcal{P}$ 's execution on all nodes in $g$ from round 0 to round $r$ (inclusive). Also note that the messages received in round $r$, which we do not know yet, will not affect the messages sent by $g$ 's group leader in round $r$. Thus the player can generate that influential message 
sent by the group leader in round $r$.

\section{G.3 Lower Bound on the Number of Hits in the Probing Game}

With the strong connection between SUM and the probing game proved in the previous section, we now only need to obtain a lower bound on the probing game. A simpler version of this probing game was analyzed in [14] to reason about silence-based communication, in a failure-free setting. There the player is not allowed to interleave probes on $w_{i}$ with probes on $w_{i^{\prime}}$. Thus for our purpose, we prove the following lower bound result on the probing game where the probes may be arbitrarily interleaved:

Lemma 44 Consider the set $U$ of all the $(3 n)^{n}$ possible inputs to the probing game $(n \geq 2)$ and any given (adaptive) deterministic probing strategy that can give correct (zero-error) results for at least $\frac{2}{3}$ fraction of those inputs. There must exist an input such that using that strategy, the player encounters $n$ hits under that input.

Proof: We prove by contradiction, and assume that there exists a deterministic probing strategy $\mathcal{S}$ that gives correct results for at least $\frac{2}{3}$ fraction of inputs and has at most $n-1$ hits for all inputs.

Consider any given input $W=\left(w_{1}, w_{2}, \ldots, w_{n}\right) \in U$, which is initially unknown to the player. At any point of time during the game, we define $w_{i}$ 's residual domain (denoted as $D_{i}$ ) to be the set $\{j\}$ if there has been a probe $(i, j)$ so far which is a hit. Otherwise $w_{i}$ 's residual domain $D_{i}$ is defined to be the set:

$$
\{0,1,2, \ldots, 3 n-1\} \backslash\{j \mid \text { there has been a probe }(i, j)\}
$$

Intuitively, $w_{i}$ 's residual domain is the possible domain of $w_{i}$ given the probe outcomes so far. When the game ends under $\mathcal{S}, W$ has a unique residual domain vector $D=\left(D_{1}, D_{2}, \ldots, D_{n}\right)$, where $D_{i}$ is the residual domain of $w_{i}$. We next prove a simple useful property on $W$ 's residual domain vector to facilitate later reasoning. We claim that for any input $Z=\left(z_{1}, z_{2}, \ldots, z_{n}\right)$ where $z_{i} \in D_{i}$, the probes done by the player and all the probe outcomes must be identical under input $W$ and input $Z$. This in turn implies that $Z$ will have the same residual domain vector as well as the same final output as that of $W$. We prove this claim for the $k$ th probe, via a simple induction on $k$. The induction base for the zeroth probe clearly holds. Now consider the $k$ th probe. We already know that all previous probes and their outcomes are identical under $W$ and under $Z$. Since the player is deterministic, we know that the $k$ th probe will be the same under $W$ and $Z$. Let this probe be $(i, j)$. If $(i, j)$ is a hit for $W$, then we must have $D_{i}=\{j\}$. Since $z_{i} \in D_{i}$, we know that $z_{i}=j$ and the probe $(i, j)$ will be a hit for $Z$ as well. If $(i, j)$ is a miss for $W$, then we must have $j \notin D_{i}$. Since $z_{i} \in D_{i}$, we know that $z_{i} \neq j$ and thus the probe will be a miss for $Z$ as well. Thus the outcome of the $k$ th probe will be identical under input $W$ and input $Z$.

We now leverage the above property to prove the following claim. Define $U^{\prime}$ to be that set of inputs such that for each input $W \in U^{\prime}$, when the game ends, in $W$ 's residual domain vector $D=\left(D_{1}, D_{2}, \ldots, D_{n}\right)$ there exists some $D_{i}$ where $\left|D_{i}\right| \geq 2$. We claim that in order for the player to generate results correctly for at least $\frac{2}{3}$ fraction of all the inputs, $\left|U^{\prime}\right|$ must be no larger than $\frac{2}{3}|U|$.

We prove the above claim by contradiction and assume that $\left|U^{\prime}\right|>\frac{2}{3}|U|$. We partition $U^{\prime}$ into disjoint subsets such that all inputs in the same subset have the same residual domain vector. Consider any such subset $U_{D}^{\prime}$ where all inputs in the set has the same $D=\left(D_{1}, D_{2}, \ldots, D_{n}\right)$ as their residual domain vectors. By the earlier property on residual domain vector, we know that $U_{D}^{\prime}$ contains at least all those inputs $Z$ where $z_{i} \in D_{i}$ for $1 \leq i \leq n$, and all such inputs $Z$ will result in the same output. Furthermore, if an input $Z^{\prime}$ is such that $z_{i}^{\prime} \notin D_{i}$ for some $i$, then it is impossible for $Z^{\prime}$ to be in $U_{D}^{\prime}$. In other words, $U_{D}^{\prime}$ must be exactly the set of those inputs $Z$ where $z_{i} \in D_{i}$ for $1 \leq i \leq n$. Next without loss of generality, assume that $\left|D_{1}\right| \geq 2$. Consider any given $j_{2} \in D_{2}, j_{3} \in D_{3}, \ldots, j_{n} \in D_{n}$. For each $j \in D_{1}, Z=\left(j, j_{2}, j_{3}, \ldots, j_{n}\right)$ must be in $U_{D}^{\prime}$, and the player will produce the same output. Such output can be correct for at most one $j$. This in turn means 
that the player can generate a correct result for at most $\frac{1}{2}$ fraction of the inputs in $U_{D}^{\prime}$. Therefore for all the inputs in the set $U^{\prime}$, the player can generate correct results for at most $\frac{1}{2}$ fraction as well. Thus the player can generate a result correctly for at most $\frac{1}{2}\left|U^{\prime}\right|+\left|U \backslash U^{\prime}\right|=\frac{1}{2}\left|U^{\prime}\right|+|U|-\left|U^{\prime}\right|=|U|-\frac{1}{2}\left|U^{\prime}\right|<\frac{2}{3}|U|$ inputs.

We have just proved that $\left|U \backslash U^{\prime}\right| \geq \frac{1}{3}|U|$. We next use this result to prove that under some input in $U \backslash U^{\prime}$, the number of hits will be $n$. For every input $W \in U \backslash U^{\prime}$, we know that $W$ 's residual domain vector $D=\left(D_{1}, D_{2}, \ldots, D_{n}\right)$ satisfies $\left|D_{i}\right|=1$ for all $i$. Essentially, the player has "pinpointed" the value of each $w_{i}$ and knows $W$ precisely, instead of only its sum. This means that in the probing game, the player actually needs to learn the input precisely for at least $\frac{1}{3}$ fraction of all the inputs. We next prove that for the player to achieve such a goal, there will be $n$ hits on at least one of the inputs in $U \backslash U^{\prime}$.

Given such a goal, consider any given point of time where the player decides to do a probe $(i, j)$. (This necessarily means that there has not been a hit on $w_{i}$, and also means that $j \in D_{i}$ where $D_{i}$ is current residual domain of $w_{i}$.) Since the goal is to learn the input precisely, doing such a probe is no different from doing any other probe $\left(i, j^{\prime}\right)$ as long as $j^{\prime} \in D_{i}$. With such an observation, we can now make the following without loss of generality assumption: For any given input $W$ and any given $i$, consider all probes done by the player in the form of $\left(i, j_{0}\right),\left(i, j_{1}\right),\left(i, j_{2}\right), \ldots$ Without loss of generality, we can assume that $j_{0}=0, j_{1}=1, j_{2}=2$, $\ldots$ We know that under the probing strategy $\mathcal{S}$, there are at most $n-1$ hits under all inputs, including all inputs $W \in U \backslash U^{\prime}$. Consider any given $W \in U \backslash U^{\prime}$ and let $i$ be the index of the component that has not been hit. Since $\left|D_{i}\right|=1$ and by our earlier without loss of generality assumption, we know that $w_{i}=3 n-1$. However, the number of such inputs is:

$$
\mid\left\{W \mid W \in U \text { and } \exists i \text { such that } w_{i}=3 n-1\right\} \mid=(3 n)^{n}-(3 n-1)^{n}<\frac{1}{3}(3 n)^{n}, \text { for } n \geq 2 .
$$

This contradicts with $\left|U \backslash U^{\prime}\right| \geq \frac{1}{3}|U|$. Thus under some $W \in U \backslash U^{\prime}$, there will be $n$ hits.

\section{G.4 Proof for Theorem 6 in Section 7}

Now we are ready to prove Theorem 6 in Section 7. The following lemma is proved by first establishing a connection between randomized complexity and distributional complexity via well-known techniques [24], and then using Theorem 42 and Lemma 44 to obtain a lower bound on the distributional complexity. Recall from Appendix A that the notation $\mathcal{R}_{0, \delta}^{\text {syn,ft }}$ simply means $\mathcal{R}_{\epsilon, \delta}^{\text {syn,ft }}$ with $\epsilon=0$.

Lemma 45 Consider any $b \geq 1$ and any integer $N=\frac{(n+1)(n+2)}{2}$ where $n$ is a power of 2 . If in the SUM problem each node may take an integer value in $\{0,1, \ldots, 3 n-1\}$, then there exists a connected topology $G$ with $N$ nodes, such that:

$$
\mathcal{R}_{0, \frac{1}{3}}^{\mathrm{syn}, \mathrm{ft}}(\mathrm{SuM}, G, b) \geq \log n+1
$$

Proof: We let $G$ be the topology constructed in Appendix G.1, and consider the deterministic and adaptive adversary described there. It will be convenient to view this adversary as part of the Sum protocol. Namely, given any randomized SUM protocol, we can consider a randomized "augmented protocol" which repeatedly executes one round of the randomized Sum protocol and then invokes the (deterministic) adversary to potentially inject failures. We thus no longer need to discuss the adversary separately.

Now consider the optimal randomized augmented protocol that generates a zero-error result with probability at least $\frac{2}{3}$ for all input $W$, while incurring a worst-case (over the coin flips) communication complexity of $\mathcal{R}_{0, \frac{1}{3}}^{\text {syn,ft }}$ (SUM, $\left.G, b\right)$. If we subject this protocol against an input chosen uniformly randomly out of all possible inputs, then trivially the protocol still generates a zero-error result with probability at least $\frac{2}{3}$, where the probability is taken over both the input distribution and the random coin flips. Now let us view this 
randomized augmented protocol as a distribution over deterministic augmented protocols. Then there must exist at least one deterministic protocol $\mathcal{P}$ which can generate a zero-error result with probability at least $\frac{2}{3}$ under this uniform input distribution, since otherwise the expectation taken over all deterministic augmented protocols cannot reach $\frac{2}{3}$. Finally, let $a$ denote the maximum number of bits sent by a node, across all nodes when we run $\mathcal{P}$ against the worst-case input (that maximizes $a$ ). Since $\mathcal{P}$ is selected by the randomized algorithm with positive probability and since $\mathcal{R}_{0, \frac{1}{3}}^{\mathrm{Syn}, \mathrm{ft}}$ (SUM, $\left.G, b\right)$ is defined over worst-case coin flips, we have $a \leq \mathcal{R}_{0, \frac{1}{3}}^{\mathrm{syn}, \mathrm{ft}}(\mathrm{Sum}, G, b)$.

On the other hand, Theorem 42 tells us that given such a $\mathcal{P}$, there exists a corresponding probing strategy $\mathcal{S}$ in the probing game so that using $\mathcal{S}$, the player in the probing game can generate the same result as $\mathcal{P}$. Since $\mathcal{P}$ generates a zero-error result for at least $\frac{2}{3}$ fraction of the inputs, we know that the player using $\mathcal{S}$ generates a zero-error result for so many inputs as well. Next by Lemma 44, there exists some input $W$ such that the player encounters $n$ hits. In turn, Theorem 42 now tells us that when running the SUM protocol $\mathcal{P}$ against this input $W$, some node sends at least $\log n+1$ bits. Thus we have $\mathcal{R}_{0, \frac{1}{3}}^{\mathrm{syn}, \mathrm{ft}}(\operatorname{SUM}, G, b) \geq a \geq \log n+1$.

The following corollary extends the above lemma to our standard setting where nodes only have binary values.

Corollary 46 Consider any $b \geq 1$ and any integer $N \geq 5$. Let $n$ be the largest integer that is a power of 2 and satisfies $\frac{(n+1)(n+2)}{2}+n(3 n-1) \leq N$. There exists a connected topology $G$ with $N$ nodes, such that:

$$
\mathcal{R}_{0, \frac{1}{3}}^{\mathrm{syn}, \mathrm{ft}}(\mathrm{SuM}, G, b) \geq \log n+1
$$

Proof: Let $N_{1}=\frac{(n+1)(n+2)}{2}$ and we first construct a connected topology $G_{1}$ with $N_{1}$ nodes as described in Appendix G.1. Next we attach $(3 n-1)$ degree- 1 follower nodes to each work node in $G_{1}$, and attach $\left(N-N_{1}-n(3 n-1)\right)$ degree-1 nodes to the root of $G_{1}$. All those degree-1 nodes attached to $G_{1}$ 's root will always have value 0 . Let $G$ be the resulting $N$-node connected topology. One can trivially obtain a reduction from the Sum problem on $G_{1}$ (where each worker node has an integer value in $\{0,1, \ldots, 3 n-1\}$ ) to the Sum problem on $G$ (where each node has a binary value). In particular in the reduction, the root in $G_{1}$ will simulate the root in $G$ and also all the degree-1 neighbors of the root in $G$. Each worker node in $G_{1}$ will simulate the corresponding worker node and its $(3 n-1)$ followers in $G$. If the worker node $i$ in $G_{1}$ has a value of $w_{i}$, then in $G$ the first $w_{i}$ of the corresponding work node's follower nodes will have value 1 and the remaining $\left(3 n-1-w_{i}\right)$ follower nodes will have value 0 . Combining this reduction with the lower bound on the SUM problem on $G_{1}$ from Lemma 45 , we have $\mathcal{R}_{0, \frac{1}{3}}^{\mathrm{syn}, \mathrm{ft}}(\mathrm{SUM}, G, b) \geq \log n+1$.

The next corollary extends the above corollary to $\mathcal{R}_{\epsilon, \frac{1}{3}}^{\mathrm{syn}, \mathrm{ft}}$ for $\epsilon \geq \frac{1}{N}$.

Corollary 47 Consider any $b \geq 1$, any integer $N \geq 15$, and any $\epsilon \in\left[\frac{1}{N}, \frac{1}{15}\right]$. Let $n$ be the largest integer that is a power of 2 and satisfies $\frac{(n+1)(n+2)}{2}+n(3 n-1) \leq \frac{1}{3 \epsilon}$. There exists a connected topology $G$ with $N$ nodes, such that:

$$
\mathcal{R}_{\epsilon, \frac{1}{3}}^{\mathrm{syn}, \mathrm{ft}}(\mathrm{SUM}, G, b) \geq \log n+1
$$

Proof: Let $N_{1}=\frac{1}{3 \epsilon}$ and we first construct a connected topology $G_{1}$ with $N_{1}$ nodes such that $\mathcal{R}_{0, \frac{1}{3}}^{\text {syn,ft }}\left(\operatorname{Sum}, G_{1}, b_{1}\right) \geq \log n+1$ for any $b_{1}$. Corollary 46 ensures that such $G_{1}$ exists. Next we attach $N_{2}=N-N_{1}$ nodes to the root of $G_{1}$, and let the resulting topology be $G$. Those $N_{2}$ nodes will always have a value of 0 and will never fail. Note that the final sum on $G$ can never be above $\frac{1}{3 \epsilon}$. If we have at most $\epsilon$ relative error on the final sum on $G$, then the absolute error is at most $\frac{1}{3 \epsilon} \cdot \epsilon=\frac{1}{3}$. Since the exact sum must 
be an integer, to generate a result with $\epsilon$ relative error in $G$, the protocol intuitively needs to produce a zeroerror result. Putting it another way, if the output is not an integer, we can always output the closest integer instead. Doing so will never cause an output that was previously within $\epsilon$-error bound to exceed the $\epsilon$-error bound after the conversion. The above observation enables a trivial reduction from $\mathcal{R}_{0, \frac{1}{3}}^{\mathrm{syn}, \mathrm{ft}}$ (SUM, $\left.G_{1}, b_{1}\right)$ to $\mathcal{R}_{\epsilon, \frac{1}{3}}^{\mathrm{syn}, \mathrm{ft}}(\mathrm{SuM}, G, b)$, which gives:

$$
\mathcal{R}_{\epsilon, \frac{1}{3}}^{\mathrm{syn}, \mathrm{ft}}(\operatorname{SuM}, G, b) \geq \mathcal{R}_{0, \frac{1}{3}}^{\mathrm{syn}, \mathrm{ft}}\left(\mathrm{SUM}, G_{1}, b_{1}\right) \geq \log n+1
$$

Proof for Theorem 6: We first prove the lower bound on $\mathcal{R}_{0}^{\mathrm{syn}, \mathrm{ft}}\left(\mathrm{SuM}_{N}, b\right)$. For any $N \geq 5$, consider the $N$-node connected topology $G$ as constructed by Corollary 46. Together with Lemma 7, we trivially have:

$$
\mathcal{R}_{0}^{\mathrm{syn}, \mathrm{ft}}\left(\operatorname{SuM}_{N}, b\right) \geq \frac{1}{3} \mathcal{R}_{0, \frac{1}{3}}^{\mathrm{syn}, \mathrm{ft}}\left(\operatorname{SuM}_{N}, b\right) \geq \frac{1}{3} \mathcal{R}_{0, \frac{1}{3}}^{\mathrm{syn}, \mathrm{ft}}(\mathrm{SUM}, G, b) \geq \frac{1}{3}(\log n+1)
$$

By Corollary 46, here $n$ is the largest integer that is a power of 2 and satisfies $\frac{(n+1)(n+2)}{2}+n(3 n-1) \leq N$. Thus we have $n=\Theta(\sqrt{N})$, which implies $\mathcal{R}_{0}^{\text {syn,ft }}\left(\operatorname{SuM}_{N}, b\right)=\Omega(\log N)$.

We next prove the lower bound on $\mathcal{R}_{\epsilon, \frac{1}{3}}^{\mathrm{syn}, \mathrm{ft}}\left(\operatorname{SuM}_{N}, b\right)$ for $\epsilon \geq \frac{1}{N}$. For any $N \geq 15$, consider the $N$-node connected topology $G$ as constructed by Corollary 47 . We trivially have:

$$
\mathcal{R}_{\epsilon, \frac{1}{3}}^{\mathrm{syn}, \mathrm{ft}}\left(\mathrm{SuM}_{N}, b\right) \geq \mathcal{R}_{\epsilon, \frac{1}{3}}^{\mathrm{syn}, \mathrm{ft}}(\mathrm{SUM}, G, b) \geq \log n+1
$$

By Corollary 47, here $n$ is the largest integer that is a power of 2 and satisfies $\frac{(n+1)(n+2)}{2}+n(3 n-1) \leq \frac{1}{3 \epsilon}$. Thus we have $n=\Theta\left(\frac{1}{\sqrt{\epsilon}}\right)$, which implies $\mathcal{R}_{\epsilon, \frac{1}{3}}^{\mathrm{syn}, \mathrm{ft}}\left(\mathrm{SuM}_{N}, b\right)=\Omega\left(\log \frac{1}{\epsilon}\right)$.

Finally, for $\epsilon=\Omega\left(\frac{1}{N}\right)$ but $\epsilon<\frac{1}{N}$ (in which case $\epsilon$ is necessarily $\Theta\left(\frac{1}{N}\right)$ ), we have:

$$
\mathcal{R}_{\epsilon, \frac{1}{3}}^{\mathrm{syn}, \mathrm{ft}}\left(\operatorname{SuM}_{N}, b\right) \geq \mathcal{R}_{\frac{1}{N}, \frac{1}{3}}^{\mathrm{syn}}\left(\operatorname{SuM}_{N}, b\right)=\Omega(\log N)=\Omega\left(\log \frac{1}{\epsilon}\right)
$$

\section{H Trivially Extending Our Results to Alternative Models}

\section{H.1 Excluding The Communication Complexity of The Root Node}

Our model in Section 2 defined the communication complexity of a SUM protocol to be the number of bits sent by the bottleneck node. Here it is possible for the bottleneck node to be the root. In some scenarios, one may want to exclude the root in this definition. Excluding the root clearly makes the NFT upper bounds easier to achieve, so all our upper bounds trivially carry over. This section further shows that excluding the root does not affect any asymptotic FT lower bounds either. This is true for all FT lower bounds, and not just for FT lower bounds obtained in this paper.

Let $G_{1}$ be the topology for obtaining the FT lower bounds, when the root's communication complexity is included in the definition of communication complexity for the SUM protocol. We construct a new lower bound topology $G_{2}$ by attaching a new degree- 1 node to $G_{1}$ 's root, and let this new node be $G_{2}$ 's root. The following trivial reduction shows that the communication complexity (while excluding the root) on $G_{2}$ is at 
least as large as the communication complexity (while including the root) on $G_{1}$. Consider any Sum protocol $\mathcal{P}_{2}$ for $G_{2}$ whose communication complexity is $a$, when excluding the communication complexity of $G_{2}$ 's root. We construct a corresponding SuM protocol $\mathcal{P}_{1}$ for $G_{1}$, which is the same as $\mathcal{P}_{2}$ except that $G_{1}$ 's root additionally locally simulates the execution of $\mathcal{P}_{2}$ on $G_{2}$ 's root. Clearly, the communication complexity incurred by $G_{2}$ 's root will not contribute to the communication complexity of $\mathcal{P}_{1}$. As a result, the communication complexity of $\mathcal{P}_{1}$ is exactly $a$ as well, when including the communication complexity of $G_{1}$ 's root.

One still needs to take care of a minor technicality in the above reduction: An aggregation round in $\mathcal{P}_{2}$ 's execution over $G_{2}$ actually has one more round than an aggregation round in $\mathcal{P}_{1}$ 's execution over $G_{1}$. While the two executions in our above reduction take the exactly same number of rounds, they may take slightly different numbers of aggregation rounds. However, since an aggregation round usually has $\omega(1)$ rounds with respect to the size of $G_{1}$ (e.g., in all our constructions), this will not make any real difference when the size of $G_{1}$ is sufficiently large.

\section{H.2 Defining Time Complexity Using Average-Case Coin Flips}

Our model in Section 2 defined the time complexity of a randomized protocol as the number of rounds needed for the protocol to terminate, under the worst-case random coin flips in the protocol. This section shows that if we define the time complexity using average-case random coin flips, our exponential gap claim will continue to hold and our FT lower bounds on communication complexity will only be slightly affected. In particular, the FT lower bounds for zero-error results will now be obtained indirectly via the FT lower bounds for $(\epsilon, \delta)$ approximate results while taking the smallest $\epsilon$ possible. The quality of the FT lower bounds for zero-error results obtained in such a way remains high because our results for the $(\epsilon, \delta)$-error case are strong enough. The following provides a more detailed discussion.

Similar as in Section 2, we define $\mathcal{R}_{0}^{\mathrm{syn}, \mathrm{ft}, \mathrm{avg}}$ (SUM, $\left.G, b\right)$ and $\mathcal{R}_{\epsilon, \delta}^{\mathrm{syn}, \mathrm{ft}, \mathrm{avg}}$ (SUM, $\left.G, b\right)$ to be the smallest communication complexity of FT SUM protocols over the topology $G$, where the protocol terminates within $b$ aggregation rounds on expectation (with the expectation taken over the coin flips in the protocol). We then define $\mathcal{R}_{0}^{\text {syn,ft,avg }}\left(\operatorname{SuM}_{N}, b\right)$ and $\mathcal{R}_{\epsilon, \delta}^{\text {syn,ft,avg }}\left(\operatorname{SuM}_{N}, b\right)$ to be the maximum $\mathcal{R}_{0}^{\text {syn,ft,avg }}(\operatorname{SuM}, G, b)$ and $\mathcal{R}_{\epsilon, \delta}^{\text {syn,ft,avg }}$ (SUM, $\left.G, b\right)$, respectively, across all topology $G$ 's where $G$ is connected and has exactly $N$ nodes. The notation $\mathcal{R}_{0, \delta}^{\text {syn,ft,avg }}$ simply means $\mathcal{R}_{\epsilon, \delta}^{\text {syn,ft,avg }}$ with $\epsilon=0$. We also define the corresponding NFT versions of these definitions.

All the NFT upper bounds from Section 3 trivially hold under this new definition of time complexity, since the upper bound protocol is actually deterministic. The FT lower bounds of $\Omega(\log N)$ and $\Omega\left(\log \frac{1}{\epsilon}\right)$ for unrestricted $b$ (Section 7) obviously continue to hold as well, since those FT lower bounds do not depend on the time complexity of the protocol. We next show that for $b \leq N^{0.125-c}$ or $\frac{1}{\epsilon^{0.5-c}}$, similar lower bounds on $\mathcal{R}_{0}^{\text {syn,ft,avg }}$ and $\mathcal{R}_{\epsilon, \delta}^{\text {syn,ft,avg }}$ can be obtained as the lower bounds on $\mathcal{R}_{0}^{\text {syn,ft }}$ and $\mathcal{R}_{\epsilon, \delta}^{\text {syn,ft }}$ in Section 5 .

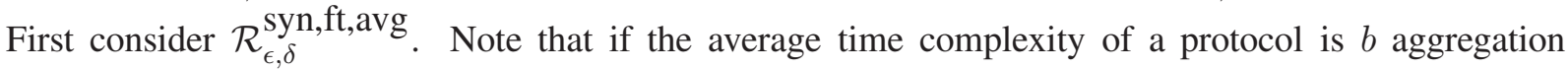
rounds, then by Markov's inequality, the time complexity of the protocol can be above $\frac{b}{\delta^{\prime}}$ aggregation rounds with probability at most $\delta^{\prime}$ (for any positive $\delta^{\prime}$ ). Recall our reduction (in Section 5 and Appendix D) from UNIONSIZECP $_{n, q}$ to SUM where the simulation can last $q n$ rounds. In that reduction we set $q=5 b$ so that $q n$ rounds are at least $b$ aggregation rounds. Now for obtaining the lower bound on $\mathcal{R}_{\epsilon, \delta}^{\mathrm{syn}, \mathrm{ft} \text {,avg }}$, all we need to do is to set $q=\frac{5 b}{\delta^{\prime}}$ instead, so that $q n$ rounds are at least $\frac{b}{\delta^{\prime}}$ aggregation rounds. If the SUM oracle protocol does not terminate within $q n$ rounds, which happends with at most $\delta^{\prime}$ probability, Alice simply outputs an 
arbitrary answer. Take $\delta^{\prime}=\frac{1}{30}$ and we have:

$$
\begin{aligned}
\mathcal{R}_{\epsilon, \frac{1}{6}}^{\text {syn,ft,avg }}\left(\operatorname{SuM}_{N}, b\right) & \geq \frac{1}{2} \mathcal{R}_{\epsilon, \frac{1}{6}+\frac{1}{30}}^{\text {syn }}\left(\operatorname{UNIONSIZECP}_{n, q}, q n\right) \\
& \geq \frac{1}{2} \mathcal{R}_{\epsilon, \frac{1}{5}}^{\mathrm{syn}}\left(\mathrm{UNIONSIZECP}_{n, q}, 150 b n\right) \\
& =\Omega\left(\frac{1}{\epsilon b^{2} \log N}\right), \text { for } \epsilon=\Omega\left(\frac{1}{\sqrt[4]{N}}\right)
\end{aligned}
$$

Essentially, this means that for $b \leq \frac{1}{\epsilon^{0.5-c}}$, the lower bound on $\mathcal{R}_{\epsilon, \frac{1}{6}}^{\mathrm{syn}, \mathrm{ft} \text {,avg }}$ is asymptotically the same as the lower bound on $\mathcal{R}_{\epsilon, \frac{1}{5}}^{\mathrm{syn}, \mathrm{ft}}$.

We next move on to the lower bound for $\mathcal{R}_{0}^{\text {syn,ft,avg }}$ when $b \leq N^{0.125-c}$. It is not possible to use the above trick anymore since here the result has to be zero-error. However, by a similar argument as in Lemma 7 , we have for any $b \geq 1$ and $\delta>0$ :

$$
\mathcal{R}_{0}^{\mathrm{syn}, \mathrm{ft}, \mathrm{avg}}\left(\mathrm{SuM}_{N}, b\right) \geq \delta \mathcal{R}_{0, \delta}^{\mathrm{syn}, \mathrm{ft}, \mathrm{avg}}\left(\mathrm{SuM}_{N}, b\right)
$$

Thus we have:

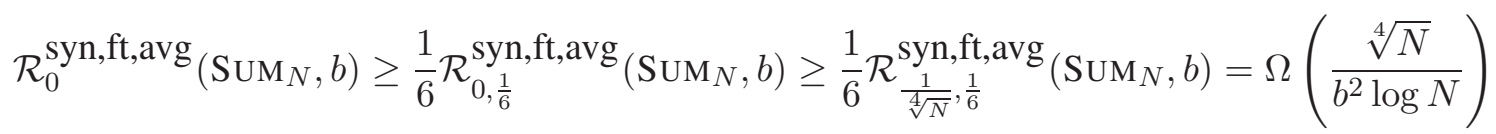

Compared to the lower bound on $\mathcal{R}_{0}^{\text {syn,ft }}$, we have a weaker term of $\sqrt[4]{N}$ here instead of $\sqrt{N}$. Nevertheless, the exponential gap continues to exist. 